\title{
MICRO-NOZZLE SIMULATION AND TEST FOR AN ELECTROTHERMAL PLASMA THRUSTER
}

\author{
A Thesis \\ presented to \\ the Faculty of California Polytechnic State University, \\ San Luis Obispo
}

\author{
In Partial Fulfillment \\ of the Requirements for the Degree \\ Master of Science in Aerospace Engineering
}

by

Tyler Croteau

December 2018 
(c) 2018

Tyler Croteau

ALL RIGHTS RESERVED 


\section{COMMITTEE MEMBERSHIP}

TITLE:

Micro-Nozzle Simulation and Test for an Electrothermal Plasma Thruster

AUTHOR:

Tyler Croteau

DATE SUBMITTED: December 2018

COMMITTEE CHAIR: Amelia Greig, Ph.D.

Professor of Aerospace Engineering

COMMITTEE MEMBER: Dianne DeTurris, Ph.D.

Professor of Aerospace Engineering

COMMITTEE MEMBER: Graham Doig, Ph.D.

Professor of Aerospace Engineering

COMMITTEE MEMBER: Roderick Boswell, Ph.D.

Professor in the Research School of Physics

and Engineering, Australian National University 


\begin{abstract}
Micro-Nozzle Simulation and Test for an Electrothermal Plasma Thruster

Tyler Croteau
\end{abstract}

With an increased demand in Cube Satellite (CubeSat) development for low cost science and exploration missions, a push for the development of micro-propulsion technology has emerged, which seeks to increase CubeSat capabilities for novel mission concepts. One type of micro-propulsion system currently under development, known as Pocket Rocket, is an electrothermal micro-thruster. Pocket Rocket uses a capacitively coupled plasma generated by radio-frequency $(\mathrm{RF})$, in order to provide neutral gas heating via ion-neutral collisions within a gas discharge tube. When compared to a cold-gas thruster of similar size, this gas heating mechanism allows Pocket Rocket to increase the exit thermal velocity of its gaseous propellant for increased thrust, upon expansion into the vacuum of space. Previous experimental work has only investigated use of the gas discharge tube's orifice for propellant expansion. This thesis aims to answer if Pocket Rocket may see an increase in thrust with the addition of a micro-nozzle placed at the end of the gas discharge tube.

Computational Fluid Dynamics (CFD) simulations are performed in the software package CFD-ACE+. The simulation cases consist of varying micro-nozzle conical geometries (half angles $\alpha=15^{\circ}-60^{\circ}$ and area ratios $\epsilon=5-100$ ) in order to characterize a design that best minimizes viscous losses in the throat and diverging section of the nozzle. The conical nozzle design with $\alpha=30^{\circ}$ and $\epsilon=10$ is manufactured and tested in order to validate the CFD model. A pitot-static tube and two convectron gauges are used to characterize the exhaust plume of Pocket Rocket by measuring the total and static pressures at various locations within the flow. During micro-nozzle testing, this instrumentation was able to directly characterize oblique shock structures in the exhaust plume. 
Simulation results indicate that the micro-nozzles with half angles of $30^{\circ}$ produce the highest performance increases. Geometries with $\alpha=15^{\circ}$ and $60^{\circ}$ limited the enhancement of performance due to both an increase in large viscous boundary layer formation within the nozzle divergent section, and significant flow detachment at the nozzle throat, respectively. Smaller area ratios of $\epsilon=10$ were also found to produce the largest increases in thrust. Overall, performance increases with the addition of a micro-nozzle for Pocket Rocket are found to be approximately $6 \%$ during plasma operation, and $25 \%$ during cold gas operation. This nozzle performance increase over the cold gas orifice configuration would be of significant value to Pocket Rocket in a contingency, loss of operational power scenario. With the addition of the best performing micro-nozzle, propellant heating is observed to increase by $60 \mathrm{~K}$. By placing a micro-nozzle at the end of Pocket Rocket's gas discharge tube, the propellant gas is no longer allowed to expand and cool within the plasma bulk region, thereby leading to increases in effective propellant heating. 


\section{ACKNOWLEDGMENTS}

Thanks to:

- Dr. Greig, for your incredible amount of time, expertise, and for guiding me through the world of electric propulsion.

- My thesis committee, for your invaluable time, feedback, and direct expertise during my time at Cal Poly.

- My mom, dad, and sister, for showing me what hard work and determination really looks like, and for always believing in me.

- Michaela, for spending your nights in the lab with me, your days encouraging me, and for your unrelenting support throughout this journey. 


\section{TABLE OF CONTENTS}

$\begin{array}{lll}\text { Page } & \end{array}$

LIST OF TABLES . . . . . . . . . . . . . . . . . . ix

LIST OF FIGURES . . . . . . . . . . . . . . . . . . . $\quad$ x

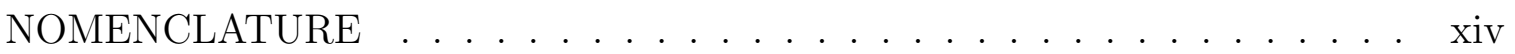

\section{CHAPTERS}

1 Introduction . . . . . . . . . . . . . . . . . . . . 1

1.1 Micro-Propulsion . . . . . . . . . . . . . . . . 2

1.1.1 Chemical Propulsion . . . . . . . . . . . . . . 4

1.1.2 Electric Propulsion . . . . . . . . . . . . . . . 5

1.2 The Pocket Rocket Thruster . . . . . . . . . . . . . . . 8

1.2.1 Plasma Theory . . . . . . . . . . . . . . . 8

1.2.2 Sheath Breakdown . . . . . . . . . . . . 10

1.2.3 Secondary Electrons . . . . . . . . . . . . . . . . . 11

1.2.4 Capacitively Coupled Plasma . . . . . . . . . . . . . . . 12

1.2.5 Thruster Operating Principles . . . . . . . . . . . . . . 12

1.2.6 Neutral Gas Heating in Pocket Rocket . . . . . . . . . . . . . 14

1.3 Basic Nozzle Theory . . . . . . . . . . . . . . . . . . . . . . 15

1.3.1 Nozzle Geometry . . . . . . . . . . . . . . . . . . . 16

1.4 Previous Studies on Micro-Nozzle Optimization . . . . . . . . . . . 18

1.5 Thesis Scope. . . . . . . . . . . . . . . . . . 20

2 Micro-Nozzle Design Methodology . . . . . . . . . . . . . . . . . . . . . 22

2.1 Design Parameters and Isentropic Flow Relationships . . . . . . . . . 22

2.1 .1 Mach Number . . . . . . . . . . . . . 22

2.1 .2 Isentropic Flow . . . . . . . . . . . . . . . . . . . 23

2.1.3 Coefficient of Thrust . . . . . . . . . . . . . . . . 24

2.2 Geometry Selection . . . . . . . . . . . . . . 26

3 Thruster Exhaust Flow Characterization . . . . . . . . . . . . . . . . 29

3.1 Thruster Hardware . . . . . . . . . . . . . . . . . . . . . . 29

3.1 .1 Nozzle Fabrication . . . . . . . . . . . . . . . . . . . 30 
3.2 Test $\operatorname{Setup} \ldots \ldots \ldots \ldots$

3.2 .1 Pitot-Static Tube Instrumentation . . . . . . . . . . . . . . 33

3.2 .2 Testing Procedure . . . . . . . . . . . . . . . . 36

3.3 Experiment Results . . . . . . . . . . . . . . . . . . . . . . . . . . 38

3.3.1 Orifice Thruster Configuration Testing . . . . . . . . . . . . 39

3.3.2 MN-5 Nozzle Configuration Testing . . . . . . . . . . . . . 42

3.3.3 Observed and Measured Nozzle Shock Diamonds . . . . . . . . 43

4 Computational Fluid Dynamics _ . . . . . . . . . . . . . . . 50

4.1 Software: CFD-ACE $+\ldots \ldots \ldots \ldots \ldots$

4.1 .1 CFD Validation Mesh _. . . . . . . . . . . . 55

4.1.2 Preliminary CFD Temperature and Pressure . . . . . . . 57

4.2 CFD Validation . . . . . . . . . . . . . . . . . . . . . . . 59

4.2 .1 Orifice Configuration . . . . . . . . . . . . . . . . . . . 59

$4.2 .2 \quad$ MN-5 Nozzle Configuration . . . . . . . . . . . . . . 60

4.3 Micro-Nozzle Design Simulation Results . . . . . . . . . . . . . 64

5 Conclusion . . . . . . . . . . . . . . . . . . . . 76

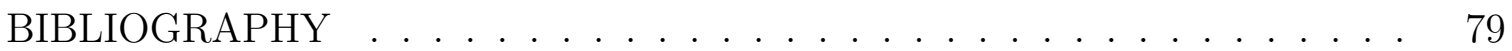
APPENDICES

A Convectron Calibration . . . . . . . . . . . . . 84 


\section{LIST OF TABLES}

Table

1.1 Range of performance values for micro-propulsion options with thrust $F$, specific impulse $I_{s p}$, and required power $P$. . . . . . . . . 4

1.2 Preliminary performance estimates for Pocket Rocket [1], with neutral gas temperature $\mathrm{T}_{g}$, mass flow rate $\dot{m}$, exit exhaust velocity $v_{e x}$, specific impulse $I_{s p}$, thrust $F$, plume power $P_{\text {plume }}$, and efficiency $\eta$.

2.1 Simulation cases for varying micro-nozzle conical geometries with diverging half angle $\alpha$, area ratio $\epsilon$, throat radius $R_{t}$, and nozzle cone length $L_{\text {cone }}$. . . . . . . . . . . . . . . . . . . . . .

4.1 Thruster housing thermal properties with density $\rho$, specific heat $c_{p}$, and thermal conductivity $k \ldots \ldots \ldots \ldots . \ldots$

4.2 Cold gas simulation performance results for mass flow rate $\dot{m}=3.3$ $\mathrm{mg} / \mathrm{s}$, ambient pressure $P_{a}=1 \mathrm{~Pa}$, plenum pressure $P_{p l}$, average exit exhaust velocity $v_{e x}$, thrust $F$, and specific impulse $I_{s p}$. Geometries vary with diverging half angle $\alpha$ and area ratio $\epsilon$. . . . . . .

4.3 Simulated plasma heating performance results for mass flow rate $\dot{m}=3.3 \mathrm{mg} / \mathrm{s}$, ambient pressure $P_{a}=1 \mathrm{~Pa}$, plenum pressure $P_{p l}$, neutral gas temperature $T_{g}$, average exit exhaust velocity $v_{e x}$, thrust $F$, and specific impulse $I_{s p}$. Geometries vary with diverging half angle $\alpha$ and area ratio $\epsilon$. . . . . . . . . . . . . . . . . 


\section{LIST OF FIGURES}

Figure

1.1 Paschen curve for argon gas. . . . . . . . . . . . . . . . 10

1.2 Generic sheath regions in a plasma with main plasma bulk potential $V_{p}$, presheath potential $V_{P s}$, and wall potential $V_{W a l l}$. . . . . . . 11

1.3 Pocket Rocket cross-section schematic. . . . . . . . . . . . . . . 13

1.4 Converging-Diverging nozzle concept. . . . . . . . . . . . 16

1.5 Conical nozzle geometry. . . . . . . . . . . . . . . . . 17

2.1 Nozzle area ratio vs. coefficient of thrust for argon gas. . . . . . . . 25

3.1 Schematic cross-section for modular thruster. . . . . . . . . . . 29

3.2 Modular Pocket Rocket components (from left to right); aluminum housing with plenum chamber barbed connections, aluminum grounding ring, SMA connector/Macor housing/alumina gas discharge tube, original thruster orifice end-plate, MN-5 nozzle end-plate. . . . . . .

3.3 (a) MN-5 machined nozzle close-up, (b) Nozzle to thruster installation with original orifice end-plate in the background, (c) Thruster

3.4 Experiment piping and instrumentation diagram. . . . . . . . . . 32

3.5 Experiment electrical equipment, vacuum chamber, rotary pump, and propellant feed system. . . . . . . . . . . . . 33

3.6 Pitot-static tube with convection gauges. . . . . . . . . . . . 35

3.7 Normal distribution probability plot with 95\% confidence intervals for cold gas MN-5 nozzle $\frac{P_{s}}{P_{0}}$ measurements at 0.05 inches from the thruster exit (blue circles) and 3 inches from the thruster exit (red squares). . . . . . . . . . . . . . . .

3.8 Orifice experimental results for (a) cold gas testing, and (b) plasma operation testing $\left(P_{p l}=2.9 \pm 0.5\right.$ Torr, $P_{a}=0.9 \pm 0.05$ Torr $)$. Vertical error bars represent $3 \sigma$, horizontal error bars represent 0.64 mm of parallax error. . . . . . . . . . . . . . . . . . .

3.9 Orifice thruster configuration testing with pitot tube at various locations within the thruster plume. 
3.10 MN-5 nozzle experimental results for (a) cold gas testing, and (b) plasma operation testing $\left(P_{p l}=6.1 \pm 0.5\right.$ Torr, $P_{a}=0.45 \pm 0.05$ Torr). Vertical error bars represent $3 \sigma$, horizontal error bars represent $0.64 \mathrm{~mm}$ of parallax error. . . . . . . . . . . . . . .

3.11 Zoomed in $\frac{P_{s}}{P_{0}}$ region of interest for MN-5 nozzle (a) cold gas testing and (b) plasma operation testing. Vertical error bars represent $3 \sigma$, horizontal error bars represent $0.64 \mathrm{~mm}$ of parallax error. . . . . . . 43

3.12 Approximate location of observed shock diamond during testing. . . 44

3.13 Nozzle test run with 3 observed shock diamonds. . . . . . . . . . . 45

3.14 Pitot-static tube bow shock. . . . . . . . . . . . . 46

3.15 Bow shock diagram. . . . . . . . . . . . . . . . 47

3.16 Mach number plot with 3 observed shock locations. . . . . . . . . 48

4.1 Full simulation domain for thruster with the orifice discharge tube geometry. . . . . . . . . . . . . . . 51

4.2 Full simulation domain for thruster with the MN-5 nozzle geometry. 52

4.3 Carpet plot of average power density within the gas discharge tube. 53

4.4 Ion current density within the gas discharge tube. . . . . . . . . . . 54

4.5 Full MN-5 nozzle simulation domain mesh. . . . . . . . . . . . 55

4.6 Mesh convergence results for velocity (blue squares), static pressure (red circles), and total pressure (red triangles). . . . . . . . . . . 57

4.7 Simulation temperature results for (top) orifice configuration at $P_{p l}=$ 3.4 Torr, $P_{a}=0.9$ Torr and (bottom) MN-5 nozzle configuration at $P_{p l}=6.6$ Torr, $P_{a}=0.45$ Torr. . . . . . . . . . . . . .

4.8 Simulation static pressure results for (left) orifice configuration at $P_{p l}=3.4$ Torr, $P_{a}=0.9$ Torr and (right) MN-5 nozzle configuration at $P_{p l}=3.4$ Torr, $P_{a}=0.9$ Torr. . . . . . . . . . . . . .

4.9 CFD validation results for the orifice thruster configuration during (a) cold gas operation and (b) plasma operation. The pink CFD (Average) results represent the simulation run at $P_{p l}=2.9$ Torr, $P_{a}=0.9$ Torr. The upper and lower CFD bounds were run with $P_{p l}=2.9 \pm 0.5$ Torr and $P_{a}=0.9 \pm 0.5$ Torr, respectively. . . . . .

4.10 Cold gas CFD validation results for the MN-5 nozzle thruster configuration, showing (a) full measurement profile and (b) zoomed in profile, just after the thruster exit. The upper and lower CFD bounds were run with $P_{p l}=6.1 \pm 0.5$ Torr and $P_{a}=0.45 \pm 0.5$ Torr, respec-

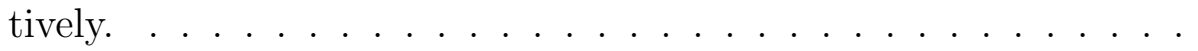


4.11 Plasma operation CFD validation results for the MN-5 nozzle thruster configuration, showing (a) full measurement profile and (b) zoomed in profile, just after the thruster exit. The upper and lower CFD bounds were run with $P_{p l}=6.1 \pm 0.5$ Torr and $P_{a}=0.45 \pm 0.5$ Torr, respectively. . . . . . . . . . . . . . . .

4.12 Zoomed in CFD static pressure profile through the MN-5 nozzle $\left(P_{p l}=6.6\right.$ Torr, $P_{a}=0.5$ Torr $)$. . . . . . . . . . . . . .

4.13 CFD Mach number plot showing downstream shock region after the MN-5 nozzle exit $\left(P_{p l}=6.6\right.$ Torr, $P_{a}=0.5$ Torr $)$. . . . . . . . .

4.14 Adapted CFD domain for the MN-1 through MN-9 nozzle geometry simulations. . . . . . . . . . . . . . . . 65

4.15 Flow detachment for the MN-8 nozzle. . . . . . . . . . . . . . 67

4.16 Simulated heating profile for (top) orifice thruster configuration and (bottom) MN-2 nozzle geometry. . . . . . . . . . . . . . . . . 68

4.17 Simulation pressure-distance values along an argon Paschen curve. . 70

4.18 Simulated heating nozzle velocity profile comparisons for (top) MN-2 geometry, (middle) MN-5 geometry, and (bottom) MN-8 geometry $\left(\dot{m}=3.3 \mathrm{mg} / \mathrm{s}, P_{a}=1 \mathrm{~Pa}\right) \ldots \ldots \ldots 71$

4.19 Simulated heating nozzle exit velocity plots for the MN-2, MN-5, and MN-8 geometries $\left(\dot{m}=3.3 \mathrm{mg} / \mathrm{s}, P_{a}=1 \mathrm{~Pa}\right) . \ldots . . . .$.

4.20 Simulated heating velocity profile comparisons for (top) orifice thruster geometry, and (bottom) MN-5 nozzle geometry $(\dot{m}=3.3 \mathrm{mg} / \mathrm{s}$, $\left.P_{a}=1 \mathrm{~Pa}\right) \ldots \ldots \ldots \ldots \ldots \ldots \ldots$

4.21 Simulated heating exit velocity plot comparison for the orifice and MN-5 nozzle geometries $\left(\dot{m}=3.3 \mathrm{mg} / \mathrm{s}, P_{a}=1 \mathrm{~Pa}\right) . \quad \ldots . . . \quad 74$

A.1 MKS Gas Calibration Curve for Series 275 Convectron Gauge [2]. . 84

A.2 InstruTech, Inc. CVG-101 Convection Gauge Calibration Table [3]. 85 


\section{NOMENCLATURE}

\begin{tabular}{|c|c|}
\hline$A$ & cross-sectional flow area \\
\hline$A_{e}$ & nozzle exit area \\
\hline$A_{t}$ & nozzle throat area \\
\hline$a$ & local acoustic velocity \\
\hline$C_{F}$ & coefficient of thrust \\
\hline$c_{p}$ & specific heat at constant pressure \\
\hline$c_{v}$ & specific heat at constant volume \\
\hline$e$ & elementary charge \\
\hline$F$ & thrust \\
\hline$g_{0}$ & acceleration due to gravity \\
\hline$I_{s p}$ & specific impulse \\
\hline$k$ & ratio of specific heat at constant volume and pressure \\
\hline$k_{c}$ & thermal conductivity \\
\hline$k_{B}$ & Boltzmann constant \\
\hline$L_{\text {cone }}$ & nozzle cone length \\
\hline$M$ & Mach number \\
\hline$M_{i}$ & ion mass \\
\hline$\dot{m}$ & mass flow rate \\
\hline$n_{0}$ & plasma density \\
\hline$P$ & power \\
\hline$P_{a}$ & ambient pressure \\
\hline$P_{c}$ & chamber pressure \\
\hline$P_{e}$ & orifice/nozzle exit pressure \\
\hline$P_{p l}$ & plenum chamber pressure \\
\hline
\end{tabular}




\begin{tabular}{|c|c|}
\hline$P_{\text {plume }}$ & plume power \\
\hline$P_{r f}$ & pre-matched RF input power \\
\hline$P_{s}$ & static pressure \\
\hline$P_{0}$ & total pressure \\
\hline$p d$ & pressure-distance \\
\hline$R$ & gas constant \\
\hline$R_{e}$ & nozzle exit radius \\
\hline$R_{t}$ & nozzle throat radius \\
\hline$T$ & temperature \\
\hline$T_{e}$ & electron temperature \\
\hline$T_{g}$ & neutral gas temperature \\
\hline$V$ & specific volume \\
\hline$V_{b r}$ & breakdown voltage \\
\hline$V_{P}$ & main plasma bulk potential \\
\hline$V_{P s}$ & plasma presheath potential \\
\hline$V_{W a l l}$ & wall potential \\
\hline$v_{B}$ & Bohm velocity \\
\hline$v$ & velocity \\
\hline$v_{e x}$ & exit velocity \\
\hline$\alpha$ & nozzle divergent half-angle \\
\hline$\gamma_{s e}$ & secondary electron emission coefficient \\
\hline$\epsilon$ & nozzle area ratio \\
\hline$\epsilon_{0}$ & permittivity of free space \\
\hline$\eta$ & efficiency \\
\hline$\lambda_{D}$ & Debye length \\
\hline$\rho$ & density \\
\hline
\end{tabular}




\section{Chapter 1}

\section{INTRODUCTION}

In order to provide a platform for low cost space exploration and university level education, Cube Satellites (CubeSats) were initially developed in 1999 by California Polytechnic State University-San Luis Obispo and Stanford University [4]. CubeSats are a class of nano-satellite that conform to standard dimensions of $10 \mathrm{x} 10 \mathrm{x} 10 \mathrm{~cm}$ ' $1 \mathrm{U}$ ' cubes that each weigh less than $1.33 \mathrm{~kg}$. These satellites are extendable to larger sizes between 2U-12U, and can house a large array of low Earth orbit and inter-planetary science mission payloads [5]. Since initial development, CubeSats have emerged as a collaborative industry that spans government, industry, and academia, demanding increases in functionality every year [4, 5]. Micro-propulsion systems have been developed to increase CubeSat payload capabilities and enable increasingly complex scientific experiments, by providing both main propulsion and attitude control systems $[5,6]$. In May of 2018, the first ever interplanetary CubeSats launched with the primary NASA mission, InSight. These CubeSats, known as MarCO-A and B, use a micro-propulsion system of eight cold gas thrusters that will provide attitude control and trajectory correction maneuvers for their data relay mission to Mars [7]. Historically, micro-propulsion options for CubeSats have largely been limited to the use of simple cold gas thrusters. There are currently many complex scaling issues associated with other propulsion options, both in terms of power requirements and system mass [5]. In order to provide an increase in performance over cold gas propulsion options, the Pocket Rocket thruster is currently under development as an electrothermal plasma micro-thruster. When compared to a cold gas thruster of similar size, Pocket Rocket can increase the exit thermal velocity of its gaseous propellant for increased thrust. Current thruster designs incorporate an orifice gas discharge tube for gaseous propellant expansion into the vacuum of space. Pocket Rocket's low power 
requirement of less than $10 \mathrm{~W}$ and its small mass make it a viable option for CubeSat missions [1]. Throughout this thesis, an experimental and computational study will investigate if the addition of a micro-nozzle, placed at the end of Pocket Rocket's gas discharge tube, will provide a further increase in thrust.

\subsection{Micro-Propulsion}

The primary mechanism that differentiates a propulsion system is its use of electrical power $[1,8]$. Split into two respective categories, electric propulsion (EP) systems are dependent on a continuous supply of power for operation, while non-electric, or chemical propulsion systems, may require only small amounts of segmented power to initiate and terminate operation [8]. Thrust $F$ and specific impulse $\mathrm{I}_{s p}$ are two key performance parameters used to design and characterize propulsion systems [9]. Thrust is due to the change in momentum of the propulsive device as it expels relatively small exhaust masses to very high velocities. The general thrust in the axial direction can be derived to be a combination of momentum thrust and pressure thrust as $[8]$

$$
F=\dot{m} v_{e x}+\left(P_{e}-P_{a}\right) A_{e}
$$

where momentum thrust consists of the propellant mass flow rate $\dot{m}$ and exhaust exit velocity $v_{e x}$. Pressure thrust is a function of the exhaust exit area $A_{e}$ and the difference between the exit pressure of the exhaust $P_{e}$ and the surrounding ambient fluid pressure $P_{a}[9]$. In the vacuum of space, $P_{a}$ can be approximated to equal zero $[8]$.

Specific impulse is a parameter that represents the change in momentum a propulsion device delivers per unit of consumed propellant. A higher value of specific impulse is desirable for better performance; it is an indication of how well the propulsive device uses its propellant $[8,9]$. Given the acceleration due to gravity $g_{0}$, with a constant 
mass flow rate and thrust, specific impulse can be defined in units of seconds as [8]

$$
I_{s p}=\frac{F}{\dot{m} g_{0}}
$$

In the case of large-scale EP systems, capable of maneuvering spacecraft upwards of thousands of kilograms, thrust is typically low $(<1 \mathrm{~N})$ with the advantageous capabilities of an extremely high specific impulse range (upwards of $3000 \mathrm{sec}$ ) [5, 8]. With the exception of electrothermal thrusters, EP devices use electrostatic and electromagnetic interactions to accelerate particles to high velocities to provide very low, but continuous thrust. This can often lead to heavy, high power sources $[1,8]$. Although the thrust to weight ratios of electric propulsion systems are not large enough to be useful within Earth's gravitational well, they are often ideal choices for deep space missions $[5,8]$. In contrast, large-scale chemical propulsion systems are capable of atmospheric use for lifting launch vehicles into orbit, as well as providing large spacecraft maneuverability [8]. Chemical propulsion systems rely on the thermal acceleration of gaseous propellant or combustion products through a nozzle, and have a much larger thrust range (starting at $\sim 1 \mathrm{~N}$ to upwards of $7000 \mathrm{kN}$ ) with much lower specific impulse capabilities $(<500 \mathrm{sec})$ [5]. As will be discussed further in section 1.1.2, electrothermal thrusters rely on gaseous thermal expansion through an orifice or nozzle for thrust, however, they are still classified as an electric propulsion system.

There are several key analysis and design considerations that must be taken into account when scaling down typical electric and chemical propulsion systems to the SmallSat and CubeSat range $[1,5,6]$. These considerations include the adaption to small mass and propellant storage capabilities, low power consumption requirements, and as it pertains to the work of this thesis, boundary layer losses within micronozzles $[10,6,11]$. Table 1.1 provides the range of performance values across various propulsion methods that are available for the SmallSat class, beginning with cold gas thrusters. 
Table 1.1: Range of performance values for micro-propulsion options with thrust $F$, specific impulse $I_{s p}$, and required power $P$.

\begin{tabular}{lllll}
\hline Thruster Type & $\mathrm{F}(\mathrm{mN})$ & $I_{s p}(\mathrm{~s})$ & $\mathrm{P}(\mathrm{W})$ & Refs. \\
\hline Cold Gas & $1-50$ & $40-75$ & - & {$[5,1,12]$} \\
MET & $0.2-1.4$ & $50-80$ & 6 & {$[13,14]$} \\
RFET & $0.01-0.1$ & $50-85$ & $10-60$ & {$[15]$} \\
Resistojet & $0.1-30$ & $48-150$ & $15-30$ & {$[5,1,12]$} \\
Arcjet & $0.014-0.031$ & $313-1200$ & $119-1000$ & {$[1,12]$} \\
Solid Rocket & $13-76$ & $187-270$ & - & {$[5,1,8,12]$} \\
Liquid Rocket & $1.5-1100$ & $214-256$ & - & {$[5,1,8,12]$} \\
RF Ion & $0.05-25$ & $300-3200$ & $28-145$ & {$[5,1,12]$} \\
Hall Effect & $1-50$ & $300-1750$ & $30-600$ & {$[5,1,12]$} \\
Electrospray & $0.005-1.5$ & $800-1800$ & $1.5-30$ & {$[1,16]$} \\
Pulsed Plasma & $0.001-0.144$ & $536-3000$ & $0.5-12.5$ & {$[5,1,12]$} \\
\hline
\end{tabular}

\subsubsection{Chemical Propulsion}

The most simple chemical propulsion type is the cold gas thruster, where no combustion takes place [8]. A cold gas system consists of a single propellant storage tank and a nozzle. Thrust is produced by the expansion of decompressed propellant through the nozzle into vacuum [8]. Although cold gas thrusters offer advantages in decreased system complexity, low power requirements, and low system mass, they are typically also the lowest performing method; specific impulse ranges start at approximately 30-40 seconds $[5,1]$. Common cold gas propellant choices for the SmallSat class of satellite include liquids that may be stored in very small volumes, such as hydrofluorocarbon refrigerants, before the compressed liquid is expanded and accelerated as a gas during operation [5]. Such is the propellant of choice for the MarCO cubes [7]. 
By adding thermal energy to a chemical propulsion system, thrust and specific impulse can be significantly increased [1]. In the case of liquid chemical thrusters, the selected propellant(s) provide a chemical combustion reaction that allows the thruster to transform the additional thermal energy into high kinetic exhaust energy [9]. Liquid propulsion thrusters may be of either the mono-propellant or bi-propellant variety. Mono-propellant thrusters use a catalyst bed to breakdown and ignite the selected propellant for combustion, whereas a bi-propellant thruster uses a fuel, oxidizer, and an initial ignition heat source to start combustion [8]. Bi-propellant thrusters may also use hypergolic propellants, in which spontaneous combustion occurs at initial propellant contact.

Solid chemical rocket motors operate on the same principle of liquid bi-propellant engines in that they require an oxidizer and a fuel for combustion; the difference being that the fuel and oxidizer is stored in a single, solid propellant grain [5]. The solid propellant grain acts as both the propellant storage and the combustion chamber, where combustion may be initiated by some pyrotechnic device that ignites the grain. Solid rocket motors typically have lower specific impulse performance when compared to bi-propellant thrusters, however they can greatly simplify the overall propulsion system design [8].

\subsubsection{Electric Propulsion}

Electrothermal, electrostatic, and electromagnetic thrusters are the three most basic types of electric propulsion systems [1]. Electrostatic and electromagnetic systems do not rely on the expansion of a propellant through a nozzle for thrust, and rely on vacuum conditions in order to generate a plasma for particle acceleration [12]. Although electrothermal thrusters may rely on gaseous thermal expansion for thrust, several electrothermal devices also require vacuum conditions for plasma generation [1]. 
Electrostatic thrusters provide thrust by accelerating ions to high velocities with the use of electrostatic fields [12]. In the case of ion and Hall-effect thrusters, xenon is typically used as the propellant of choice for its ability to provide heavy ions, and therefore higher momentum, for increased thrust. The accelerated ions must then be combined with electrons and neutralized after leaving the thruster chamber, in order to limit the buildup of electric charge on the spacecraft [12]. An ion engine works to electrostatically accelerate ions to high velocities through a system of charged grids, after the propellant is ionized by electrons emitted from a hollow cathode. The Hall thruster geometry traps electrons emitted from the cathode neutralizer in a magnetic field to ionize the propellant gas. Ions are then electrostatically accelerated to provide an increase in thrust and a slight decrease in specific impulse, as compared to ion engines [8]. Although ion and Hall thrusters provide ideal specific impulse performance, they typically require hundreds of Watts of power, making them poor options for low power CubeSat use. In addition to ion engines, various other electrostatic type thrusters have been of great recent interest $[5,16]$. These include electrospray thrusters, which accelerate ions that have been produced from electrified liquid surfaces $[16]$.

In an electromagnetic thruster, a hot gas's energized ions, neutrons, and electrons are accelerated to high velocities by both electric and magnetic fields [12]. Electromagnetic concepts include the pulsed plasma thruster, which accelerates a plasma by ablating a solid propellant, typically Teflon, with an electric discharge in a pulsing mode [5].

The electric propulsion type that is most similar in operating mechanics to chemical propulsion systems are electrothermal thrusters [8]. Types of electrothermal thrusters are arcjets, resistojets, and electrothermal plasma thrusters. Of the electrothermal plasma thruster varieties, power is typically coupled through either microwave $[13,14]$ or radio frequency [15] sources; denoted as METs and RFETs, re- 
spectively. All electrothermal propulsion concepts require a continuous supply of power, which is used to heat a propellant for increased performance over cold gas thrusters of similar size $[8,12]$. A resistojet directly heats a gaseous propellant by placing a heating element, such as a resistive coil, in the flow before the propellant is expanded through a nozzle [12]. Resistojets are typically limited by thermal fatigue and the thermal limit of the chosen thruster materials, as the thruster walls and heat exchanger elements are directly heated and cooled [5].

Arcjet thrusters, in comparison, typically allow for further increases in performance over resistojets by heating the propellant gas to higher temperatures than the thurster's material limits $[1,5]$. By using an anode and a cathode element, arcjets create a continuous electric discharge near the throat of the thruster's nozzle, thereby depositing thermal energy directly into the center of the propellant flow [12]. The main disadvantage of resistojet and arcjet systems is that they can require very large amounts of power, upwards of hundreds of Watts [1]. This typically makes arcjets and resistojets unsuitable for CubeSat propulsion systems, which may only have $<20$ W of total spacecraft power available [5].

Pocket Rocket, as an RF electrothermal plasma thruster, aims to also provide an increase in performance by depositing power directly into the propellant flow $[1,17]$. In this sense, it is much like an arcjet system, but with a much lower power requirement of $<10 \mathrm{~W}$. Unlike a resistojet system, the deposition of power directly into the propellant flow allows Pocket Rocket to obtain higher values of effective propellant heating, all while avoiding issues with material thermal fatigue [17]. As will be expanded on next, the main heating mechanism of Pocket Rocket relies on neutral particle interactions with the asymmetric generated plasma. 


\subsection{The Pocket Rocket Thruster}

The initial and on-going development of the Pocket Rocket thruster began in the Space Plasma, Power, and Propulsion laboratory at The Australian National University $[18,17]$. Pocket Rocket is an electrothermal thruster that uses radio-frequency (RF) to generate a weakly ionized and capacitively coupled plasma (CCP) [18, 19]. The mechanism for thrust largely relies on propellant heating via ion-neutral collisions in the plasma's discharge tube volume; the downstream heated propellant is then thermodynamically expanded into the vacuum of space to provide a propulsive force [1]. In order to provide an understanding of this particular thrust mechanism, a review of the key plasma physics topics will first be discussed.

\subsubsection{Plasma Theory}

Matter can exist in four distinct forms; solid, liquid, gas, and plasma [20]. A plasma is an ionized gas that contains freely moving electrons and ions, and is described as being electrically quasi-neutral on a macroscopic scale. Given the free motion of electric charges in a plasma, particles can create varying local electromagnetic fields and respond collectively to applied external fields [21, 20]. Interestingly, plasma is the most common type of verified matter in the universe, and can exist over a broad range of frequencies, temperatures, and densities [21]. The relevant plasma theory for this work will consist of the characteristics of weakly ionized, low pressure plasma, generated by a RF signal.

Although a plasma is defined as macroscopically having an equal number of positive and negative charges, small regions of local charge imbalance can exist [20]. Once a local charge imbalance has formed, oppositely charged particles will be attracted to that region, effectively shielding the bulk of the plasma from the charge disturbance. 
This shielding distance is characterized as a fundamental plasma property called the Debye length $\lambda_{D}$ as

$$
\lambda_{D}=\sqrt{\frac{\epsilon_{0} T_{e}}{e n_{0}}}
$$

where $\epsilon_{0}$ is the permittivity of free space, $T_{e}$ is the electron temperature, $e$ is the elementary charge, and $n_{0}$ is the plasma density [20].

A gas can be ionized to achieve a plasma state by applying an electric field, which will accelerate free electrons in the gas in the direction parallel to the applied field. The electrons which obtain a high enough ionizing potential can then ionize neutral particles in the gas to create ion-electron pairs in an exponential process [21]. In the case of Pocket Rocket's operation, an applied alternating current (AC) will accelerate electrons back and fourth as polarity alternates. This increases the possibility of ionizing passes for each electron, as compared to an applied direct current (DC) field [1]. Upon initiating DC gas ionization, each electron is lost to the anode or container walls for a total of one potential ionizing pass [20]. In order to initiate a plasma breakdown with an applied RF field, a minimum breakdown voltage $V_{b r}$ must be achieved. This voltage is dependent on the pressure-distance $p d$ from Paschen's Law as

$$
V_{b r}=\frac{B p d}{\ln (A p d)-\ln \left(\ln \left(1+\frac{1}{\gamma_{s e}}\right)\right)}
$$

where $A$ and $B$ are experimentally determined constants for a particular gas (related to the constituents' excitation and ionization energies) and $\gamma_{s e}$ is the secondary electron emission coefficient [20]. An example Paschen curve for argon gas can be seen in Figure 1.1, where the minimum breakdown voltage occurs at $114.8 \mathrm{~V}$ and 4.7 Torr-mm. 


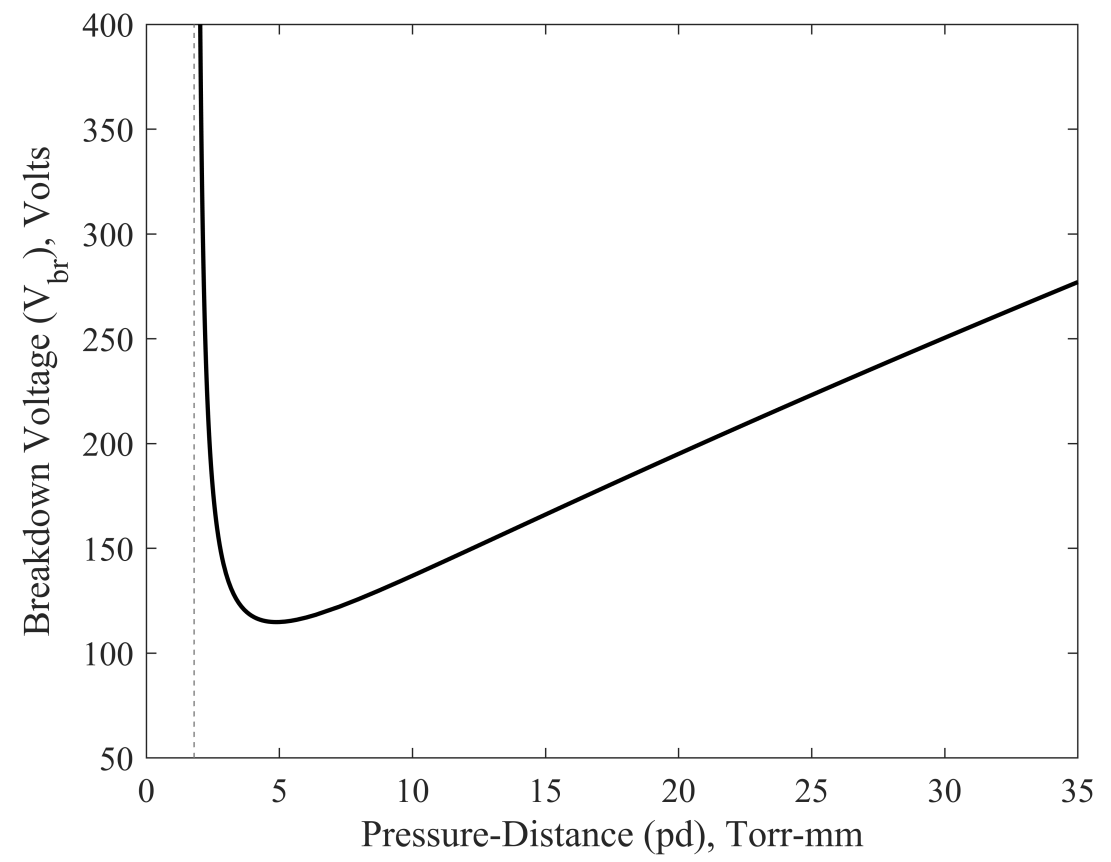

Figure 1.1: Paschen curve for argon gas.

\subsubsection{Sheath Breakdown}

The thermal velocity of an electron is at least 100 times that of an ion, which correlates to much greater mobility [22]. Therefore, upon initial plasma breakdown, a large flux of these fast electrons will be lost to the anode and plasma container walls before any slower ions are able to travel the same distance. This in turn creates a positive space charge within the plasma bulk that is separated from the container walls by a very sharp drop in potential [20]. This potential drop is known as a sheath, and acts as a mechanism for both retaining electrons in the plasma bulk and accelerating ions towards the container walls [20].

Figure 1.2 depicts generic sheath regions with respect to potential vs. distance away from the plasma container wall; $V_{p}, V_{P s}$, and $V_{W \text { all }}$ are the potentials at the main plasma bulk, presheath, and wall, respectively. Ion density within the sheath will be greater than electron density, with quasi-neutrality returning in the presheath 


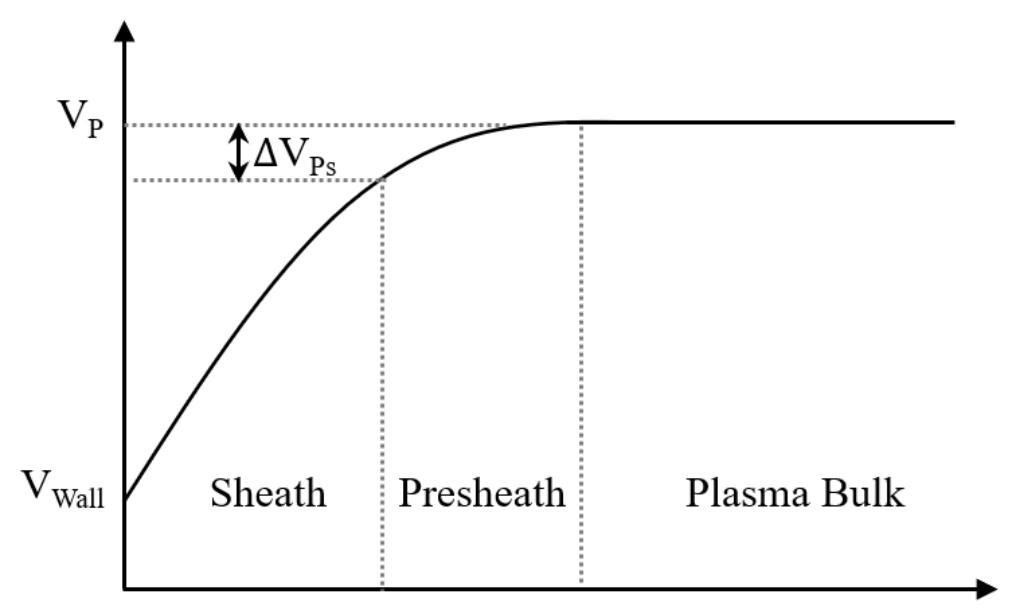

Figure 1.2: Generic sheath regions in a plasma with main plasma bulk potential $V_{p}$, presheath potential $V_{P s}$, and wall potential $V_{W a l l}$.

and main plasma bulk regions [21]. Since the flux of electrons which make it to the container walls have a significantly greater thermal energy, the ions must enter the sheath region at a specific velocity. This allows the sheath region to maintain equal fluxes of electrons and ions. The ion velocity entering the sheath region is called the Bohm velocity $v_{B}$ and is a function of electron temperature $T_{e}$ and ion mass $M_{i}$ as

$$
v_{B}=\sqrt{\frac{e T_{e}}{M_{i}}}
$$

Sheath regions are typically several Debye lengths thick, with a presheath region that acts as the initial potential gradient before the sheath edge [20]. The potential drop across this presheath region can be given as $\Delta V_{P s}=\frac{T_{e}}{2}[1]$.

\subsubsection{Secondary Electrons}

Once the ions that have been accelerated through the plasma sheath reach the container walls, they may impact in such a way that releases a secondary electron back into the discharge through the Auger process. This can be described as the release of an electron on the surface material when excess energy from impact neutralization 
is higher than the surface's work-function [21]. This secondary electron may result in an increase in ionization [1]. A coefficient $\gamma_{s e}$ can be used to indicate how many electrons may be emitted per ion impact, and plays an important secondary role in the neutral gas heating mechanism of Pocket Rocket [1].

\subsubsection{Capacitively Coupled Plasma}

In a typical CCP system, operation is biased by a single RF power supply at 13.56 MHz. The plasma is formed between two parallel electrodes, but is separated from each electrode by space-charge sheaths that vary in thickness corresponding to the RF used [21]. Given the sheath at the RF electrode and sheath at the grounded electrode, the voltage distribution can be thought of as two capacitors in series, and can be modeled as such [1]. The oscillating sheath potentials accelerate electrons in the plasma for power deposition, resulting in ionization sub-modes $\alpha$ and $\gamma$. In the $\alpha$ mode, plasma bulk electrons are responsible for ionizing the gas via direct collisions, while the ionization in the $\gamma$ mode comes from secondary electrons produced from wall collisions [21]. Due to the excited molecules in the gas, light emission can be directly observed in Pocket Rocket's CCP plasma.

\subsubsection{Thruster Operating Principles}

Performance in Pocket Rocket is improved over a cold gas thruster of similar size because of the propellant heating that occurs before gaseous expansion into vacuum [1]. Figure 1.3 depicts a cross-sectional view of the thruster.

A propellant gas (typically argon) is fed into a $2 \mathrm{~mm}$ gas inlet and into a $40 \mathrm{~mm}$ inner diameter, $70 \mathrm{~mm}$ outer diameter, and $12 \mathrm{~mm}$ long plenum chamber, where the flow stagnates. A low vacuum pressure gradient then expands the plenum chamber gas through a central gas discharge tube, where the propellant is heated by an RF 


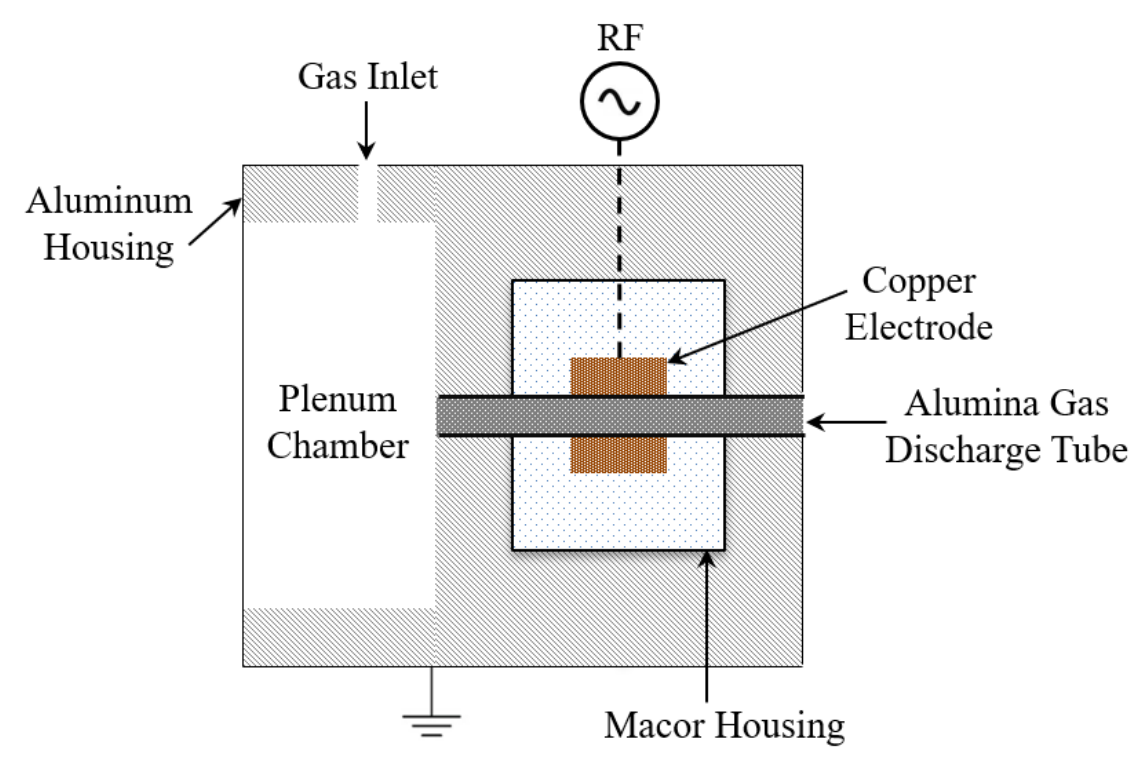

Figure 1.3: Pocket Rocket cross-section schematic.

generated, asymmetric and weakly ionized CCP plasma [1]. The gas discharge tube is a 18-20 mm long, $4.2 \mathrm{~mm}$ inner diameter, $6.3 \mathrm{~mm}$ outer diameter alumina tube that acts as a dielectric [1]. The alumina material provides a high secondary electron coefficient for more efficient propellant ionization. Surrounding the discharge tube is a powered copper electrode and copper feed-through pin which provides a 13.56 $\mathrm{MHz} \mathrm{RF}$ signal, nominally at $240 \mathrm{~V}$ and $10 \mathrm{~W}$ for propellant ionization. This 5 $\mathrm{mm}$ wide, $12 \mathrm{~mm}$ diameter copper electrode and discharge tube is surrounded by a $15 \mathrm{~mm}$ diameter glass Macor housing, which separates the powered copper electrode from the grounded electrodes, as well as provides thermal insulation for the discharge tube. Once powered, the $60 \mathrm{~mm}$ diameter aluminum thruster housing acts as the grounded electrodes, wrapping around the ends of the gas discharge tube to complete the circuit for plasma generation [1]. 


\subsubsection{Neutral Gas Heating in Pocket Rocket}

The gas heating mechanisms in Pocket Rocket have been characterized by measuring the neutral gas temperature of the thruster discharge with rovibrational spectroscopy [1]. In this work, volume averaged steady state gas temperatures were measured over the operating conditions and an analytic model was developed to verify experimental results $[1,23]$. In addition, computational fluid dynamics (CFD) simulations were performed using the CFD-ACE + software package. Experimental results showed that neutral gas heating in Pocket Rocket can reach up to $1060 \mathrm{~K}$ for argon and $430 \mathrm{~K}$ for nitrogen at the standard operating conditions of $10 \mathrm{~W}$ power and 1.5 Torr plenum pressure. Most notably, spatiotemporal profiles of the gas temperature indicate that the dominant heating mechanisms of Pocket Rocket are ion-neutral collisions in the discharge tube, along with ion bombardment to the thruster walls [1]. Simulation results demonstrate that the discharge is driven by secondary $\gamma$ electrons emitted from the plasma cavity by ion bombardment. The emitted electrons are accelerated through a developed plasma sheath, and create a peak in ion density in the center of the gas discharge tube. The preliminary thruster performance results, as calculated using the ANU Pocket Rocket's measured neutral gas temperatures $T_{g}$, can be seen in Table $1.2[1]$.

Table 1.2: Preliminary performance estimates for Pocket Rocket [1], with neutral gas temperature $\mathbf{T}_{g}$, mass flow rate $\dot{m}$, exit exhaust velocity $v_{e x}$, specific impulse $I_{s p}$, thrust $F$, plume power $P_{\text {plume }}$, and efficiency $\eta$.

\begin{tabular}{|c|c|c|c|c|c|c|c|}
\hline & $\mathrm{T}_{g}(\mathrm{~K})$ & $\dot{m}\left(\mathrm{mg} \mathrm{s}^{-1}\right)$ & $v_{e x}\left(\mathrm{~ms}^{-1}\right)$ & $I_{s p}$ & $\mathrm{~F}(\mathrm{mN})$ & $P_{\text {plume }}(\mathrm{W})$ & $\eta(\%)$ \\
\hline Argon & 1060 & 3.3 & 750 & 76 & 2.4 & 0.9 & 9.2 \\
\hline Nitrogen $\left(N_{2}\right)$ & 385 & 5.6 & 540 & 55 & 3.0 & 0.8 & 8.2 \\
\hline
\end{tabular}

Thrust was calculated as momentum thrust using Equation 1.1, and propellant exit velocity was assumed to be ejected at the constant thermal exhaust velocity as 


$$
v_{e x}=\sqrt{\frac{8 k_{B} T_{g}}{\pi M}}
$$

where $M$ is the molecular mass of the propellant and $k_{B}$ is the Boltzmann constant.

Efficiency $\eta$ has been calculated as $\frac{P_{r f}}{P_{\text {plume }}}$, where $P_{\text {plume }}$ is the plume power and $P_{r f}$ is the pre-matched RF input power of $10 \mathrm{~W}$. Preliminary performance estimates have indicated that Pocket Rocket has similar performance with argon propellant as METs and RFETs, as indicated in Table $1.1[13,14,15]$. Slightly lower efficiency values are a result of high losses observed in the matching network and RF generation sources [1]. With these losses addressed in an optimized configuration, Pocket Rocket remains as a highly viable micro-electrothermal plasma thruster option for CubeSat missions.

As will be discussed in section 3.1, the Cal Poly thruster [24] used in this work will use a different power system and a slightly different, more modular design than the ANU built thruster. This will allow for increased modularity for micro-nozzle experimentation. However, due to these differences, the estimates in Table 1.2 will not be directly comparable to the experimental thruster used in this work.

\subsection{Basic Nozzle Theory}

A propulsive nozzle is a device used to control the expansion of gas from high pressure to low pressure and accelerate the gaseous propellant, or combustion products, to high exit velocities. By using Newton's Third Law, relative motion is created when matter is ejected out of a nozzle, thereby producing an opposite force that is applied to both the combustion chamber and nozzle walls [9]. In terms of spacecraft use, this thrust is usable for activities such as attitude control, station keeping, orbit raising and lowering, as well as interplanetary exploration $[5,7]$.

Pocket Rocket's current configuration does not include an optimized nozzle located 
after the gas discharge tube [1], although concurrent research is investigating the performance increase associated with placing a nozzle inside of the gas discharge tube, near the RF electrode $[25,26]$. This thesis will look at how a micro-nozzle may affect thruster performance, when placed at the end of the gas discharge tube. Beginning with the present section, basic nozzle theory and geometry will be presented. A more detailed introduction on selecting the pertinent nozzle design parameters and isentropic relations will be given in Chapter 2. Following section 1.3, a review will be given on the previous research that has been preformed on micro-nozzle optimization. The full thesis scope will then be presented in section 1.5.

\subsubsection{Nozzle Geometry}

There are three basic nozzle shapes that can improve a propulsion system's performance given the flow parameters of the expected gas or combustion products; diverging, converging, and converging-diverging [9]. Converging nozzles are typically used by aircraft jet engines to increase exit velocity from subsonic flow to a maximum exit Mach number equal to 1, while diverging nozzles are used on hypersonic vehicles when the flow is already supersonic [8]. For chemical rocket propulsion, a converging-

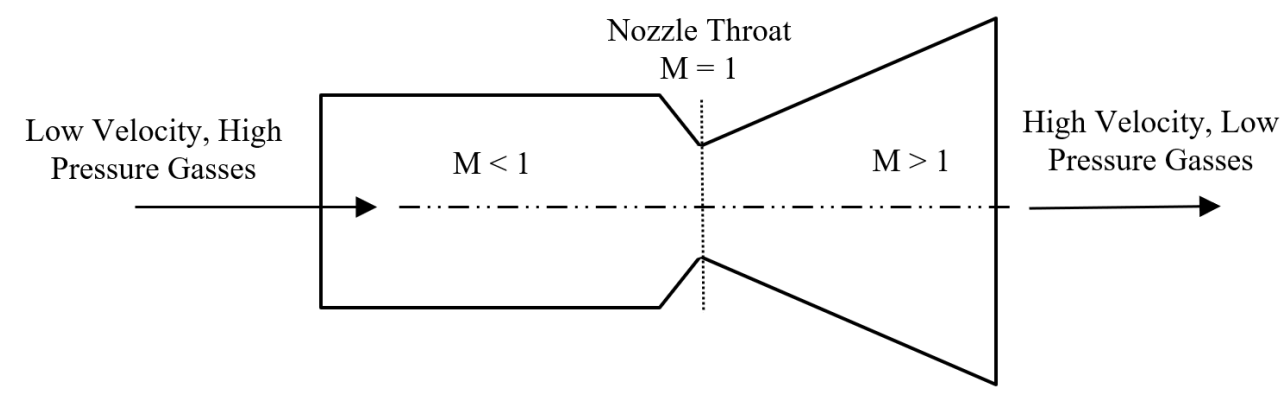

Figure 1.4: Converging-Diverging nozzle concept.

diverging (CD), or De Laval nozzle, is used to take subsonic flow and convert it into supersonic flow. This process involves converting the available thermal energy from the exhaust gas into kinetic energy [8]. A simple schematic in Figure 1.4 shows the 
CD nozzle basic working principles. Initially at subsonic speeds, the conservation of mass requires the fluid velocity to increase as it flows through the constricting section of the nozzle throat. The Venturi effect then causes the static pressure and density to decrease downstream of the nozzle throat restriction, thereby accelerating the flow [8]. In all supersonic CD nozzles, the Mach number at the throat must be equal to 1, where flow velocity is exactly equal to the local speed of sound. This condition, called choking, is the condition at which the mass flow rate through the nozzle will no longer increase with any further decrease in downstream pressure $[8,9]$.

The nozzle design for Pocket Rocket takes advantage of a simple, conical nozzle geometry that will produce the least amount of manufacturing variability. While bellshaped nozzles almost always result in higher performance for large-scale propulsion systems, previous research has indicated that for flows with Reynolds number $R e<$ $1 \times 10^{5}$, performance test results have been ambiguous between conical and bell-shaped micro-nozzles [27]. This is in large part due to micro-nozzle boundary layer growth. Figure 1.5 shows several key design characteristics for a conical nozzle shape, namely the diverging half angle $\alpha$ and the nozzle cone length $L_{\text {cone }}$. Another important geometrical parameter for a nozzle is the definition of the ratio of exit area $A_{e}$ to throat area $A_{t}$, defined as $\epsilon=\frac{A_{e}}{A_{t}}[8]$. The area ratio can be found from the respective dimensions for exit radius $R_{e}$ and throat radius $R_{t}$, as seen in Figure 1.5.

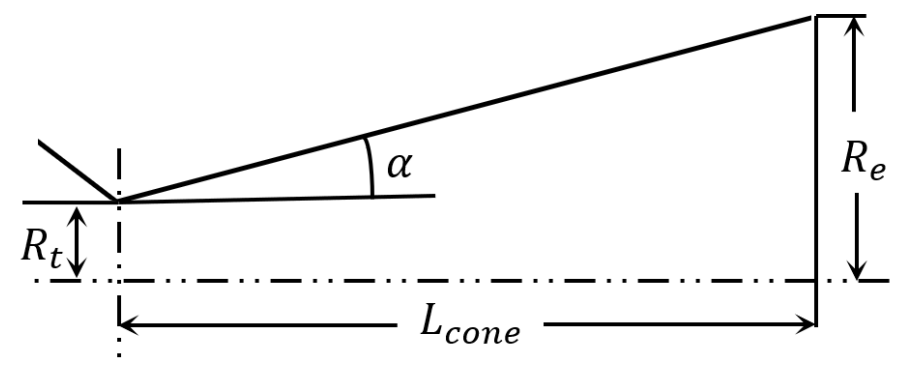

Figure 1.5: Conical nozzle geometry. 


\subsection{Previous Studies on Micro-Nozzle Optimization}

Nozzles for propulsion systems producing thrust on the order of $\mathrm{mN}-\mu \mathrm{N}$ have typically been classified as micro-nozzles, with varying throat diameters on the scale of millimeters [11]. Flows within micro-nozzles have been continually characterized since the 1960's. In 1968, TRW systems tested heated hydrogen and nitrogen propellants, up to $1088 \mathrm{~K}$, within several micro-nozzles [28]. This research, using nozzle throat diameters of $2 \mathrm{~mm}$, found that the optimum half angle for Reynolds number flows in the 600-3000 range was approximately $\alpha=20^{\circ}$, with an area ratio of $\epsilon=100$. Both the TRW study and a NASA study from 1987 [29] found that nozzle flows with a Reynolds number less than 1000 had large performance hindering boundary layer losses in the nozzle throat and diverging sections. This NASA study, using nozzles with throat diameters between 0.6-0.7 mm, also concluded that the viscous losses associated with low Reynolds number flows may vary largely with the gaseous species used. Additionally, it was observed that the divergent section geometry of the nozzles tested had little effect on the specific impulse efficiency [29]. Here, specific impulse efficiency is defined as the ratio of measured specific impulse to the expected specific impulse assuming isentropic expansion.

In research done by LaTorre in 2011, two micro-nozzle geometries were presented; $\alpha=20^{\circ}$ with a sharp angle between the converging and diverging sections, and $\alpha=15^{\circ}$ with a smooth throat section transition [11]. LaTorre found that the micronozzle with a larger half angle and sharper throat had the highest performance. By minimizing the nozzle cone length and surface area throughout the throat transition region, boundary layer losses can be minimized. In general, this study found that efficiency losses due to viscous boundary layers within micro-nozzles lead to a decrease in efficiency of up to $10 \%$. Wall roughness can add an additional $10 \%$ in losses. In order to obtain the optimal performance from a micro-nozzle system, it was concluded 
that the divergent half angle should be larger than what is typical on optimized large scale nozzles $\left(>\alpha=15^{\circ}\right)[11]$.

Most recently, a study done by the Naval Research Laboratory tested micronozzles within a range of low Reynolds numbers and nitrogen gas temperatures; between $\mathrm{Re}=65-807$ and 295-523K, respectively [10]. In this study, the authors once again found that micro-nozzles with larger half angles and smaller area ratios were more efficient, leading to the highest observed specific impulse values. By minimizing the area ratio, boundary layer interactions are limited, but at the cost of the reduction in the maximum specific impulse that is achievable. In general, nozzles with larger area ratios have a higher specific impulse due to higher isentropic expansion, but have increased viscous losses [10]. The larger nozzle half angle also allows for a shorter overall cone length for the same area ratio desired, which provides less space for boundary layer growth. As gas temperature increases, theoretical performance increases as there is more internal energy available that can be converted into kinetic energy. However, as this study concludes, increasing the temperature in a real system also raises viscosity and therefore increases loss effects in the nozzle [10]. Although specific impulse is increased with increasing gas temperatures, the overall result is one that is lower than an ideal inviscid system. Another notable result of this study is that the authors found the transition from $423 \mathrm{~K}$ to $523 \mathrm{~K}$ in nitrogen propellant produced similar increases in specific impulse with negligible decreases in nozzle efficiency. From this result, it possible that increasing gas temperatures would further provide increases in specific impulse without a decrease in efficiency. In particular, this may be possible if the gas transitions from the continuum flow regime to a free molecular flow regime, where gas-surface interactions dominate the flow [10].

Concurrent micro-nozzle studies for Pocket Rocket specifically are also on-going at ANU $[25,26]$. In research done by Ho, CFD-ACE + simulations have been completed with sculpted nozzle designs within Pocket Rocket's discharge tube, in which the 
sculpted nozzle design was based on the highest performing geometry from LaTorre's research $[25,11]$. This study found that the CFD results indicate an increase in plasma confinement, and therefore propellant heating, that provides an increase in thrust by $30 \%$ during plasma operation at 10W. Ho concluded that additional plasma sheaths are able to form at the micro-nozzle's throat, thereby providing an increase in plasma confinement [25].

In order to compliment concurrent studies at ANU, this thesis will seek to answer whether further increases in nozzle divergent half angles and area ratios will provide an increase in thruster performance.

\subsection{Thesis Scope}

This thesis is an experimental and computational study aimed at discovering if the addition of a micro-nozzle, placed at the end of the gas discharge tube in the Pocket Rocket thruster, will increase performance. Previous research has indicated that the addition of a micro-nozzle in a propulsion system (with throat diameter on the order of $<1 \mathrm{~mm}$ ) may actually decrease performance, as large viscous boundary layers can dominate the flow through the nozzle throat. However, by designing a nozzle in such a way that boundary layer losses are minimized, performance can still be greatly increased [11, 28, 10, 29]. Most of these previous studies have only characterized micro-nozzle flows for 1-2 different nozzle geometries, using very specific ranges of Reynolds number flows. As of yet, none of the previous studies have tested argon propellant flow conditions that is directly comparable to the exact operating conditions Pocket Rocket will use. This leaves the question of micro-nozzle performance optimization in Pocket Rocket unanswered.

In this work, an experimental nozzle is fabricated and placed at the end of the gas discharge tube of a previously built [24] Cal Poly Pocket Rocket thruster. Performance 
is characterized for both the original orifice discharge tube thruster configuration and the nozzle configuration with the use of a pitot-static tube. By measuring static and total pressure at various locations within the exhaust plume, performance of the thruster's Mach number can be characterized with the $P_{s} / P_{0}$ ratio, and exit velocity may be estimated. As previous studies have indicated that larger micronozzle divergence half-angles help to minimize performance hindering boundary layers in the flow, the experimental nozzle is designed to provide an $\alpha=30^{\circ}$ and an $\epsilon=10$. This conical nozzle geometry is in the middle of the range of various nozzle geometries that are simulated with CFD.

Experimental results for both the orifice and nozzle thruster configuration are used to validate steady state CFD simulations performed in CFD-ACE + . With the validation of the CFD simulations, a matrix of 9 different nozzles with varying geometries are simulated in order to characterize which geometry may be best suited to increase thruster performance. The 9 micro-nozzle geometries simulated are designed to produce a wide range of performance results; with $\alpha=15^{\circ}-60^{\circ}$, and $\epsilon=$ 5-100. With the the experimental and computational results, future work can quickly determine the exact optimized nozzle geometry required for any one specific Pocket Rocket mission. 
Chapter 2

MICRO-NOZZLE DESIGN METHODOLOGY

\subsection{Design Parameters and Isentropic Flow Relationships}

\subsubsection{Mach Number}

The local flow velocity $v$ and local acoustic velocity $a$ is used to determine a dimensionless nozzle design parameter called the Mach number. Since the local acoustic velocity, or velocity of sound, in a perfect gas is independent of pressure as $a=\sqrt{k R T}$, the Mach number can be described as

$$
M=\frac{v}{a}=\frac{v}{\sqrt{k R T}}
$$

where $\mathrm{k}$ is the ratio of the gas's specific heat at constant pressure and volume, $\mathrm{R}$ is the gas constant, and $\mathrm{T}$ is the absolute fluid static temperature [8]. For a Mach number equal to 1, Equation 2.1 shows that flow is precisely moving at the velocity of sound, indicating a condition called sonic flow. For a Mach number less than or greater than 1, the flow is described as subsonic and supersonic, respectively [8].

The Pocket Rocket thruster relies on thrust generation via the expansion of neutral gas in a discharge tube into the low pressure vacuum of space. The propellant initially starts in a near stagnant state in the plenum chamber with pressure $P_{p l}$, before it is expanded through the discharge tube and into an ambient vacuum pressure at $P_{e}$

[1]. In order to achieve choked flow, this pressure ratio $\frac{P_{p l}}{P_{e}}$ must reach a critical value determined by the choked flow criterion as [8]

$$
\frac{P_{p l}}{P_{e}}=\left(\frac{k+1}{2}\right)^{\frac{k}{k-1}}
$$

The choked flow criterion can be reached in all ideal gasses for $\frac{P_{p l}}{P_{e}} \geq 2.1[1]$. Pocket 
Rocket will operate in the vacuum of space with ambient pressures in Low Earth Orbit (LEO) of $10^{-8}$ to $10^{-10}$ Torr. Although this will lead to pressure gradients greater than $10^{8}$, the mass flow rate in the gas discharge tube will not change significantly for $\frac{P_{p l}}{P_{e}} \geq 2.1$. This in turn allows for experiments to be carried out at much lower vacuum pressures in the $10^{-1}$ to $10^{-3}$ Torr range [1].

\subsubsection{Isentropic Flow}

The isentropic nozzle flow equations can be applied to the Pocket Rocket thruster to analyze performance, as very little plasma is actually ejected from the thruster. Any plasma bulk that is ejected is so weakly ionized $(<1 \%)$ that ideal thermodynamic relations are still accurate [1]. These relations allow for the evaluation and prediction of performance for any propulsion system that uses the thermodynamic expansion of a gas to design key elements, such as nozzle size and shape [8, 9].

An isentropic process is an idealized process where the entropy of the system remains unchanged. By not accounting for shocks or friction, the flow entropy change may be zero, and the conservation of energy may be applied for an adiabatic and reversible flow process inside of a nozzle [8]. Coupling this theory with the conservation of mass and enthalpy within a flow system, the isentropic flow equations can be developed.

Within a nozzle, the conservation of mass requires that any two mass flow rates $\dot{m}$ at varying locations $x$ and $y$, will be equal for a single inlet and outlet flow process $\dot{m}_{x}=\dot{m}_{y}=\dot{m}[8]$. This gives rise to the continuity equation for mass flow rate

$$
\dot{m}=A v / V=\rho V A
$$

where $A$ is the cross-sectional area, $v$ is the velocity, and $V$ is the specific volume. The definition of enthalpy consists of the internal thermal energy of a system plus 
the flow work. In the case of nozzle flow, the flow work is the work performed by an ideal gas, and can be expressed as the absolute temperature $T$ multiplied by the the specific heat of the gas at constant pressure $c_{p}[9]$. The specific heat ratio $k=\frac{c_{p}}{c_{v}}$ at constant pressure and constant volume for an ideal gas remains constant over a very wide temperature range, giving rise to the isentropic flow relation between any two points in a nozzle as [8]

$$
\frac{T_{x}}{T_{y}}=\left(\frac{p_{x}}{p_{y}}\right)^{(k-1) / k}
$$

Stagnation conditions with subscript " 0 " for $P$ and $T$ denote the values for each respective variable as if the the flow was stopped isentropically, and can be computed from Mach number as

$$
\begin{gathered}
T_{0}=T\left[1+\frac{1}{2}(k-1) M^{2}\right] \\
P_{0}=P\left[1+\frac{1}{2}(k-1) M^{2}\right]^{k /(k-1)}
\end{gathered}
$$

For a nozzle with isentropic flow, the expansion area ratio between any two points in a CD nozzle can be related through Mach number as

$$
\frac{A_{y}}{A_{x}}=\frac{M_{x}}{M_{y}} \sqrt{\left[\frac{1+[(k-1) / 2] M_{y}^{2}}{1+[(k-1) / 2] M_{x}^{2}}\right]^{(k+1) /(k-1)}}
$$

When given the exit Mach number from a nozzle $M_{2}$, the ideal nozzle area ratio $\epsilon$ can be determined by setting Mach number at the throat $M_{t}=1$ [8].

\subsubsection{Coefficient of Thrust}

The thrust coefficient is a key nozzle design parameter that can be used to determine an optimal area ratio [9]. It is based on the ratio of chamber pressure $P_{c}$ across the pressure at the nozzle exit $P_{e}$, ratio of specific heat $k$, and nozzle area ratio $\epsilon$. Equation 2.8 gives the thrust coefficient $C_{F}$ as a dimensionless parameter.

$$
C_{F}=\sqrt{\frac{2 k^{2}}{k-1}\left(\frac{2}{k+1}\right)^{(k+1) /(k-1)}\left[1-\left(\frac{P_{e}}{P_{c}}\right)^{(k-1) / k}\right]}+\frac{P_{e}-P_{a}}{P_{c}} \epsilon
$$


The thrust coefficient, and therefore thrust of any system, will be at a maximum when $P_{e}=P_{a}$ for any fixed pressure ratio $\frac{P_{c}}{P_{a}}$. The thrust coefficient can also further simply Equation 1.1 for thrust to be [8]

$$
F=C_{F} A_{t} P_{c}
$$

Since $C_{F}$ is directly proportional to the nozzle throat area $A_{t}$, this parameter can provide information on the increase in thrust due to the addition of gas expanding through a supersonic nozzle, as compared to the thrust force that would occur through just the nozzle throat area [8]. As $\frac{P_{c}}{P_{a}}$ becomes extremely large for gas expanding into vacuum, the thrust coefficient will reach an asymptotic maximum at some nozzle area ratio. Figure 2.1 shows the thrust coefficient curve for argon gas, which will be the propellant used for Pocket Rocket in this work.

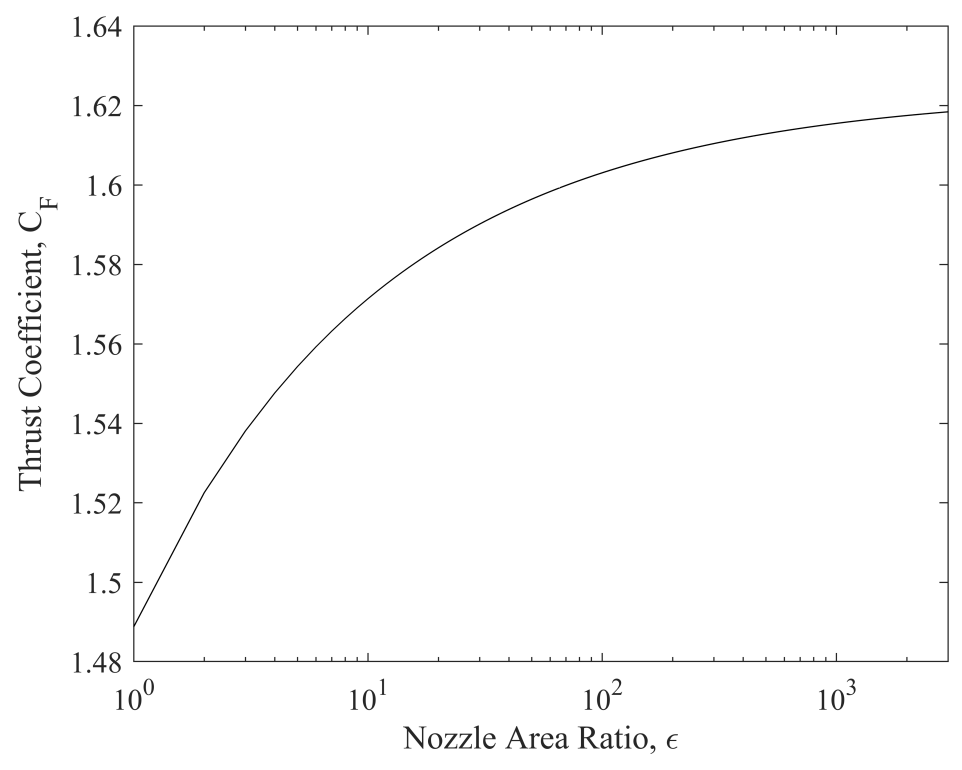

Figure 2.1: Nozzle area ratio vs. coefficient of thrust for argon gas.

The thrust coefficient curve is often used to select an appropriate area ratio in nozzle design [9]. Although a larger $\epsilon$ corresponds to an increase in thrust, overall system performance may be hindered due to an increase in nozzle mass. Performance 
can also be hindered as a result of flow separation for mismatched nozzles with large area ratios, under conditions where the exit pressure does not equal the ambient pressure. Area ratios along the knee of the thrust coefficient curve are often chosen as the optimal design criterion; where the performance increase from any further increase in $\epsilon$ is negligible for the selected system performance goals. The knee of this curve provides a compromise between increases in system mass and larger thrust coefficients [9].

There are two cases for nozzle performance conditions when $P_{e} \neq P_{a}$; underexpanded and over-expanded [8]. An over-expanded nozzle may cause flow separation within the nozzle's divergent section, as the exhaust exits at a lower pressure than the ambient atmosphere. In this case, the area exit is too large to produce an optimal amount of thrust. The opposite is true for an under-expanded nozzle, where the exhaust $P_{e}$ is greater than $P_{a}$. In this case, there will not be flow separation, but expansion waves will form downstream of the nozzle exit as the gas continues to expand into the lower pressure atmosphere [8].

\subsection{Geometry Selection}

Theoretically, the most optimal nozzle is one that isentropically converts all available thermal energy into kinetic energy via expansion of a gaseous propellant [8]. This can be achieved by lowering the exhaust exit pressure to directly match the ambient pressure. Such a nozzle operating in near perfect vacuum conditions would require an infinitely long cone length, and therefore area ratio $\epsilon$, given some divergence half angle. An infinitely long nozzle is not only impossible, but larger cone lengths can start to reduce the performance of a system by increasing the vehicle mass and design

complexity [9, 8]. Typically, smaller nozzle divergence half angles allow for increased specific impulse with an increase in thrust in the axial direction [8]. However, these 
nozzles also require a longer nozzle cone length. This can be seen in the trigonometric relation for a conical nozzle cone length as

$$
L_{\text {cone }}=\frac{R_{e}-R_{t}}{\tan (\alpha)}
$$

where $R_{e}$ and $R_{t}$ is the radius at the nozzle exit and throat, respectively, and $\alpha$ is the diverging half angle.

A large divergence half angle will typically provide short and lightweight nozzle designs, but performance can significantly decrease. The optimum diverging half angle for large-scale nozzle propulsion systems is typically $\alpha=15^{\circ}$ [8]. However, as previously discussed, micro-nozzles with throat diameters on the order of $1 \mathrm{~mm}$ or less have been found to result in better performance with larger half angles, thereby minimizing boundary layer flow losses. [11, 28, 10].

In order to characterize what effect the diverging half angle and area ratio would have on nozzle performance for Pocket Rocket, a matrix consisting of a wide range of varying geometries was developed. These geometries will be used as the CFD simulation test cases. The various geometries for a conical shaped nozzle can be seen in Table 2.1. The half angles of $\alpha=15^{\circ}, 30^{\circ}$ and $60^{\circ}$ were chosen in order to bound performance results for a large range of increasing half angles, which previous research has suggested plays a critical role in minimizing diverging viscous boundary layer losses [11].

The area ratios of 5,10 , and 100 were chosen in order to fully characterize the knee of the thrust coefficient curve from Figure 2.1. Characterizing the knee of this curve allows for a nozzle design selection that compromises on increased design mass and complexity, due to an increase in nozzle area ratio, that would otherwise provide a negligible increase in thrust [9].

The selection of the conical nozzle geometry was chosen as the most simple design that would produce the least amount of variability in manufacturing, and therefore 
Table 2.1: Simulation cases for varying micro-nozzle conical geometries with diverging half angle $\alpha$, area ratio $\epsilon$, throat radius $R_{t}$, and nozzle cone length $L_{\text {cone }}$.

\begin{tabular}{lllll}
\hline Geometry Name & $\alpha\left(^{\circ}\right)$ & $\epsilon$ & $R_{t}(\mathrm{~mm})$ & $L_{\text {cone }}(\mathrm{mm})$ \\
\hline MN-1 & 15 & 5 & 1.38 & 6.34 \\
MN-2 & 15 & 10 & 0.97 & 7.85 \\
MN-3 & 15 & 100 & 0.31 & 10.33 \\
MN-4 & 30 & 5 & 1.38 & 2.94 \\
MN-5 & 30 & 10 & 0.97 & 3.64 \\
MN-6 & 30 & 100 & 0.31 & 4.79 \\
MN-7 & 60 & 5 & 1.38 & 0.98 \\
MN-8 & 60 & 10 & 0.97 & 1.21 \\
MN-9 & 60 & 100 & 0.31 & 1.59 \\
\hline
\end{tabular}

test repeatability, compared to more complicated bell shaped contours.

In order validate the simulation cases from Table 2.1, the MN-5 nozzle geometry of $\alpha=30^{\circ}, \epsilon=10$ was selected to be manufactured and tested. The MN-5 test geometry represents the middle of the range of selected geometry variations, and manufacturing details can be found in section 3.1.1. 
Chapter 3

\section{THRUSTER EXHAUST FLOW CHARACTERIZATION}

\subsection{Thruster Hardware}

The thruster components used for experimentation were previously manufactured in order to conduct research on the electrode sizing effects on plasma density [24]. The thruster's effective modularity made for a good candidate that provided an easily interchangeable end-plate for nozzle testing. However, this thruster differs from the original ANU thruster schematic shown in Figure 1.3 in several key ways. These changes can be seen in Figure 3.1.

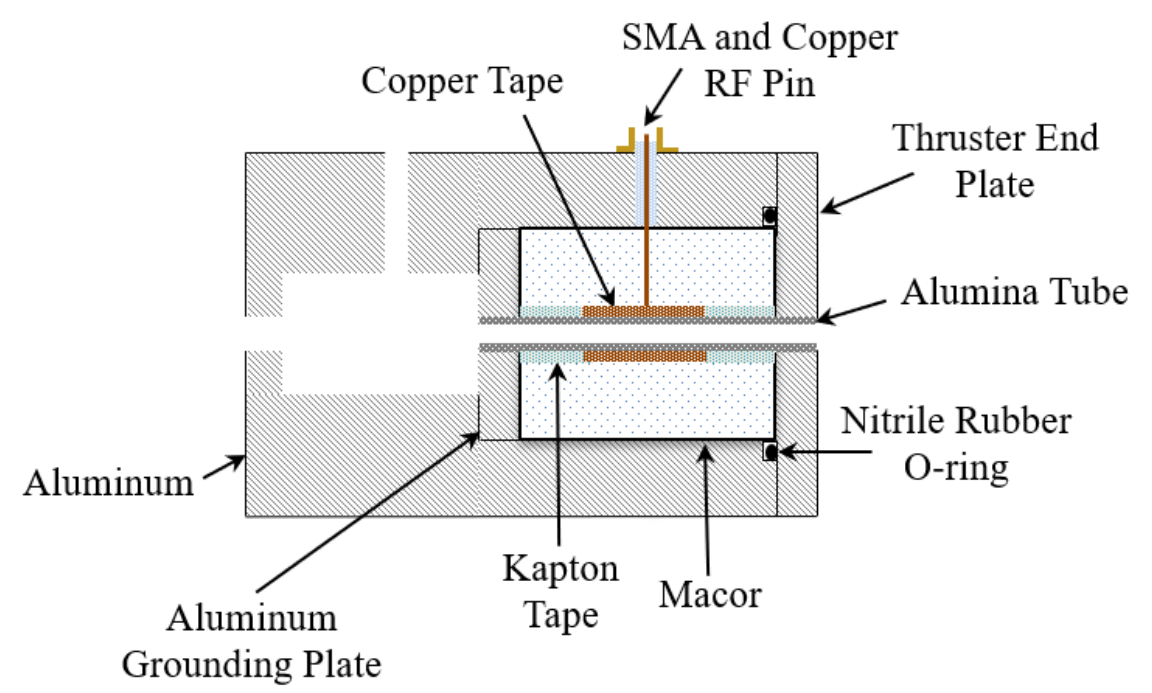

\section{Figure 3.1: Schematic cross-section for modular thruster.}

Instead of the $5 \mathrm{~mm}$ wide annular copper electrode, the discharge tube uses 4.93 $\pm 0.1 \mathrm{~mm}$ of copper tape wrapped around the center of the tube. A copper wire is soldered onto an SMA RF connection to provide the RF feed-through pin. The copper tape is surrounded by $4.5 \mathrm{~mm}$ of Kapton tap and $3 \mathrm{~mm}$ of grounded aluminum housing on both sides of the discharge tube. The Kapton tape was used in an effort to 
prevent any parasitic discharge between the RF electrode and the grounded electrodes [24]. The thruster housing itself is also slightly smaller than the full scale version, and uses a $12.8 \mathrm{~mm}$ diameter plenum chamber that is $15 \mathrm{~mm}$ deep. Silicone tubing is used to connect the gas inlet and pressure port to hose barbs on the thruster plenum exterior [24]. All fabricated thruster interior components can be seen in Figure 3.2.

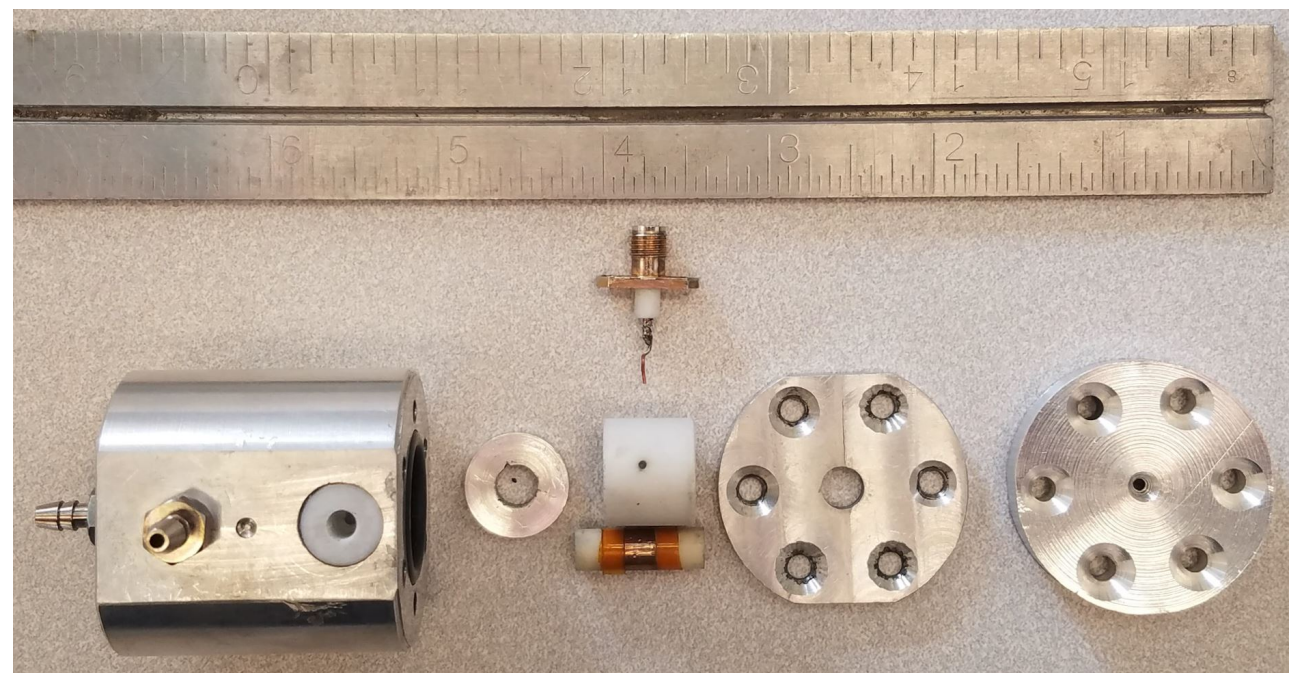

Figure 3.2: Modular Pocket Rocket components (from left to right); aluminum housing with plenum chamber barbed connections, aluminum grounding ring, SMA connector/Macor housing/alumina gas discharge tube, original thruster orifice end-plate, $\mathrm{MN}-5$ nozzle end-plate.

Separating the aluminum thruster housing and end plate is an o-ring, which is compressed by $6 \mathrm{M} 4$ flat-head screws. This end-plate also acts as the grounded electrode that wraps around $3 \mathrm{~mm}$ of the protruding alumina gas discharge tube.

\subsubsection{Nozzle Fabrication}

The MN-5 nozzle was machined out of 3 inch cylindrical aluminum stock on a lathe to an overall final diameter of of $1.505 \pm 0.005$ inches. The aluminum piece was then faced to a width of $0.248 \pm 0.005$ inches. A standard $1 / 4$ inch drill bit with a point angle of $135^{\circ}$ was then used in the lathe center point holder in order to drill into one side of the aluminum face, for a total distance of $0.139 \pm 0.0005$ inches. The 
aluminum piece was then flipped around, and a 6 flute $60^{\circ}$ countersink was installed in the lathe center holder. The countersink was drilled into the center of the aluminum piece for a total of $0.111 \pm 0.0005$ inches, until a final nozzle exit diameter of 0.184 \pm 0.005 inches, and nozzle throat diameter of $0.061 \pm 0.005$ inches was obtained. Finally, 6 holes were drilled and countersinked into the aluminum face to house the 6 M4 end-plate compression screws.

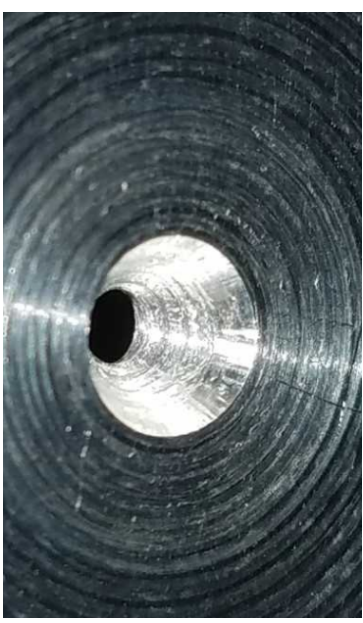

(a)

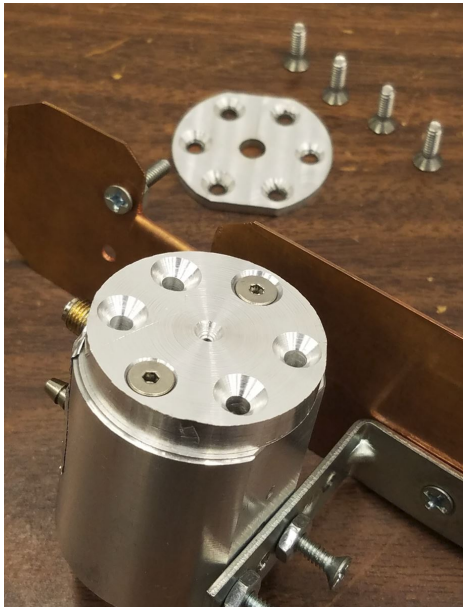

(b)

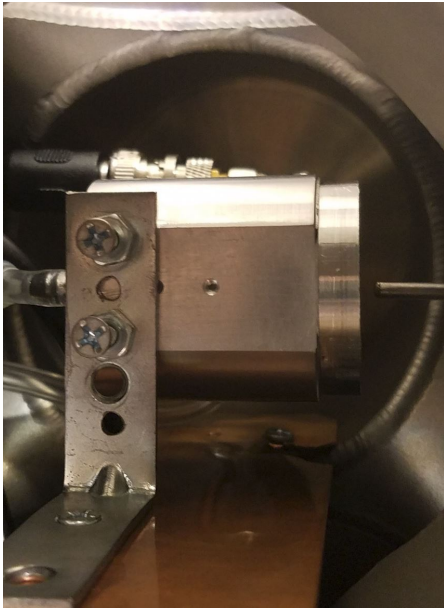

(c)

Figure 3.3: (a) MN-5 machined nozzle close-up, (b) Nozzle to thruster installation with original orifice end-plate in the background, (c) Thruster to vacuum chamber installation.

The final machined nozzle, nozzle to thruster installation, and thruster to vacuum chamber installation can be seen in Figure 3.3. The standard 1/4 inch drill bit with a point angle of $135^{\circ}$ was used to create the converging angle of the nozzle, for a final converging half angle of $67.5^{\circ}$. This converging half angle was chosen for ease of manufacturing, as previous research has indicated that the converging half angle size has negligible affects on overall micro-nozzle performance [29]. The $1 / 4$ inch bit also left a straight bore that wraps around the end of the discharge tube to act as the final thruster grounded electrode. The 6 flute $60^{\circ}$ countersink was used to create the desired nozzle diverging half-angle of $30^{\circ}$. 
Overall, the final machined MN-5 nozzle ended up with an area ratio of $9.1 \pm$ 0.086, compared to the target area ratio of 10 .

\subsection{Test Setup}

Testing took place in Cal Poly's small 6-sided CF style vacuum chamber, which includes a six inch top view port window, RF electrical, and gas feed-through ports. Using a rotary-vane roughing pump, the chamber is pumped down to vacuum ranges between $10^{-4}$ to $10^{-3}$ Torr before thruster operation. In the test setup diagram shown in Figure 3.4, all pressure measurements were taken with convectron gauges: The plenum pressure, vacuum chamber pressure, and pitot total pressure ports used MKS Granville-Phillips 275 gauges, while the pitot static port used an InstruTech Worker Bee CVG-101 convectron gauge. With the exception of the pitot-static tube, all connections used 1/4 inch Swagelok tubing and feedthroughs.

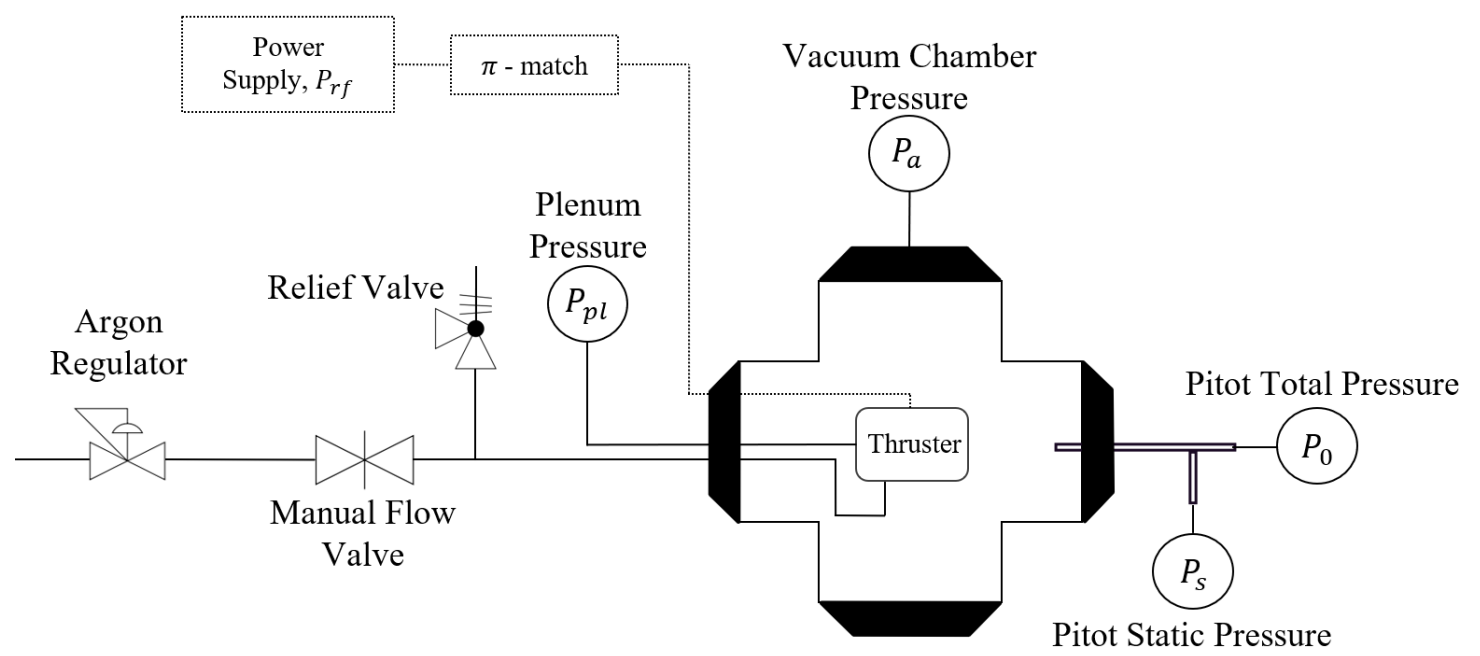

Figure 3.4: Experiment piping and instrumentation diagram.

A manual flow needle valve was used in combination with a pressure regulator, as the argon regulator was not designed to accurately control pressures on the order of 
the required 1.5 Torr thruster plenum pressure. A full picture of the test setup can be seen in Figure 3.5.

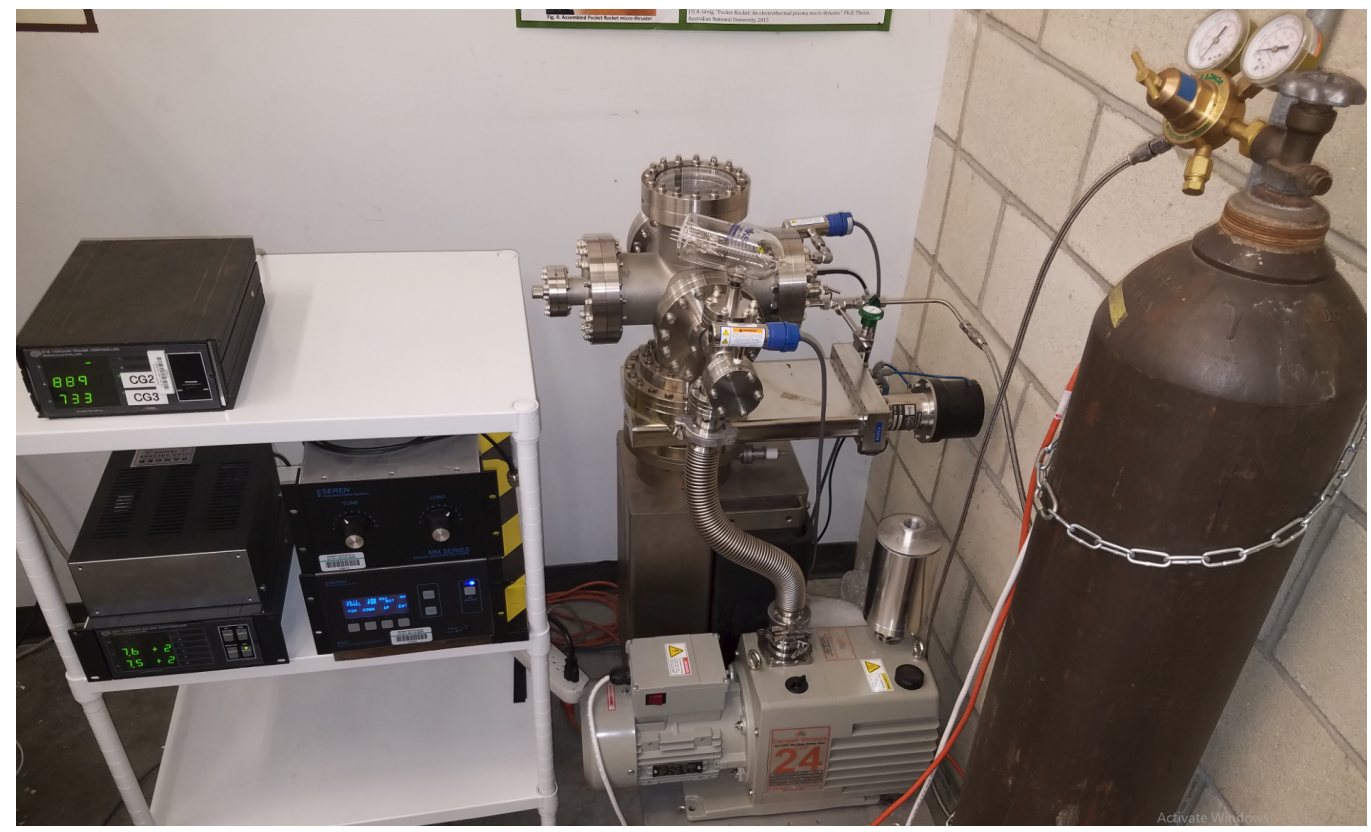

Figure 3.5: Experiment electrical equipment, vacuum chamber, rotary pump, and propellant feed system.

Ten watts of power was supplied to the thruster by a Seren R301 power supply, set at a frequency of $13.56 \mathrm{MHz}$. A Seren MM300 matching network was used to match the impedance of the system to the $50 \Omega$ of the power supply. During thruster operation, the matching network was adjusted to display $0 \mathrm{~W}$ of reflected power. Power from the matching network was sent through an N-Type cable to a male to female, N-Type to BNC vacuum feed-through. Inside the chamber, a BNC cable was used to route power to the thruster, using an SMA adapter at the end to connect to the thruster.

\subsubsection{Pitot-Static Tube Instrumentation}

Performance measurements to characterize an electrothermal thruster can be obtained through several different methods [30]. The implementation of a load-cell or thrust balance was considered, however, both methods of collecting only direct thrust data 
would not allow for full characterization of the exhaust plume for exact CFD flow field comparisons.

In order to directly characterize Pocket Rocket's exhaust flow, a Dwyer model 166$6,1 / 8$ inch pitot-static tube was implemented to obtain both total and static pressure at various locations within the thruster plume. By using the ratio for static and total pressure, Equation 2.6 can be used to directly calculate Mach number under isentropic assumptions, assuming a constant ratio of specific heat $k$ [8]. The pressure ratio can also be used to directly compare between the experiment results and CFD simulation flow field. This eliminates the need to make any additional isentropic assumptions for performance estimations, such as constant ratio of specific heat, temperature, or density.

Typically, pitot tubes measure differential pressure using a capacitance manometer, or physical fluid U-shaped or inclined manometer. The differential pressure can then be used to calculate the fluid velocity, given a constant density, by using Bernoulli's equation [31]. However, convectron gauges were used as the connections to the total and static pressure ports in this work. Capacitance manometers that can measure a large enough differential pressure range to allow for the measurement of vacuum pressures on the order of $10^{-3}$ to several Torr, are extremely costly. In particular, these manometers must retain the high resolution required to measure the subtle changes in the downstream pressures of Pocket Rocket. Additionally, most digital manometer devices only give a reading output for differential pressure, instead of separating total and static pressure components. Having only access to the differential pressure measurement forces the observer to make an incompressible flow assumption in order to calculate velocity [31]. By implementing convectron gauges with the pitot tube, a low cost and effective exhaust characterization method can be realized, which allows for separate recordings of static and total pressures within the flow. This pitot tube integration with the vacuum chamber can be seen in Figure 3.6. 


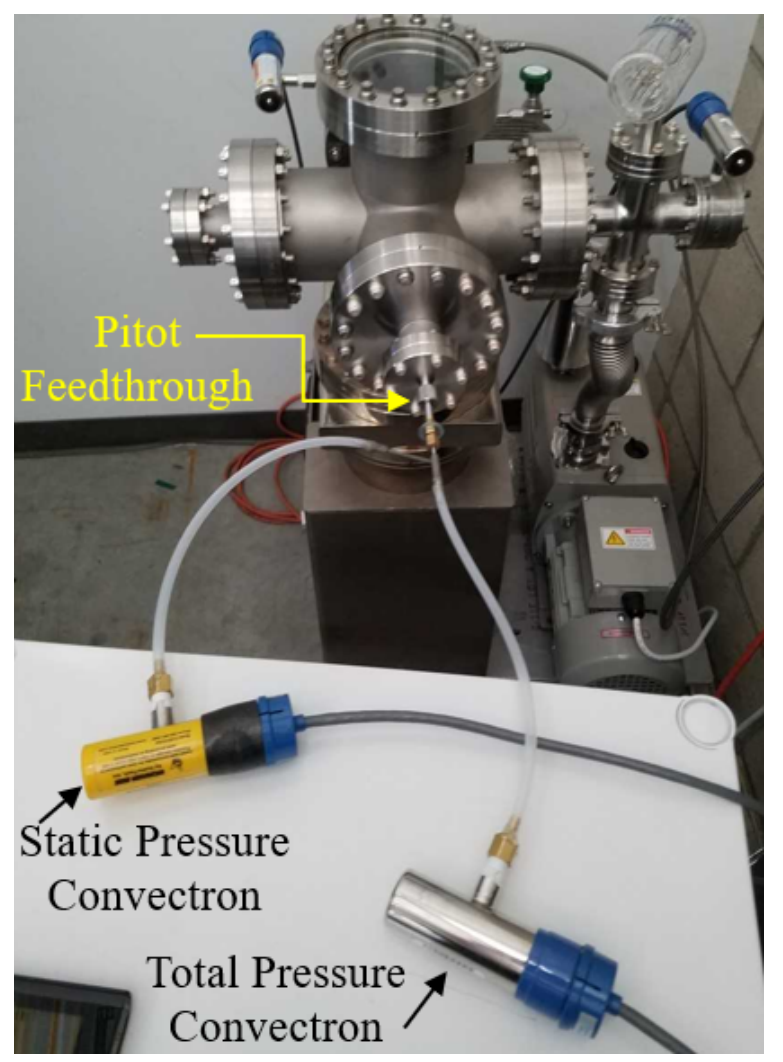

Figure 3.6: Pitot-static tube with convection gauges.

The $1 / 8$ inch pitot tube was sealed through a $1 / 4$ inch hollow aluminum tube with the use of an epoxy. The pitot and hollow tube system was then inserted into the chamber via an adjustable 1/4 inch Quick-Connect coupling with and o-ring seal. In an effort to prevent epoxy out-gassing effects, the end of the tube that would be exposed to vacuum was coated in a purple PVC pipe primer with a much lower outgassing rate [32]. PTFE standard tubes were then connected to the the total and static pitot tube ports, where a small amount of epoxy was again used to create a vacuum seal. The ends of the PTFE tubes were connected to the convectron gauges via a barbed hose to $1 / 8$ inch NPT adapter. Although the use of exposed epoxy and PTFE tubing to vacuum would give rise to out-gassing concerns in high vacuum experiments, the operational pressures used in this work of $>10^{-1}$ Torr make it of little concern [24]. An adjustable test stand structure, which can be seen in Figure 
3.3(c), was used in order to align the thruster's exit exhaust flow jet concentrically with the pitot tube position.

\subsubsection{Testing Procedure}

Two testing campaigns were completed; one with the thruster in its original orifice end-plate configuration, and one with the MN-5 nozzle end-plate configuration. For each campaign a total of 10 test runs were performed, both in the cold gas state and during plasma operation. A test run consisted of taking total and static pressure measurements at various locations in the thruster exhaust plume, from 0.05 inches to 3 inches, where a reference point of 0 inches marked the exit plane of the thruster. More measurements were taken between 0.05-0.5 inches than between 0.5-3 inches in order to characterize the rapidly changing flow just after the thruster exit. Readings were not recorded at the exact exit plane of the thruster, as the $1 / 8$ inch pitot tube itself was observed to block the flow through the discharge tube, inhibiting normal gas expansion.

Before each test run, the vacuum chamber was evacuated to $\sim 10^{-4}$ Torr. The plenum chamber pressure was then set to an indicated value of either 1.5 Torr or 2.5 Torr for the orifice configuration and the MN-5 nozzle configuration, respectively. An arbitrary higher plenum pressure was run with the nozzle configuration since the nozzle throat will choke a smaller mass flow rate, and therefore result in smaller pressure ratio differences within the exhaust plume, than the orifice configuration. By increasing the flow through the nozzle, changes in the downstream pitot-static pressures are more readily observable on a similar scale as that of the orifice configuration. With gas now flowing through the thruster discharge tube and into the vacuum chamber, ambient pressure typically sat at an indicated value of 0.57 Torr for the orifice configuration, and 0.27 Torr for the nozzle configuration. 
Although the indicated values of 1.5 and 2.5 Torr were used in order to match previous Pocket Rocket experimentation as closely as possible, a correction factor was required for both the InstruTech and MKS convectron gauges, which were calibrated for air and $N_{2}$ use only $[2,3]$. Since each convectron gauge relies on the specific thermal conductivity and heat capacity of the gas for which it is calibrated for, using argon gas with these gauges requires the use of a correction factor for each measurement. The InstruTech manufacturer provides a table of calibration correction factors [3], while the MKS manufacturer provides an empirical graph of correction factors for argon [2]. Both the correction table and graph used can be found in the Appendix of this work. Therefore, the actual plenum pressures that were used in experimentation were $2.9 \pm 0.5$ Torr and $6.1 \pm 0.5$ Torr for both the orifice and nozzle campaigns, respectively. The ambient actual vacuum pressures were then $0.9 \pm 0.05$ Torr and $0.45 \pm 0.05$ Torr for the orifice and nozzle test runs, respectively.

For both cold gas and plasma operation test runs, the pitot tube started away from the thruster exit at 3 inches. The pitot tube was then increasingly brought closer to the exit at the same traverse intervals for each test. Dial calipers with English units were used to measure pitot tube axial adjustment in order to ensure accuracy down to \pm 0.005 inches. At each measurement location, pressure readings were recorded only after a steady state change in pressure was indicated. The time to a steady state pressure typically took anywhere from 1-2 minutes. For plasma operation test runs, the thruster was allowed to run for 5 minutes before measurements were taken, in order to ensure that the thruster had reached a steady thermal state with respect to wall heating [25]. 


\subsection{Experiment Results}

In order to build confidence that 10 test runs at each thruster plume measurement location would be adequate to give a normal distribution of data, probability plots of the $\frac{P_{s}}{P_{0}}$ pressure ratio were created. Figure 3.7 shows a sample probability plot for cold gas flow with the MN-5 nozzle at 3 inches $(76.2 \mathrm{~mm})$ and 0.05 inches $(1.27 \mathrm{~mm})$ away from the thruster exit. The cold flow sample at 3 inches from the thruster exit

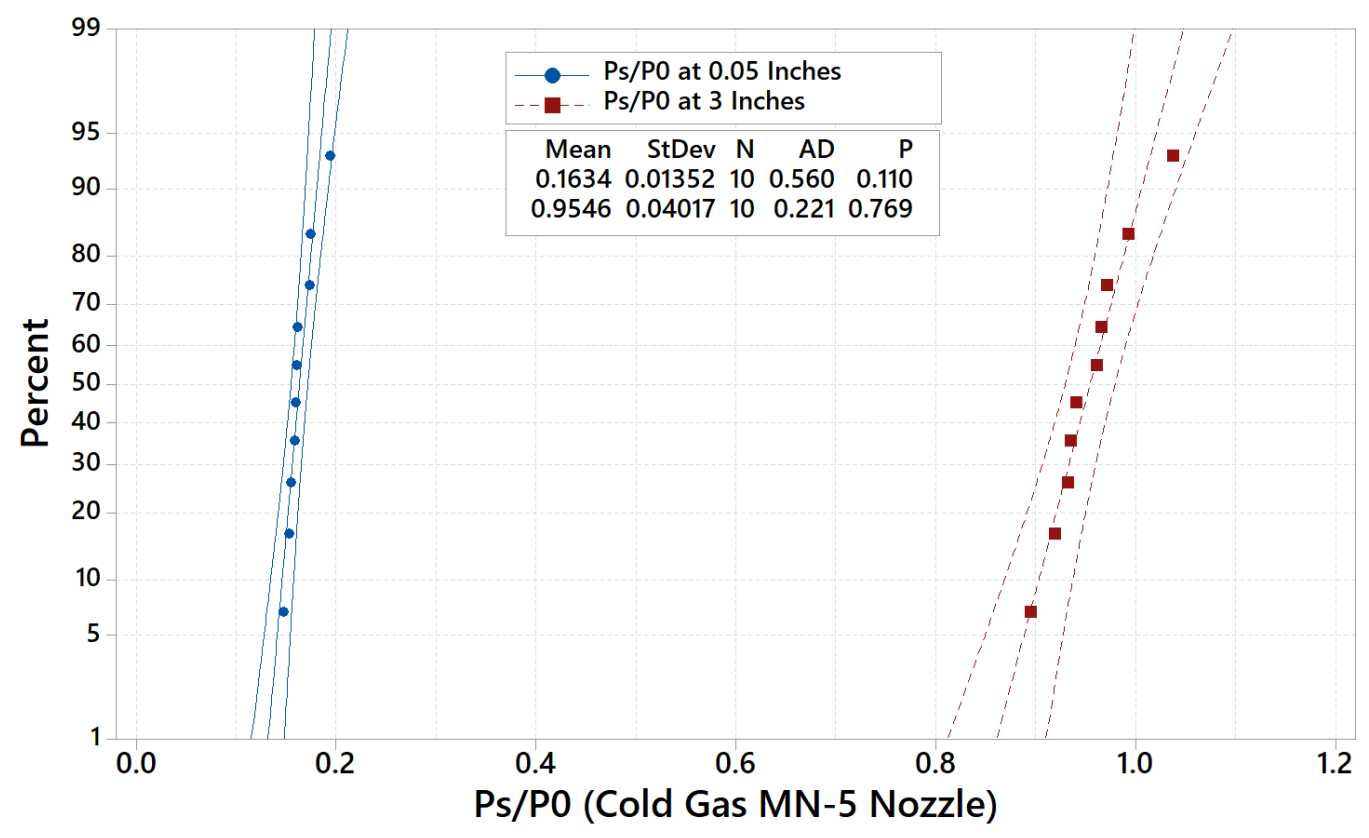

Figure 3.7: Normal distribution probability plot with 95\% confidence intervals for cold gas MN-5 nozzle $\frac{P_{s}}{P_{0}}$ measurements at 0.05 inches from the thruster exit (blue circles) and 3 inches from the thruster exit (red squares).

is significant for two reasons; the pitot tube is no longer visible through the vacuum chamber view-port, and deflections from the plasma plume are not present to indicate any misalignment's associated with the pitot tube and the exhaust flow. Therefore, this pitot location and test condition will give rise to the highest errors in the collected data. However, the probability distribution plot in Figure 3.7 indicates a reasonable standard deviation of 0.04 for the measured pressure ratio. 
The sample closest to the thruster exit at 0.05 inches shows a much smaller standard deviation of 0.014 . This is expected, as the pitot tube is directly observable in relation to the thruster exit at 0.05 inches. Figure 3.7 also indicates P-Values of 0.769 and 0.110 for the data collected at 3 inches and 0.05 inches from the exit, respectively. Both P-Values are not small enough to reject the null hypothesis that the data follows a normal distribution, at a standard significance level of $5 \%$.

\subsubsection{Orifice Thruster Configuration Testing}

Measurements from the orifice thruster configuration can be seen in Figure 3.8, during both cold gas and plasma operation. Vertical error bars on each plot represent $3 \sigma$ of standard deviation of the measured values, with the combined error in uncertainty due to the application of the convectron gauge correction factor. Error bars can be seen to generally increase for both test cases. This is most likely a result of the increase in the chances of misalignment with the thruster plume as the pitot tube moves further away from the thruster exit.

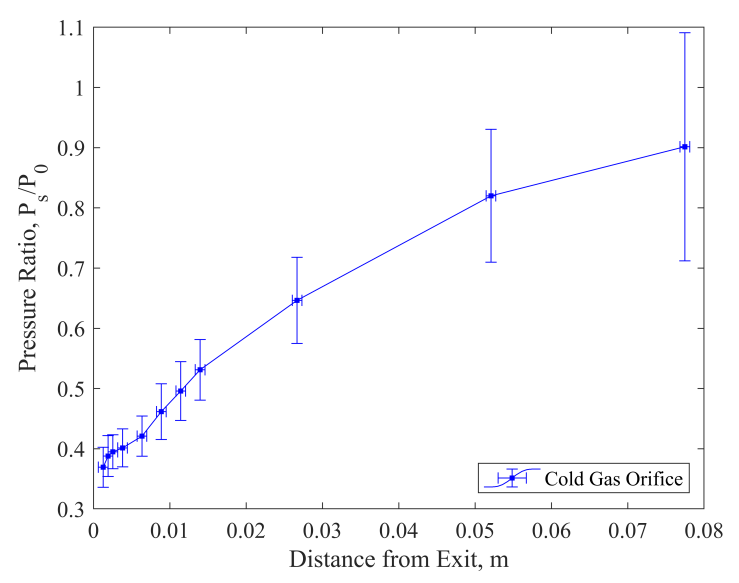

(a)

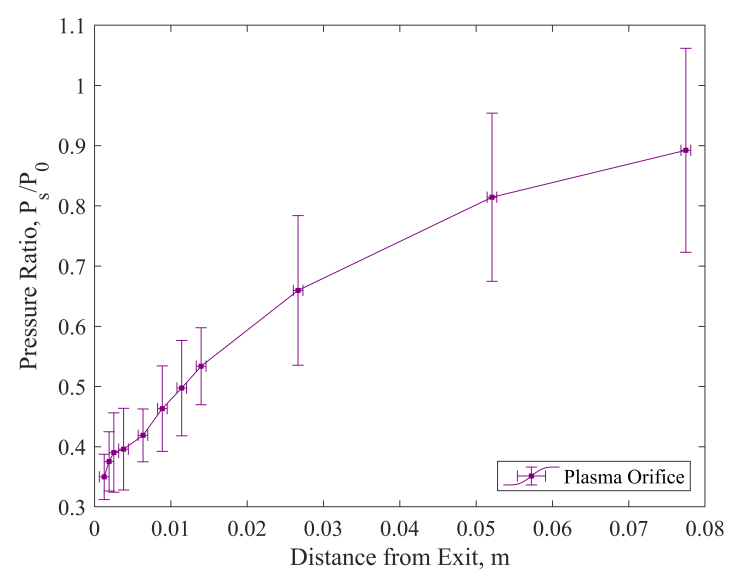

(b)

Figure 3.8: Orifice experimental results for (a) cold gas testing, and (b) plasma operation testing $\left(P_{p l}=2.9 \pm 0.5\right.$ Torr, $P_{a}=0.9 \pm 0.05$ Torr $)$. Vertical error bars represent $3 \sigma$, horizontal error bars represent $0.64 \mathrm{~mm}$ of parallax error. 
Figure 3.9 shows the thruster plume interactions with the pitot tube at various locations, which was a good indication of exact pitot alignment with the flow. These pictures were taken under different lighting conditions, with different auto exposure and white balance settings, in order to highlight the pitot tube in the flow. Therefore, the plume brightness cannot be directly compared between tests in Figure 3.9.

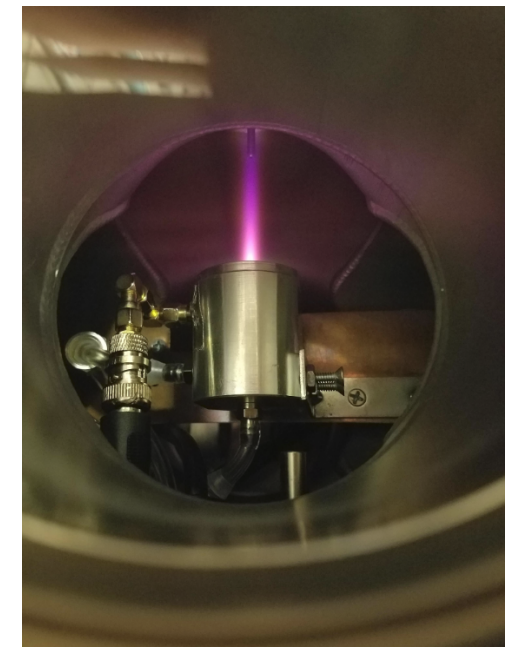

(a)

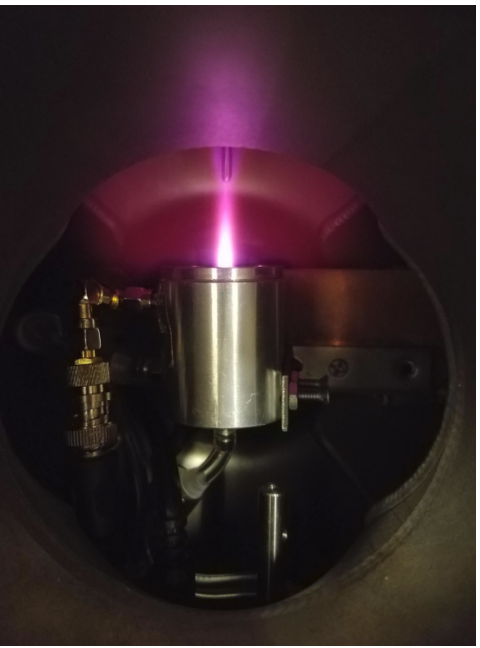

(b)

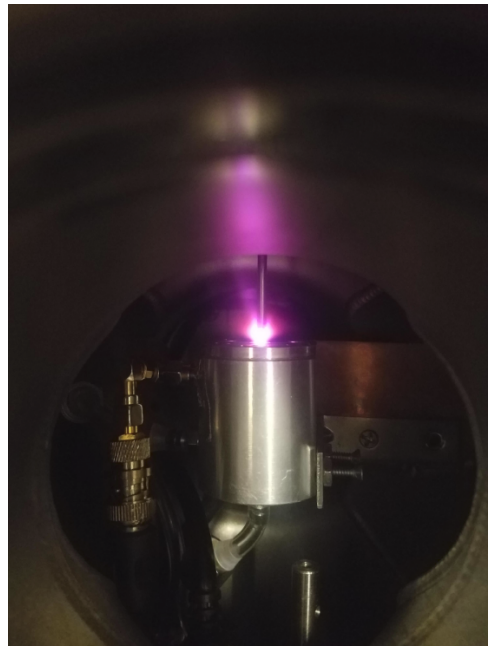

(c)

Figure 3.9: Orifice thruster configuration testing with pitot tube at various locations within the thruster plume.

If the pitot tube was misaligned with the flow when it was close to the thruster exit, plume deflections were easily seen, and the pitot was adjusted accordingly. However, moving downstream from 1-3 inches (0.0254-0.0762 m) away from the exit created an additional variability in pitot alignment, since the pitot tube began to disappear behind the vacuum chamber wall. This increase in variability can be seen as the increase in vertical error, as seen in Figure 3.8. In addition, horizontal error bars represent an estimated parallax error of $\pm 0.64 \mathrm{~mm}$. Initial alignment of the pitot tube required a zero reference point with the exact thruster exit plane so that the pitot tube could be axially translated precisely. It is possible that the difference between looking down through the top of the vacuum chamber view-port window, and 
determining the exact exit plane of the thruster, could have induced a conservative parallax error of up to $0.64 \mathrm{~mm}$ in translation.

The general trend in $\frac{P_{s}}{P_{0}}$ is as expected for both runs in Figure 3.8. Equation 2.6 indicates that a value of $\frac{P_{s}}{P_{0}} \approx 0.485$ will result in $M=1$. The trends in Figure 3.8 would then both indicate that Mach number was greater than 1 between $1.27 \mathrm{~mm}$ and $10 \mathrm{~mm}$ from the thruster exit. Past this location, the total pressure in the flow continues to increase, and flow velocity decreases.

Although the lower $3 \sigma$ bounds for the plasma operation runs indicate slightly lower pressure ratios than the cold gas tuns, and therefore an increase in exhaust velocity, the change is insignificant for this experiment. The observed pressure ratios still lie between the error bars of the cold gas operation. This is as expected, however, due to losses in the current thruster configuration and power system used for testing.

As the power supply used in the experiment outputs a constant power, the applied $\mathrm{RF}$ voltage is a function of the impedance of the physical device. This voltage was previously measured to be $199 \mathrm{~V}$ for this thruster in its current configuration [24]. Nominal RF voltage in previous experimentation for Pocket Rocket, which saw much larger increases in estimated exhaust velocities, was set for $240 \mathrm{~V}$ [1, 25]. Given the power losses in the current system, a much lower power density is being deposited into the plasma discharge tube. Therefore, this thruster is not operating at optimal conditions, and performance comparisons to previous experimentation cannot be made. The tested thruster in this work previously indicated plasma densities at the exit of the gas discharge tube to be approximately $n_{e}=7.5 \times 10^{16} m^{-3}$ [24]. Comparatively, the experimentally validated CFD results for Pocket Rocket in its nominal operation indicated plasma density at the thruster exit to be approximately $n_{e}=1 \times 10^{18} \mathrm{~m}^{-3}$, resulting in neutral heating upwards of $1000 \mathrm{~K}$ [1]. This is a further indication that the thruster tested in this work cannot be compared to previous Pocket Rocket ex- 
perimentation. However, the experimental results from the tested thruster can still confidently be used to validate the CFD model for full micro-nozzle flow characterization.

\subsubsection{MN-5 Nozzle Configuration Testing}

Given that the MN-5 nozzle would choke a smaller mass flow rate than the orifice configuration, a higher plenum pressure was selected to have an indicated pressure of 2.5 Torr. This correlates to an actual pressure of $6.1 \pm 0.5$ Torr, after the MKS correction factor is applied. For both cold gas and plasma operation runs, the ambient vacuum pressure sat at approximately $0.45 \pm 0.05$ Torr. The pressure ratio results

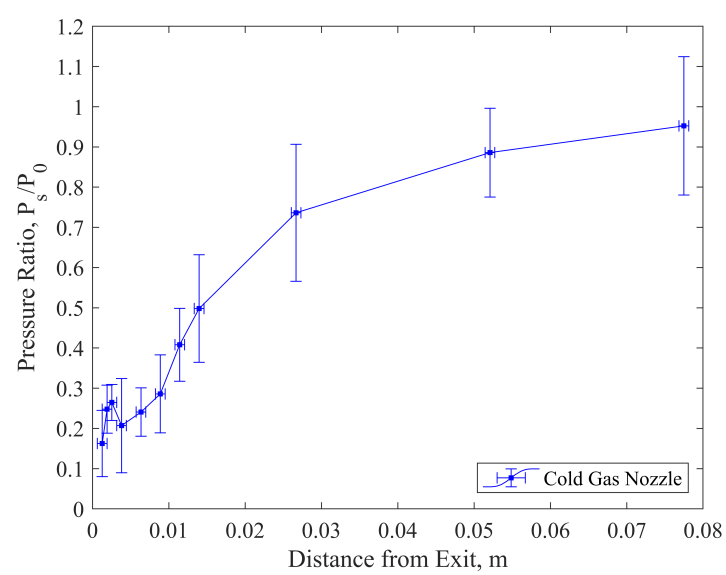

(a)

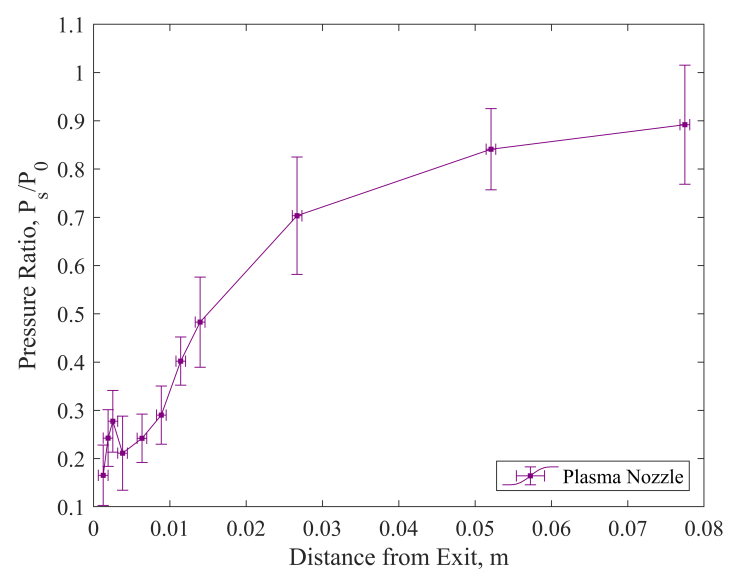

(b)

Figure 3.10: MN-5 nozzle experimental results for (a) cold gas testing, and (b) plasma operation testing $\left(P_{p l}=6.1 \pm 0.5\right.$ Torr, $P_{a}=0.45 \pm 0.05$ Torr $)$. Vertical error bars represent $3 \sigma$, horizontal error bars represent $0.64 \mathrm{~mm}$ of parallax error.

for both conditions can be seen in Figure 3.10. As compared to the orifice discharge tube configuration tests, the pressure ratio increases past the thruster exit at a much faster rate, with initial ratios of $\frac{P_{s}}{P_{0}}=0.15$. This in turn indicates a much faster flow velocity. Assuming a constant ratio of specific heat of $k=1.667$ for argon, the 0.15 
pressure ratio translates to an exit Mach number of approximately $M=1.85$ at a location of $1.27 \mathrm{~mm}$ past the thruster exit plane.

Upon testing the MN-5 nozzle configuration, shock diamonds were observed to be present in the flow. This is reflected in the pitot measurements in Figure 3.10 as a large spike in $\frac{P_{s}}{P_{0}}$ at $2.54 \mathrm{~mm}$ from the thruster exit. Figure 3.11 shows this region of interest for both test cases.

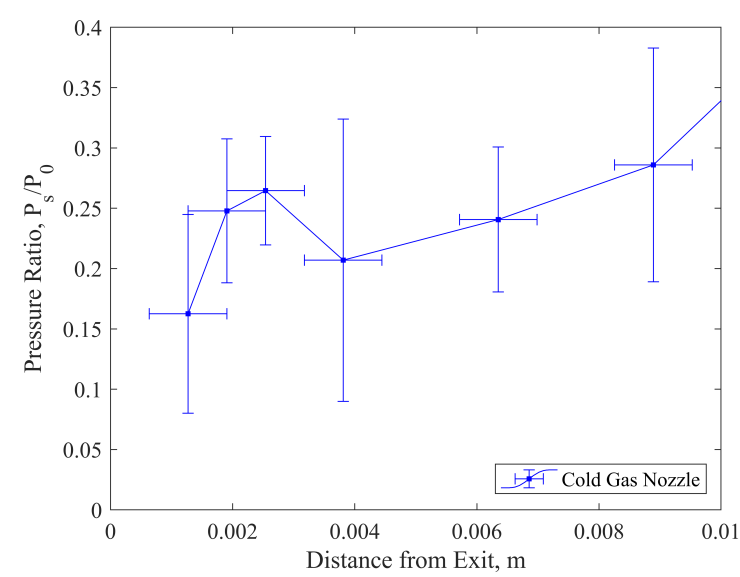

(a)

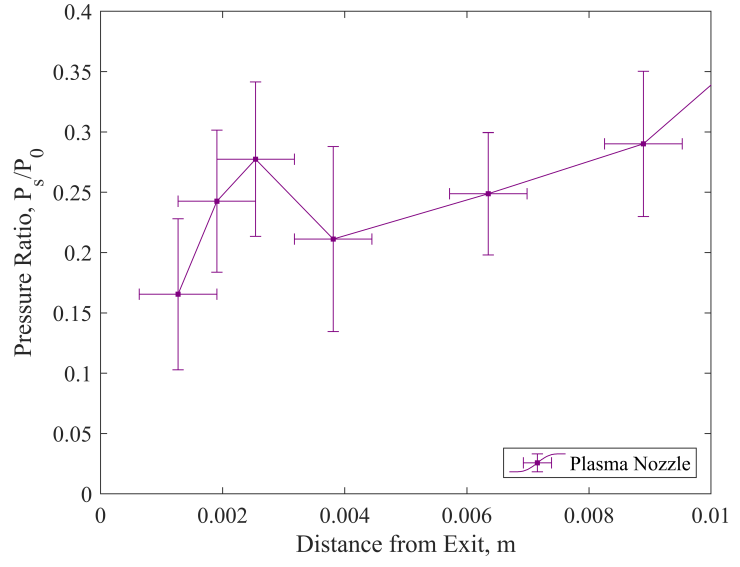

(b)

Figure 3.11: Zoomed in $\frac{P_{s}}{P_{0}}$ region of interest for MN-5 nozzle (a) cold gas testing and (b) plasma operation testing. Vertical error bars represent $3 \sigma$, horizontal error bars represent $0.64 \mathrm{~mm}$ of parallax error.

\subsubsection{Observed and Measured Nozzle Shock Diamonds}

The exhaust flow from each of the test cases has an exit pressure that is greater than the ambient vacuum pressure, leading to under-expanded flow. As a consequence, the exit pressure of the exhaust will initially encounter expansion fans at the nozzle exit, which will accelerate the flow and reduce the exit pressure in a Prandtl-Meyer supersonic expansion process [33]. In this expansion fan, the exit pressure is reduced until it is lower than the ambient pressure. However, a discontinuity exists at the center-line of the flow, and the expansion fan will reflect outwards off this boundary. 
In turn, a compression fan is created upon this reflection that acts to increase flow pressure once again. If the compression waves are strong enough, they can merge into oblique shocks. At the merged shock triple point, a bright region in the flow can appear that is known as a Mach, or shock diamond [34, 33]. Across a shock wave, gas properties change almost instantaneously in an increase of static pressure, temperature, and density [8]. This sudden change in gas properties can be seen in Figure 3.11 for both test cases, with a peak pressure ratio at $2.54 \mathrm{~mm}$. Without a shock present, the pressure ratio would gradually increase, as is the case from the orifice test plots in Figure 3.8.

Exhaust plume observation was used to build confidence in the determination that the spike in data shown in Figure 3.11 has indicated the presence of oblique shock waves. Figure 3.12 shows a picture of the thruster with a ruler at the exit,

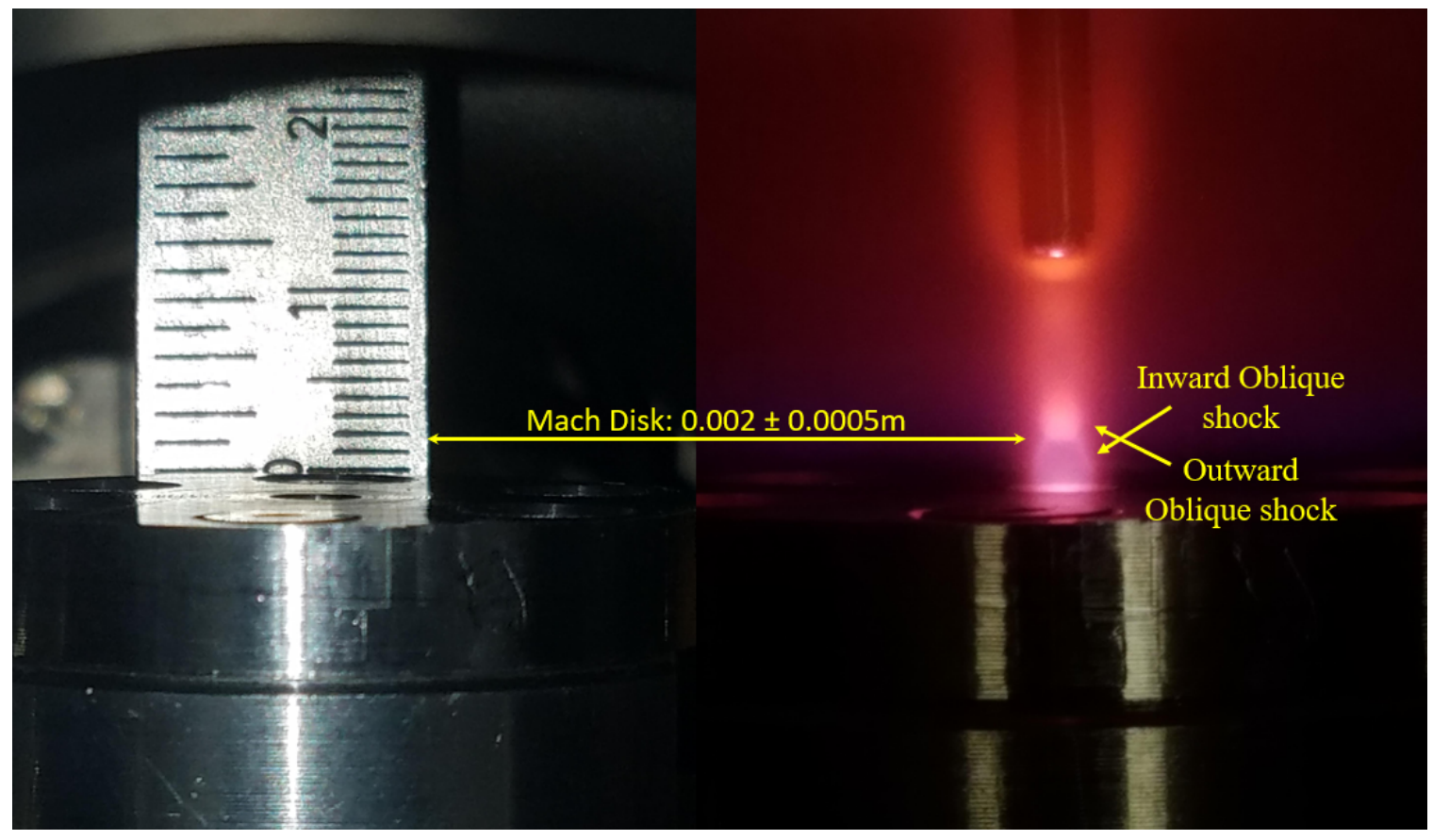

Figure 3.12: Approximate location of observed shock diamond during testing.

side-by-side with the observed standing shock diamond during testing. Both pictures used a camera set to a focal length of $4.2 \mathrm{~mm}$, from the surface of vacuum chamber 
view-port window, in order to recreate the same picture frame as closely as possible.

From Figure 3.12, the shock diamond in the flow was observed to sit at approximately $0.002 \pm 0.0005 \mathrm{~m}$, with surrounding inward and outward oblique shocks. This would indicate that the shock disk is located between the measured locations of $0.0019 \mathrm{~m}$ and $0.00254 \mathrm{~m}$, which is in good agreement with the collected pressure ratio data. The first data point at $0.00127 \mathrm{~m}$ in Figure 3.11 would exist within the nozzle expansion wave and experience an increase in velocity and decrease in static pressure. As seen in Figure 3.11, this results in the lowest recorded pressure ratio values. The next data point at a location of $0.0019 \mathrm{~m}$ would exist in the first inward shock location, before the shock diamond. At this location, static pressure, temperature, and density would spike, as indicated by the collected pressure ratio measurement. The next data point at $0.00254 \mathrm{~m}$ would exist within the second, outward oblique shock, and would again experience an increase in static pressure, temperature, and density. The flow would then return to an expansion wave, therefore increasing velocity and decreasing static pressure once again, at the $0.00381 \mathrm{~m}$ location.

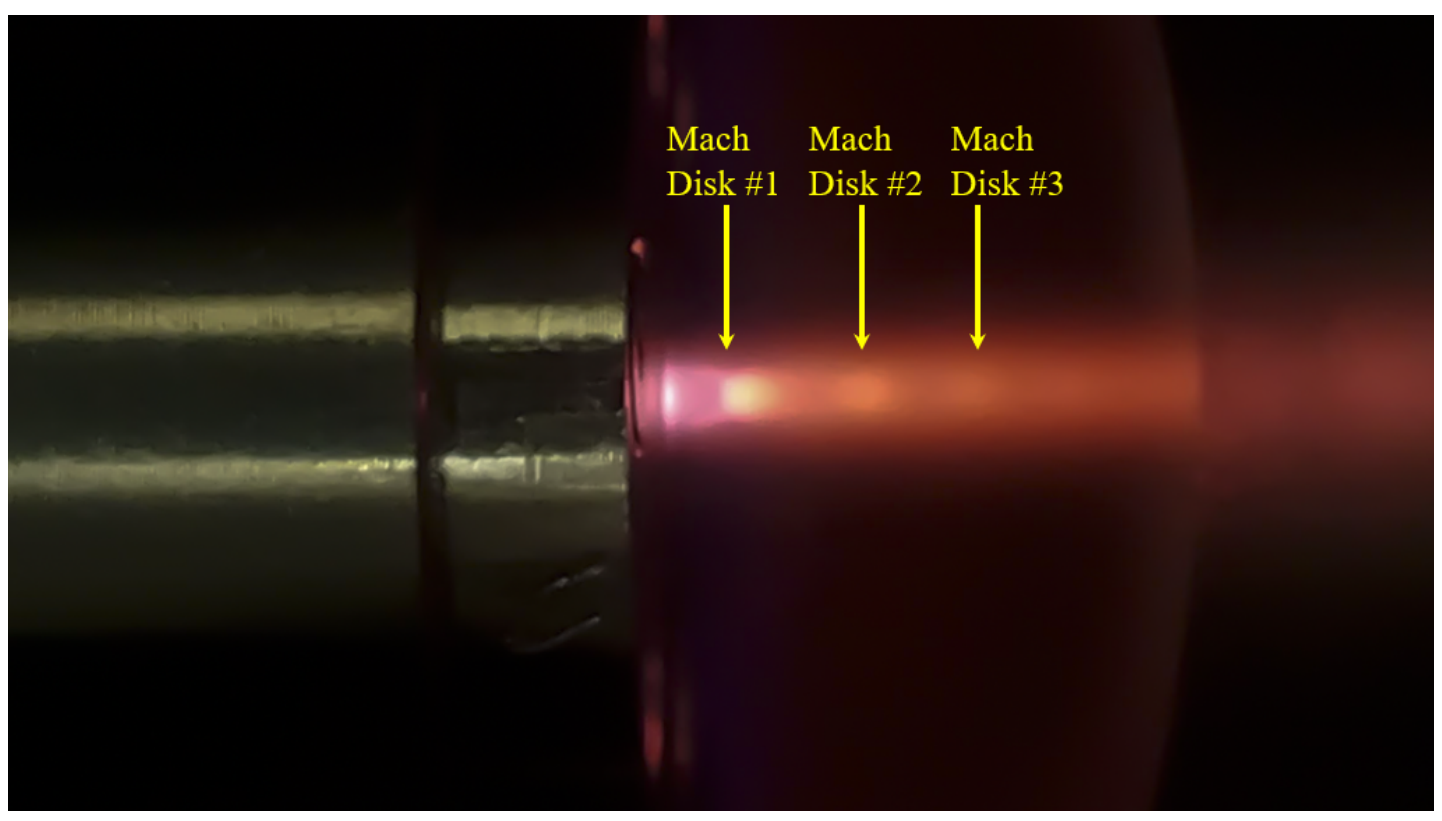

Figure 3.13: Nozzle test run with 3 observed shock diamonds. 
Additionally, in order to confirm that the pitot measurements were indeed picking up shock locations within the data, a single test run was conducted with the plenum pressure $P_{p l}$ set at $10 \pm 0.5$ Torr, with ambient vacuum conditions of $0.85 \pm 0.05$ Torr. This run induced 3 observable standing shock diamonds, as seen in Figure 3.13. The pitot tube was then placed at the observed locations before, in the middle of, and after the visible shock diamond. By assuming a constant ratio of specific heat for argon, the Mach number can be calculated for the pressure ratios obtained from the 10 Torr plenum pressure run. However, since the pitot tube is in supersonic flow, ideal isentropic equations cannot be used, as a bow shock will form across the pitot tip [35]. The bow shock from the pitot tube can be seen from the same image in Figure 3.12, and is directly pointed out in Figure 3.14.

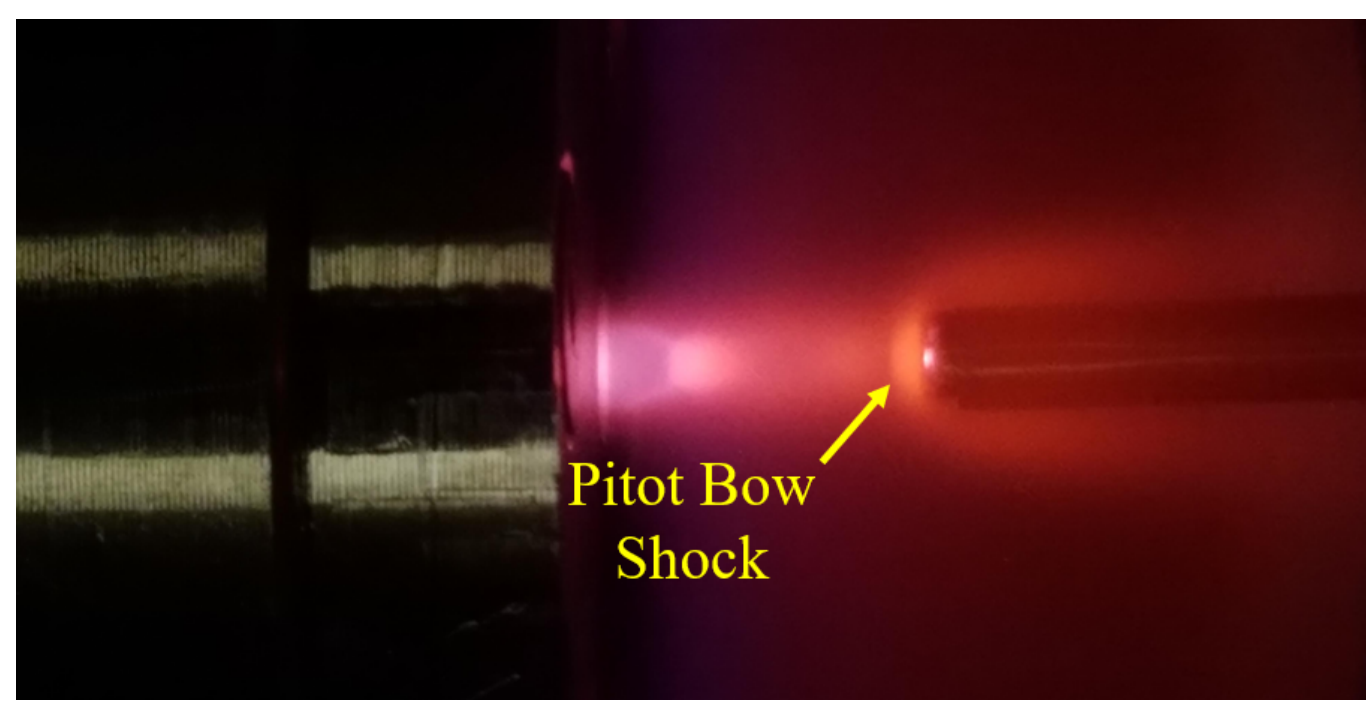

Figure 3.14: Pitot-static tube bow shock.

A schematic of the pitot tube bow shock can be seen in Figure 3.15. The bow shock forms along the sudden change in direction across the flow's streamlines, as a consequence of not being directly centered with the pitot tube. As streamlines pass through the center of flow and stagnate at the tip of the pitot tube, the fluid is first non-isentropically decelerated from a supersonic speed $V_{0}$ to a subsonic speed $V$, and then isentropically decelerated to zero at the stagnation point [35]. The streamlines 
are normal to the bow shock wave and create an additional total pressure loss, and static pressure increase across the shock. These are the pressures sensed by the pitotstatic tube [35].

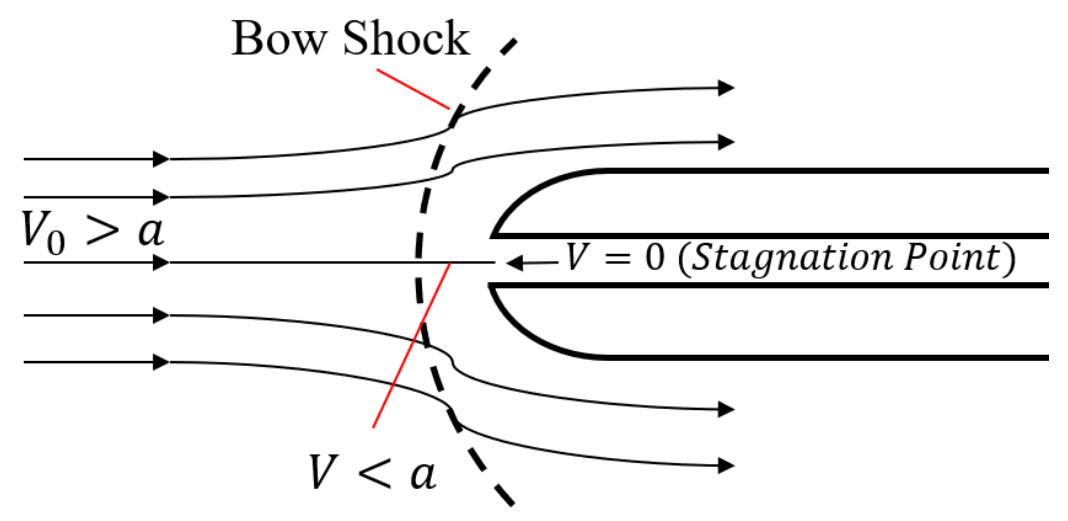

Figure 3.15: Bow shock diagram.

If $P_{01}, P_{1}$ and $M_{1}$ are the supersonic stagnation, static, and Mach conditions before the flow traverses the pitot bow shock, and $P_{02}$ is the stagnation condition past the bow shock, then $M_{1}$ can be solved as Rayleighs supersonic pitot tube equation [35]

$$
\frac{P_{02}}{P_{1}}=\frac{\left[\frac{k+1}{2} M_{1}^{2}\right]^{k /(k-1)}}{\left[\frac{2 k M_{1}^{2}-(k-1)}{k+1}\right]^{1 /(k-1)}}
$$

Once again, the experiment pressure ratio data is in good agreement with the shock diamond observations. In the Mach vs. Distance plot in Figure 3.16, 3 shock locations are easily visible in the data. Surrounding each shock location in the data is an expansion wave followed by a compression wave. The expansion wave can once again be seen to decrease pressure, thereby increasing velocity and Mach number just before each oblique shock location. Next, a compression wave increases static pressure and temperature, thereby decreasing velocity and Mach number [34, 33]. This process is repeated for a total 3 times, matching the observed 3 shock diamonds. 


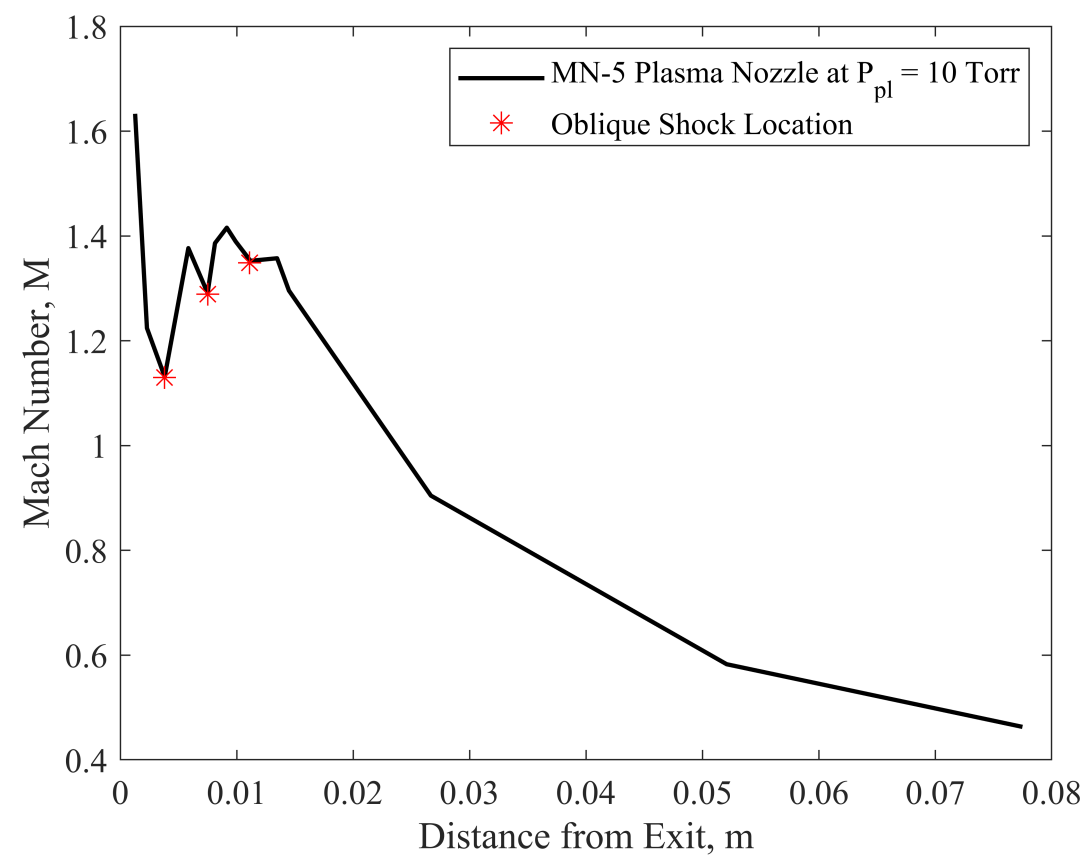

Figure 3.16: Mach number plot with 3 observed shock locations.

It is possible that additional, very faint shocks may have existed after the third shock location. However, data was only collected near the clearly visible shock diamonds in the flow.

The direct measurement of shocks within Pocket Rocket's exhaust flow is a very good indication that the pitot-static tube and selected instrumentation are sensitive enough to fully characterize the flow; both in terms of detecting discontinuities in the flow, and in terms of successfully characterizing regions with no discontinuities. In particular, the ability to sense refined shock regions in the flow, clearly defined within $3 \sigma$ error bars, is a good indication that the pitot diameter is of an appropriate size in relation to the diameter of the exhaust jet. Inaccurate and highly fluctuating flow measurements can occur in pitot tubes with diameters that are much larger than the diameter of the exhaust jet [36]. Additionally, the detection of small yet rapidly changing pressure measurements across flow discontinuities further provides confidence in the utilization of the convectron gauges as a low cost option that retains 
high resolution. This is important as pitot-static tubes may typically be used in conjunction with either pressure transducers or capacitance manometers, along with an accompanying increase in experiment cost.

The ability to characterize shock structures is also especially important to verify when using the experimental flow field results to validate the CFD model. The locations and sizes of measured oblique shocks within the thruster flow can now be compared to the shock structures predicted by the CFD simulations. 


\section{Chapter 4}

\section{COMPUTATIONAL FLUID DYNAMICS}

Fabricating and testing every nozzle design, in order to optimize Pocket Rocket performance, would not only be time consuming, but also very costly. This would especially be true for testing various unique nozzle geometries with complicated bell-shaped contours. These contours, beyond the simple conical shape, would likely require advanced and costly methods of fabrication; including electrostatic discharge, metal additive manufacturing, or femtosecond laser machining [37]. Instead, the testing completed for both the orifice and MN-5 nozzle geometry can be used to validate CFD efforts. This significantly reduces both the time it would take to find an optimized design, and the costs associated with experimentation. By validating the experimental test cases, confidence can be transferred to the CFD-ACE+ software. The validation of the CFD simulations will allow this work to simulate the 9 nozzle geometries with various nozzle half angles and area ratios from section 2.2. CFD validation will also allow for future work to quickly find any one exact optimized nozzle for a Pocket Rocket mission.

\subsection{Software: CFD-ACE+}

Several previous studies have now used the software suite CFD-ACE + to successfully verify and validate Pocket Rocket models with experimental results $[1,24]$. CFD$\mathrm{ACE}+$ remains one of the few commercially available tools can accurately model the required plasma physics of Pocket Rocket, in order to fully capture thruster behavior $[25]$.

CFD-ACE + uses the finite volume method to solve the conservation of mass, momentum, and energy equations. The software package can also solve scalar transport 
equations and chemical species reactions, which enable fluid simulations to be coupled with thermal, chemical, and electrical components.

In order to validate the CFD with experimental results, steady state heat transfer simulations will be conducted. Transient plasma 2D CCP simulations can be performed, but can take up to 8 hours to run $150 \mathrm{~ms}$ of simulation time [1]. This simulation time is not long enough for Pocket Rocket to reach a steady thermal state with respect to wall heating effects. Furthermore, the computational time required to do so, between 10-100 seconds, is not practical. Therefore, the 2D, axisymmetric simulation domain in Figure 4.1 has been developed to encompass neutral gas flow and heat transfer in order to model the thruster's wall heating effects. Each steady state simulation can be run on the order of minutes, rather than hours.

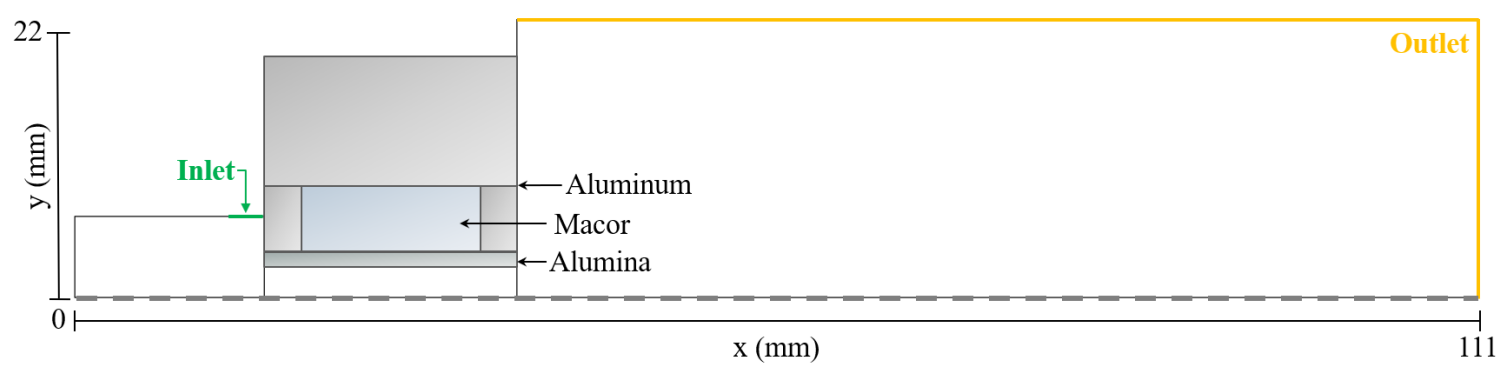

Figure 4.1: Full simulation domain for thruster with the orifice discharge tube geometry.

The full simulation domain consists of the thruster inlet, leading into the plenum chamber, alumina gas discharge tube, and downstream expansion region with an outlet boundary condition. Surrounding the alumina discharge tube is the thruster housing components consisting of the Macor cylinder and grounded aluminum housing. The typical metal copper electrode used for Pocket Rocket was omitted as the tested thruster used a very thin layer of copper tape as the electrode instead. This extremely thin layer would insignificantly change the results of the heat transfer simulation. 
This 2D domain is axisymmetric about the x-axis of the model, and has been created to replicate the exact dimensions of the tested thruster. The only difference between the orifice configuration and the nozzle domain configuration is the addition of the aluminum nozzle piece, located at the far right of the discharge tube, as seen in Figure 4.2.

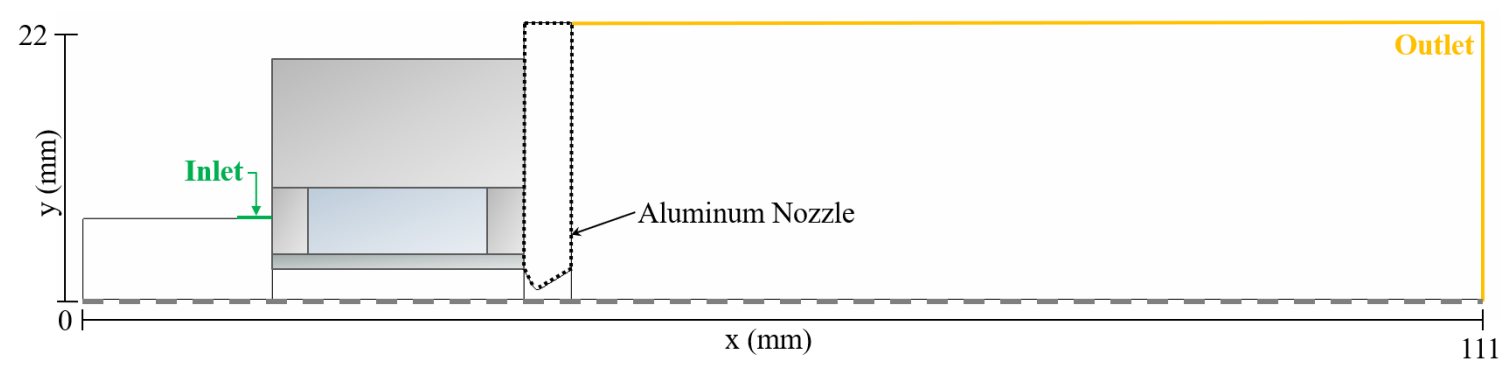

Figure 4.2: Full simulation domain for thruster with the MN-5 nozzle geometry.

The approximate plasma heating effects can be modeled in a steady state simulation by applying constant volumetric and surface power densities to the inside of the discharge tube volume and the surface of the alumina tube. As previous work has verified, this effectively models the heating effects due to ion neutral collisions within the discharge tube and ion bombardment to the alumina walls [1].

By applying the transient simulation plasma conditions, previously validated experimentally [24], the spatially averaged power density can be determined for the thruster used in this works' testing. A carpet plot of phased averaged power density within the discharge tube can be seen in Figure 4.3.

As expected, the peak power density occurs in the center of the discharge tube at the RF electrode [1]. From Figure 4.3, the spatially averaged volumetric power density is $1.33 \times 10^{6} \mathrm{Wm}^{-3}$. This applied power density is approximately $78 \%$ lower than previous Pocket Rocket experimentally validated studies, which indicated an average volumetric power density of $5.8 \times 10^{6} \mathrm{Wm}^{-3}[1]$. This large decrease in power density further confirms that the thruster used in testing for this work will exper- 


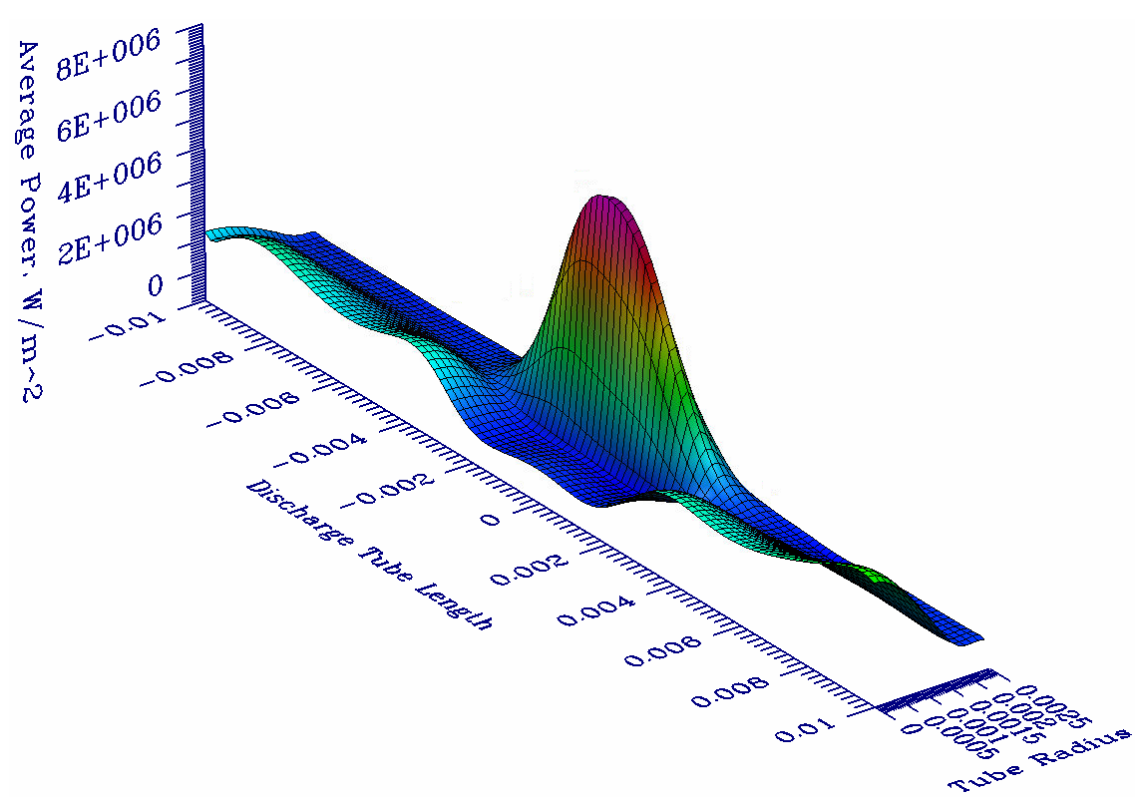

Figure 4.3: Carpet plot of average power density within the gas discharge tube.

imentally show a correlated large decrease in propellant heating and performance. Assuming the previously validated $1.33 \times 10^{6} \mathrm{Wm}^{-3}$ power density for this work is applied throughout the entire discharge tube, a total of $0.48 \mathrm{~W}$ can be applied to the discharge tube fluid volume to model the volumetric heating effects in the steady state simulations. In order to determine the wall heating effects due to ion bombardment to the thruster walls, the previously validated transient model conditions can once again be used [24]. Figure 4.4 shows the transient model's plot of average ion current density along the length of the alumina tube. By assuming the full $199 \mathrm{~V}$ of applied power reaches the copper electrode, this current density can be used to estimate surface power density along the length of the discharge tube. The spatially averaged current density is $4 \mathrm{~mA} / \mathrm{cm}^{2}$, which correlates to a surface power density $8000 \mathrm{~W} / \mathrm{m}^{2}$. This was used to simulate ion bombardment wall heating effects in the steady state simulations.

For all steady state simulations conducted in this study, the modules of Flow, Heat Transfer, Turbulence, and Chemistry/Mixing were used in the CFD-ACE+ software 


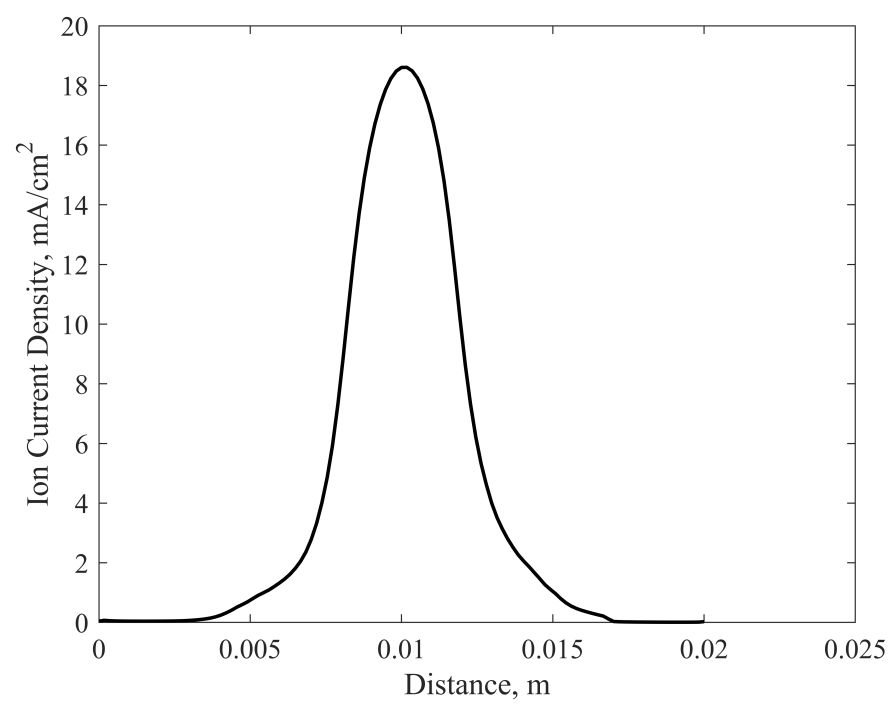

Figure 4.4: Ion current density within the gas discharge tube.

suite. Turbulence was set to the standard value of 0.9 for the turbulent Prandtl and Schmidt number. Each fluid volume condition used the ideal gas law to solve for density and Mix Sutherland's Law to solve for viscosity. Thermal conditions were set to replicate a constant specific heat of $C_{p}=520 \mathrm{~J} / \mathrm{kgK}$ with thermal conductivity represented with a constant Prandtl number of 0.66 for pure argon gas. Table 4.1 outlines the thermal properties of the thruster housing materials used for each steady state simulation.

Table 4.1: Thruster housing thermal properties with density $\rho$, specific heat $c_{p}$, and thermal conductivity $k$.

\begin{tabular}{llll}
\hline & $\mathrm{Al}_{2} \mathrm{O}_{3}$ & Macor & $\mathrm{Al}$ \\
\hline$\rho\left[\mathrm{kg} / \mathrm{m}^{3}\right]$ & 3950 & 2520 & 2700 \\
$c_{p}[\mathrm{~J} / \mathrm{kg}-\mathrm{K}]$ & 880 & 790 & 900 \\
$k[\mathrm{~W} / \mathrm{m}-\mathrm{K}]$ & 40 & 1.46 & 210 \\
\hline
\end{tabular}

For all simulations developed to model the heating effects of Pocket Rocket, the constant volumetric heat addition of $1.33 \times 10^{6} \mathrm{Wm}^{-3}$ was applied to the fluid volume 
of the gas discharge tube. Surface heat addition was modeled with the surface power density addition of $8000 \mathrm{~W} / \mathrm{m}^{2}$ to the entire alumina tube's inner surface.

Inlet and outlet boundary conditions varied between the orifice configuration and MN-5 nozzle configuration. After applying the correction factors for argon gas to the indicated convectron gauge readings during testing, it was determined that the orifice testing was conducted with a plenum pressure set to a true pressure of $2.9 \pm$ 0.5 Torr, while the ambient vacuum pressure sat at $0.9 \pm 0.05$ Torr. Additionally, the true plenum pressure for the MN-5 nozzle testing was set to $6.1 \pm 0.5$ Torr, with an ambient downstream pressure of $0.45 \pm 0.05$ Torr. An error of 0.5 Torr in the plenum of the thruster can significantly influence downstream exhaust parameters. Due to this large error, CFD simulations were conducted with the upper and lower bounds on plenum pressure. For each simulation test case, the upper and lower bounds on plenum pressure was applied to the inlet condition in the simulation domain, while the outlet condition was set to the upper and lower bounds of the ambient vacuum conditions observed during testing.

\subsubsection{CFD Validation Mesh}

The structured mesh used for the full simulation domain for the MN-5 nozzle can be seen in Figure 4.5. The only difference in mesh creation for the orifice discharge tube configuration is the removal of the nozzle, located at the end of the discharge tube.

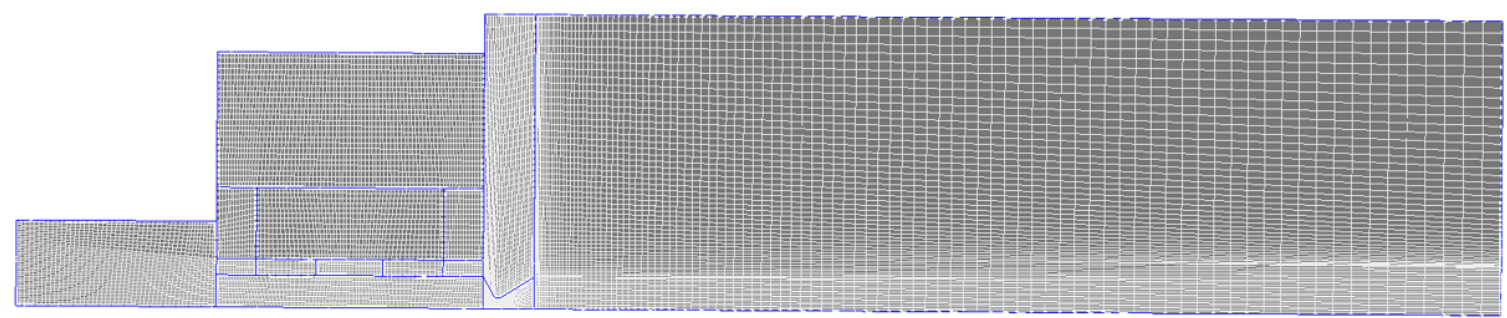

Figure 4.5: Full MN-5 nozzle simulation domain mesh. 
The regions with the highest simulation sensitivity include the discharge tube, nozzle, and downstream expansion region along where the thruster plume will be directed $[1,24]$. The mesh has been defined to have a higher number of cells within these regions, with a lower number of cells in the upper expansion region, where flow parameters will not be studied.

A mesh convergence study was preformed for the orifice configuration's fluid domain in order to determine the most accurate mesh density range. The selection of the most optimal simulation mesh typically consists of the density with the least amount of cells, in which the flow variables are no longer affected by cell count. By selecting the least amount of cells, computation time can be saved, while retaining a high degree of simulation accuracy. This convergence study was performed with only the flow module in CFD-ACE+, with increasing mesh densities of 739, 1634, 7467, 12882, and 88472 cells in the orifice configuration fluid domain. All fluid domain parameters were used as previously described, with standard operating inlet pressures of $200 \mathrm{~Pa}$ and downstream outlet pressures of $50 \mathrm{~Pa}$. As the flow characterization of the Pocket Rocket exhaust plume is of interest to this work, parameters to determine mesh convergence were taken as the local maximum within the domain of the downstream region, just after the discharge tube exit. Figure 4.6 shows the global maximum values within the downstream fluid domain for velocity, static pressure, and total pressure.

Figure 4.6 shows that the variables of interest are within $1 \%$ difference between the mesh density of 12882 and 88472 cells. The mesh with a density of 12882 cells in the fluid and alumina tube domain was chosen in order to save computation time. The solid alumina tube domain used the same mesh density scheme as the fluid domain in order to accurately simulate heat transfer between the alumina tube and the fluid flow in the discharge tube. The fluid domain mesh density is in good agreement with previous work, which found a converged mesh density of 11075 cells for a similar 


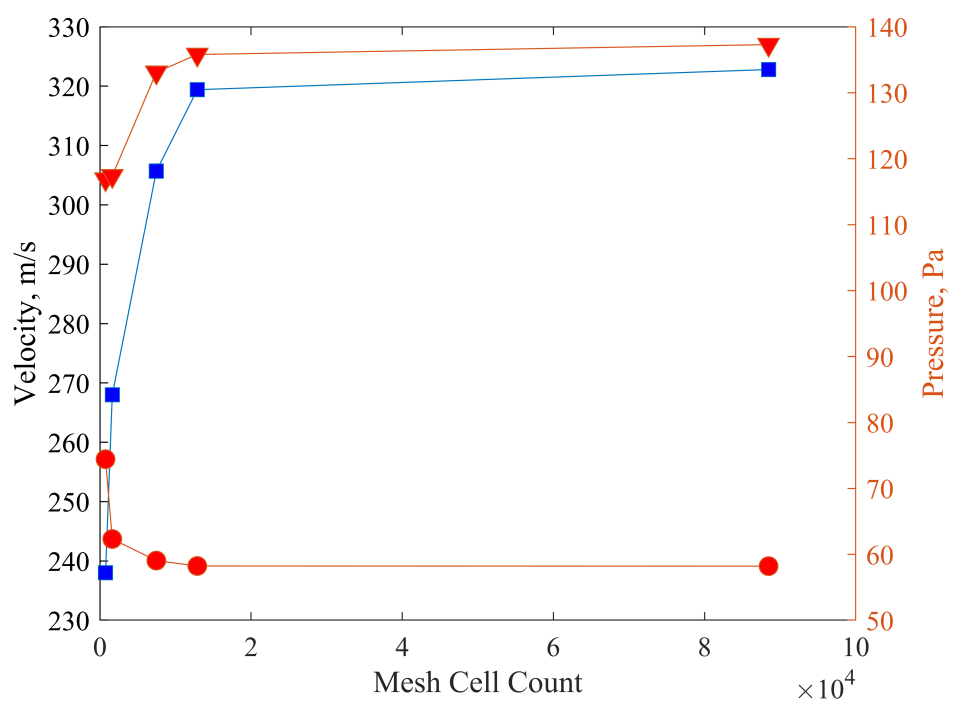

Figure 4.6: Mesh convergence results for velocity (blue squares), static pressure (red circles), and total pressure (red triangles).

Pocket Rocket fluid domain [1]. Given the converged mesh density of 12882 cells for the orifice configuration fluid domain, the same density scheme in the structured mesh was applied to the nozzle configuration fluid domain. The MN-5 nozzle fluid domain was found to add an additional 1092 cells. The housing components for both simulation domains consisted of an additional 2964 cells.

\subsubsection{Preliminary CFD Temperature and Pressure}

Figure 4.7 shows the steady state temperature results for both volumetric and surface heat additions. The model shows the orifice geometry at the top, which was ran at pressures of 3.4 Torr and 0.9 Torr in the plenum and downstream regions, respectively. The bottom model shows the MN-5 nozzle geometry, which had applied pressures of 6.6 Torr and 0.45 Torr in the plenum and downstream regions, respectively.

Given the applied volumetric and surface power densities that model the experimental thruster, it is clear that the CFD model is not predicting typical volumetric heating upwards of $1000 \mathrm{~K}$, as seen in other Pocket Rocket variations [1, 25]. A 

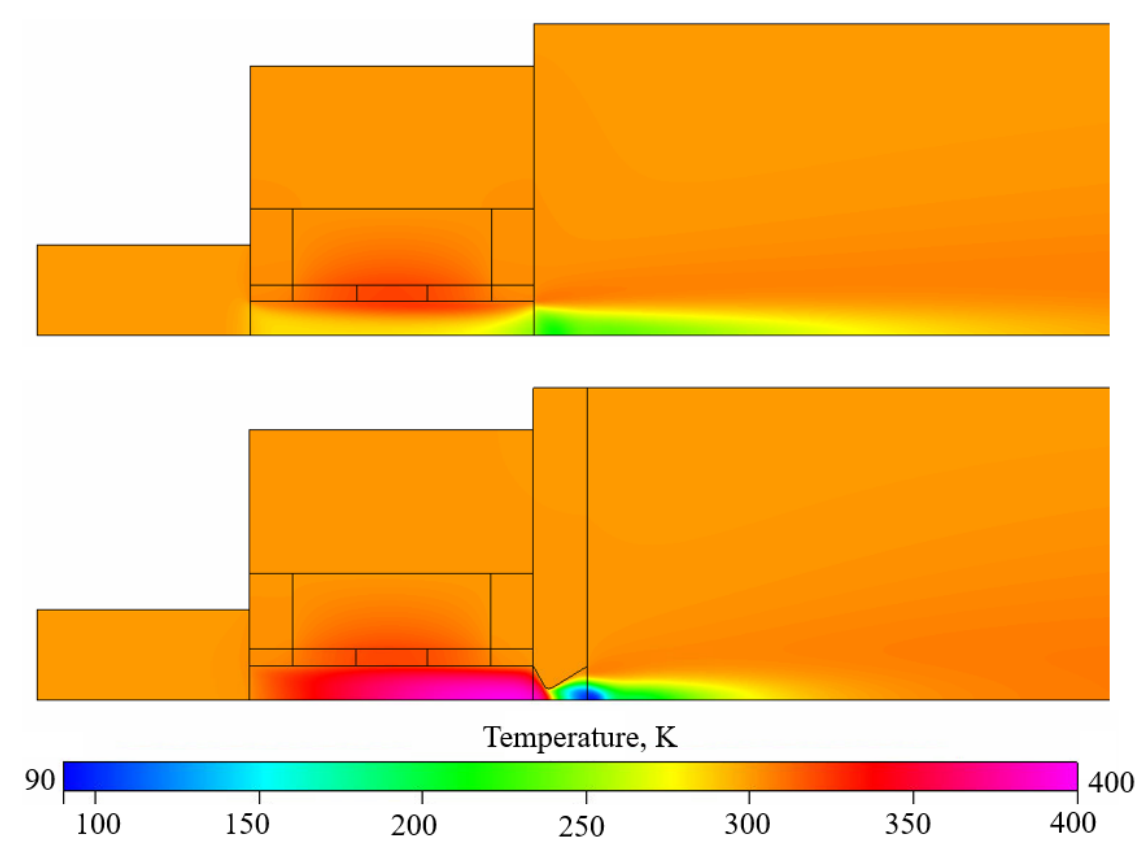

Figure 4.7: Simulation temperature results for (top) orifice configuration at $P_{p l}=3.4$ Torr, $P_{a}=0.9$ Torr and (bottom) MN-5 nozzle configuration at $P_{p l}=6.6$ Torr, $P_{a}=0.45$ Torr.

higher neutral gas heating occurs in the nozzle geometry simulation, as the pressure gradient in the gas discharge tube shifts further downstream to fill only the nozzle throat. This can best be seen in the static pressure model for the orifice geometry and

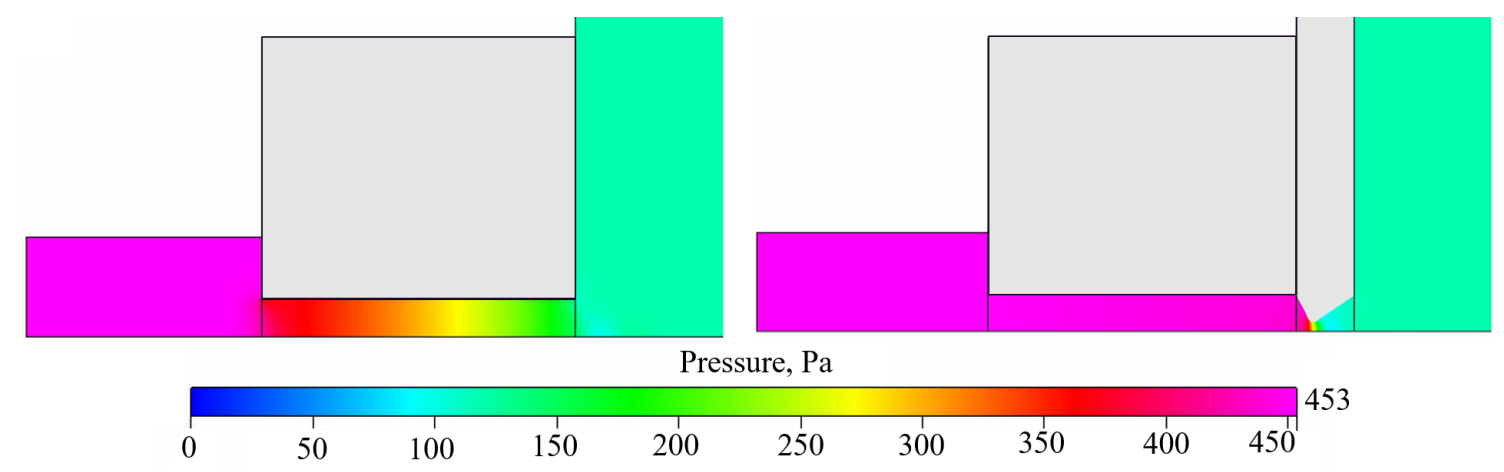

Figure 4.8: Simulation static pressure results for (left) orifice configuration at $P_{p l}=3.4$ Torr, $P_{a}=0.9$ Torr and (right) MN-5 nozzle configuration at $P_{p l}=3.4$ Torr, $P_{a}=0.9$ Torr.

MN-5 nozzle geometry in Figure 4.8. In order to make a direct comparison between the shift in pressure gradient, both simulations in Figure 4.8 were run with a plenum 
pressure of 3.4 Torr and a downstream pressure of 0.9 Torr.

In the orifice configuration, the pressure gradient starts at the discharge tube inlet, and gas begins to expand and cool throughout the tube, thereby lowering neutral gas heating. However, when the MN-5 nozzle is added, the pressure in the discharge tube matches that of the inlet, acting as an extension of the plenum. The pressure gradient to vacuum now shifts to a location just before the nozzle throat. By not allowing the gas to expand through the discharge tube, the overall neutral gas heating is increased in the discharge tube with the adddtion of a nozzle. In order to gain confidence in these initial results, the CFD must be validated with the experimental results.

\subsection{CFD Validation}

\subsubsection{Orifice Configuration}

Figure 4.9 shows the pressure ratio plots for the orifice configuration experimental results with the CFD (Average) simulation run at $P_{p l}=2.9$ Torr, and $P_{a}=0.9$ Torr. The upper and lower CFD bounds were run with $P_{p l}=2.9 \pm 0.5$ Torr, and $P_{a}=0.9 \pm 0.5$ Torr, respectively. Although there is a large error associated with the gas correction factor in the thruster plenum, and thus a large range of CFD bounds, the experimental results fit very nicely within the indicated bounds. Figure $4.9 \mathrm{~b}$ shows the orifice plasma testing results, and can be seen to match the CFD simulation almost directly through the average test values. Despite the large error in the system, confidence can be placed within the CFD simulations for the orifice geometry, for both cold gas and simulated steady state plasma heating effects. 


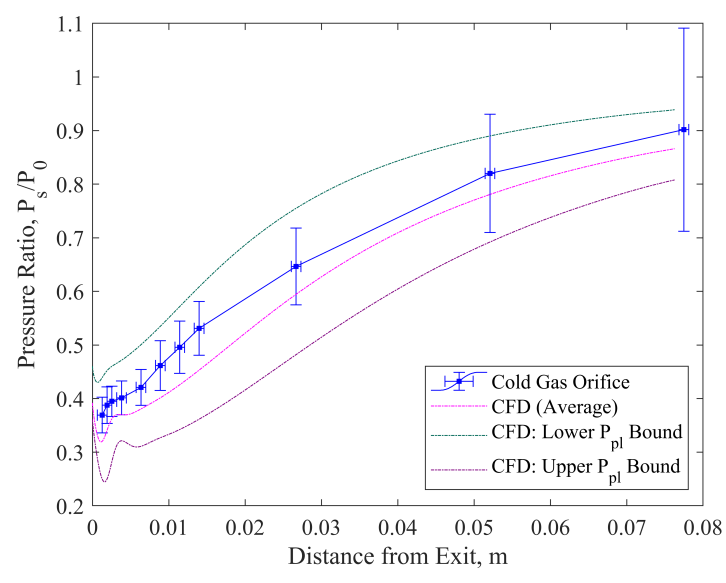

(a)

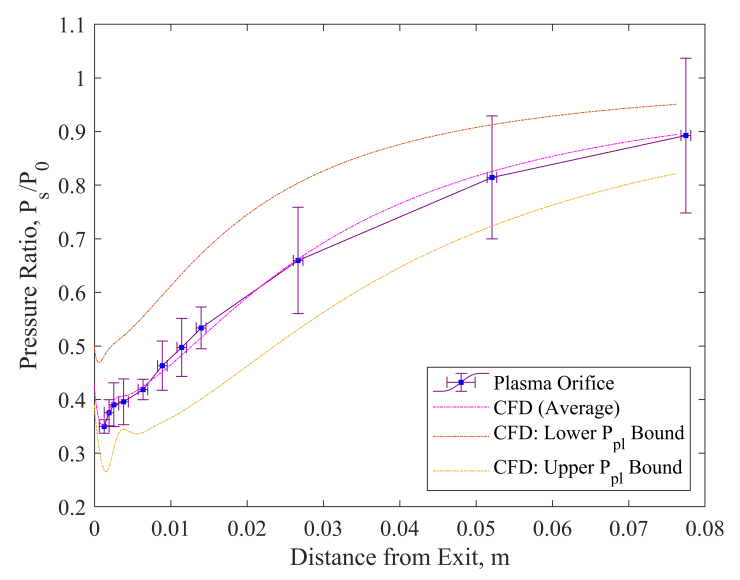

(b)

Figure 4.9: CFD validation results for the orifice thruster configuration during (a) cold gas operation and (b) plasma operation. The pink CFD (Average) results represent the simulation run at $P_{p l}=2.9$ Torr, $P_{a}=0.9$ Torr. The upper and lower CFD bounds were run with $P_{p l}=2.9 \pm 0.5$ Torr and $P_{a}=0.9 \pm 0.5$ Torr, respectively.

\subsubsection{MN-5 Nozzle Configuration}

The CFD upper bound for the MN-5 nozzle geometry consisted of plenum and downstream pressures of 6.6 Torr and 0.5 Torr, respectively. Simulations for the lower bound were run at plenum and downstream pressures of 5.6 Torr and 0.4 Torr, respectively. The CFD and experimental results for the cold gas runs can be seen in Figure 4.10 and results for the plasma operation runs can be seen in Figure 4.11.

Both the cold gas and plasma pressure ratios show similar trends when compared to their respective CFD bounds. Although the experimental averages no longer lie directly in between the CFD simulation bounds, as was the case for the discharge tube configuration, the upper $3 \sigma$ error bars from the experiment still lie within the bounds. In general, the experimental results can be seen to closely revolve around the upper bounds of each CFD simulation. It is possible that the CFD is showing slight error in downstream plume measurements. As the density and Knudsen number of 


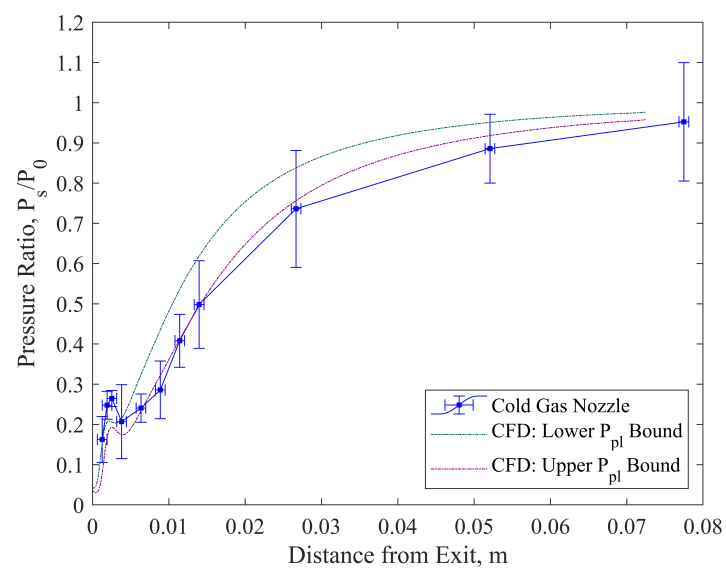

(a)

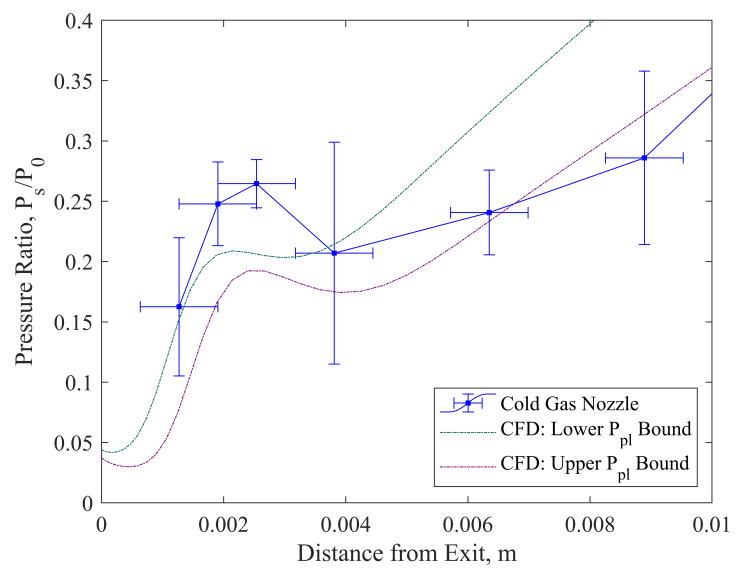

(b)

Figure 4.10: Cold gas CFD validation results for the MN-5 nozzle thruster configuration, showing (a) full measurement profile and (b) zoomed in profile, just after the thruster exit. The upper and lower CFD bounds were run with $P_{p l}=6.1 \pm 0.5$ Torr and $P_{a}=0.45 \pm 0.5$ Torr, respectively.

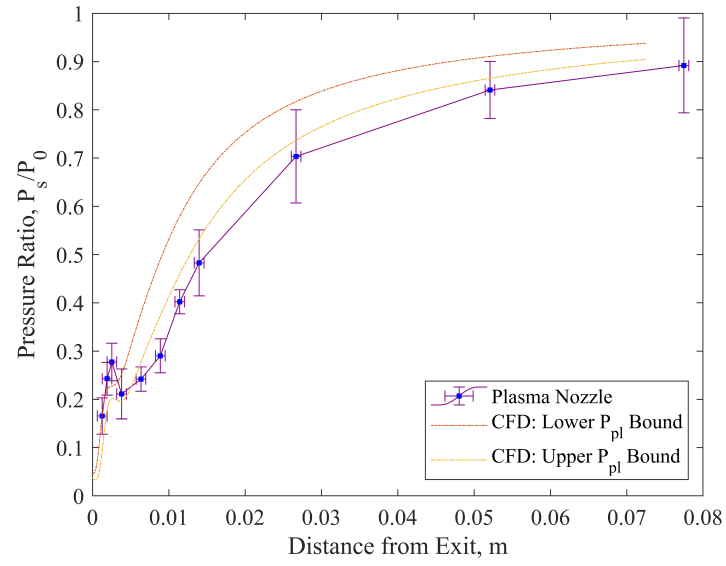

(a)

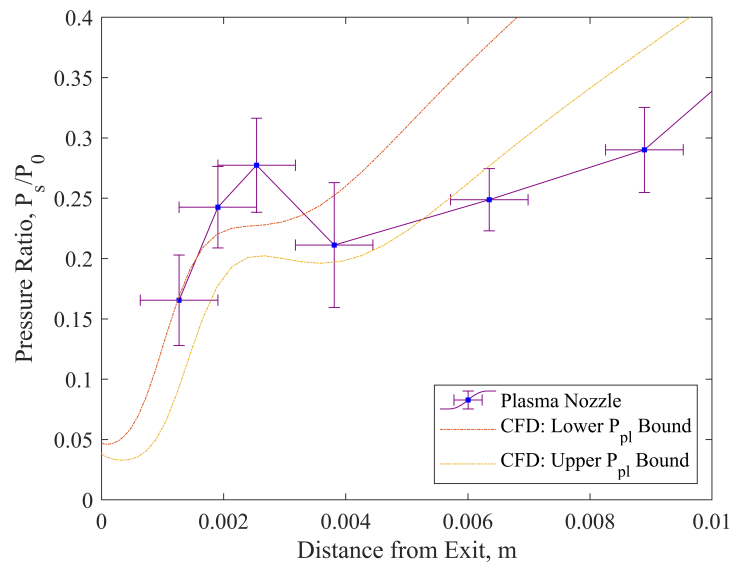

(b)

Figure 4.11: Plasma operation CFD validation results for the MN-5 nozzle thruster configuration, showing (a) full measurement profile and (b) zoomed in profile, just after the thruster exit. The upper and lower CFD bounds were run with $P_{p l}=6.1 \pm 0.5$ Torr and $P_{a}=0.45 \pm 0.5$ Torr, respectively.

the flow decrease downstream of the nozzle choke point, the flow will move closer to a molecular flow regime. Therefore, the the continuum flow mechanics of CFD-ACE+ for flow characterization cannot be fully guaranteed for large distances beyond the 
nozzle choke point [25]. It is also possible that the experimental results did in fact mimic the same operating conditions as the upper CFD bounds, and that the average convectron gauge gas correction factor has been underestimated from Appendix A Figure A.1 for nozzle testing. However, as the error bars of the experimental results still lie in between the expected CFD bounds, confidence can be placed in the CFD results for the nozzle configuration.

The two data points at $0.0019 \mathrm{~m}$ and $0.00254 \mathrm{~m}$ lie directly above both CFD bounds for both cold gas and plasma nozzle operation conditions. These two points were previously determined to lie in the oblique shock regions surrounding a standing shock diamond in section 3.3.1. This shock structure can also be seen in the CFD static pressure results in Figure 4.12, for the simulated heating simulation with the MN-5 nozzle. The CFD domain has been cropped to show the region of interest at
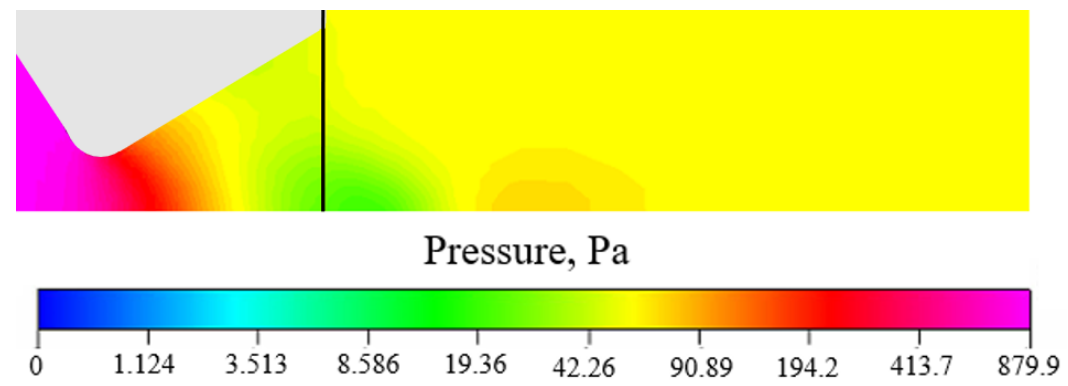

Figure 4.12: Zoomed in CFD static pressure profile through the MN-5 nozzle $\left(P_{p l}=6.6\right.$ Torr, $P_{a}=0.5$ Torr $)$.

the nozzle exit, showing a sharp increase in static pressure, just after the pressure has dropped through the diverging nozzle section. The downstream region just past this MN-5 nozzle exit plane also indicates strong shock regions in the Mach number plot in Figure 4.13. Mach number sharply increases at $0.0026 \mathrm{~m}$ from the thruster exit due to the static pressure increase and total pressure loss across the shock structures.

As seen in both Figures 4.10b and 4.11b, the data points at $0.0019 \mathrm{~m}$ and 0.00254 $\mathrm{m}$ are estimating a larger increase in pressure ratio than anywhere else in the flow. 


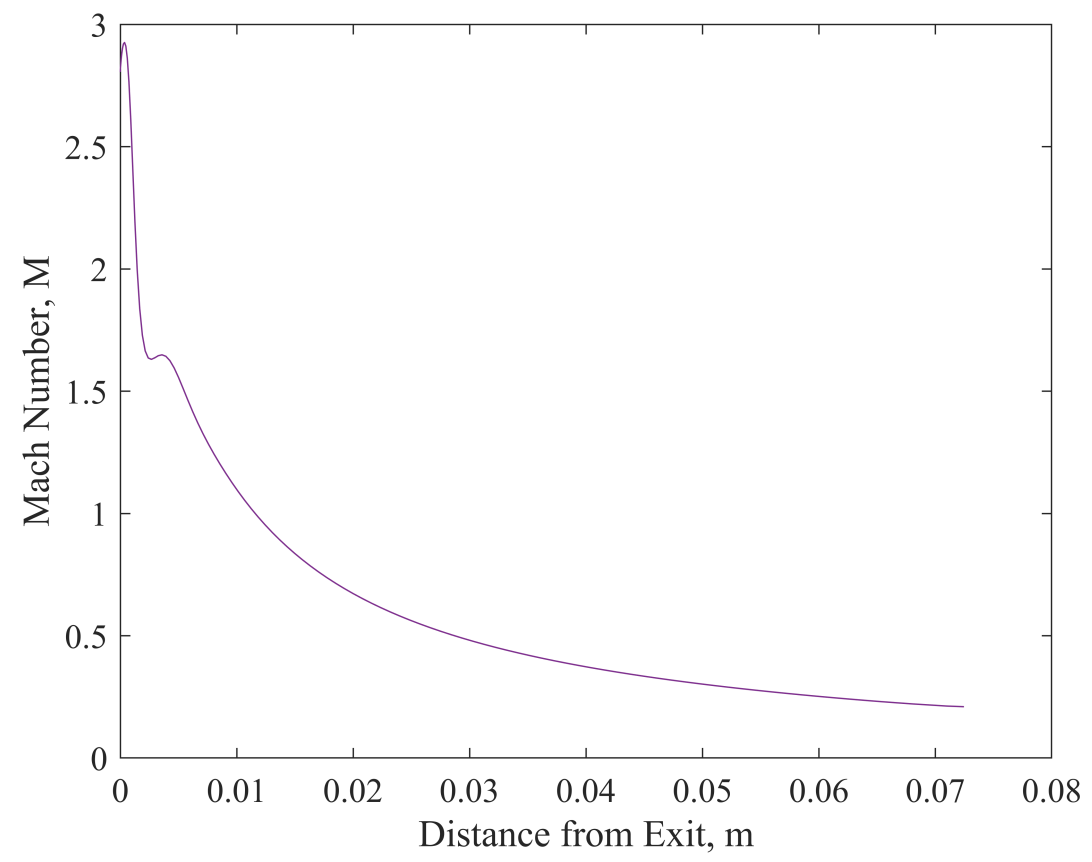

Figure 4.13: CFD Mach number plot showing downstream shock region after the MN-5 nozzle exit $\left(P_{p l}=6.6\right.$ Torr, $P_{a}=0.5$ Torr $)$.

One possible explanation for this is due to the shock-shock interaction that occurs between the pitot-static tube's bow shock and the oblique shocks within the flow. Due to the impinging shock-shock interaction, an additional increase in static pressure and temperature can occur, resulting in additional losses in total pressure [38]. In turn, this would lead to the over-prediction of $\frac{P_{s}}{P_{0}}$ within the oblique shock locations in the flow.

Despite the over-predictions in pressure ratio sensed by the pitot-static tube in supersonic flow, and the possible under-prediction of pressure ratio in the downstream CFD simulations, confidence can still be placed in the CFD results as the experimental error of $3 \sigma$ still lies between the expected CFD bounds. The orifice discharge tube configuration, which used a much lower plenum pressure, can also be fully validated as the experimental results lie between the CFD bounds with a $3 \sigma$ error. 


\subsection{Micro-Nozzle Design Simulation Results}

When integrating any propulsion method for satellite or spacecraft use, it is important to characterize the systems' lowest possible performance metrics, as well as nominal operation. Characterization of the lowest performance parameters provides valuable information on the smallest control torques available to the spacecraft during cold starts and initial pulsing mode operations, which can be used for fine attitude control. In order to gain an understanding of the smallest torques Pocket Rocket will impart during these transient periods, the 9 nozzle geometries from Table 2.1 will be simulated to represent initial thruster heating within the first 10 seconds of operation, after volumetric heating effects have reached thermal equilibrium, but before full wall heating effects have. Full thermal equilibrium with the thruster walls occurs within 100 seconds of operation [1]. Additionally, by simulating the 9 nozzle geometries from Table 2.1, a broad range of micro-nozzle flows can be characterized for Pocket Rocket, in order to determine if the addition of a nozzle can increase performance. This eliminates excessive laboratory time and costs associated with manufacturing and physically testing every single nozzle design.

Given the previously applied CFD boundary conditions in CFD-ACE+, as validated by experimental pressure ratio measurements in section 4.2 , confidence is realized for simulating the 9 nozzle geometries. However, several Pocket Rocket variables make the Cal Poly thruster used in this work unique to the original ANU developed thruster, whose estimated preliminary performance values are given in Table $1.2[1]$. These variables can be characterized by the physical design changes in the Cal Poly thruster for a slightly smaller plenum chamber, and through the use of copper tape as the powered RF electrode. Although the thruster used in this work made for a good testing candidate, given its ease of use and modularity with respect to the nozzle end-plate, these physical changes may introduce performance degradation. In 
order to characterize how each nozzle geometry will perform with Pocket Rocket in its original design and operating configuration, the CFD domain from section 4.1 is adapted to replicate the slightly larger plenum chamber from the original design [1]. Since the simulations will characterize Pocket Rocket performance from a cold start state, the housing components for heat transfer modeling are no longer necessary, and have been removed to save computation time. Within the first 10 seconds of operation, Pocket Rocket will only obtain full volumetric heating effects, as well as initial heating of the alumina discharge tube walls. In total, the adapted domain consists of mesh densities between 12971-13909 cells, with longer nozzles consisting of higher mesh densities. This range is slightly higher than the converged mesh cell density of 12882 cells used in the previously validated orifice fluid domain from section 4.2. An example domain can be seen in Figure 4.14, where the aluminum nozzle region replicated each of the 9 nozzle geometries.

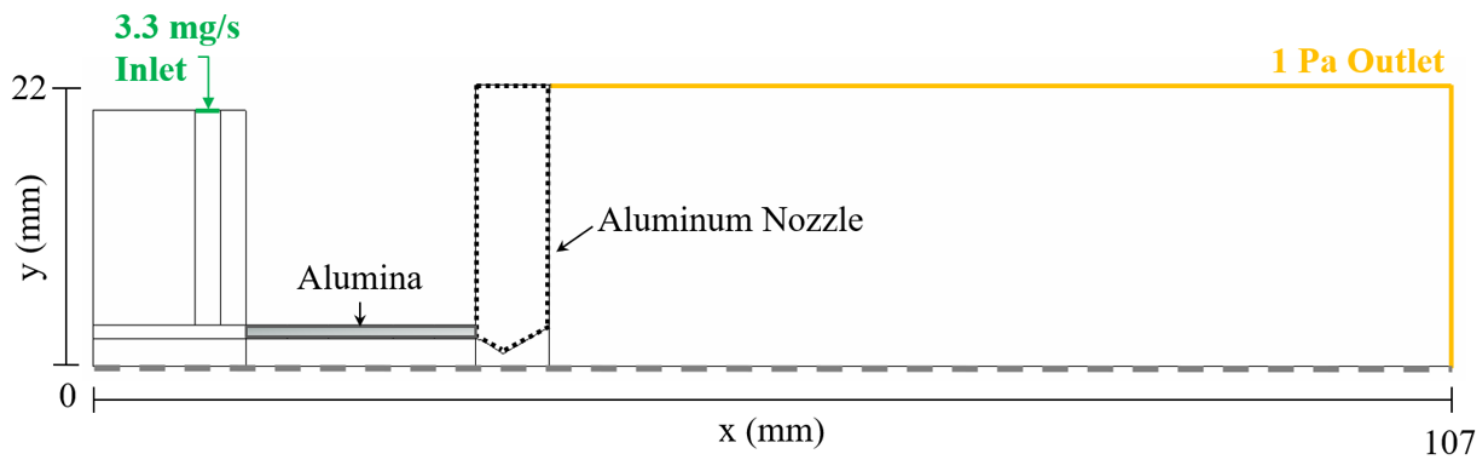

\section{Figure 4.14: Adapted CFD domain for the MN-1 through MN-9 nozzle geometry simulations.}

Each mesh in the CFD domain once again employs a larger cell size towards the upper downstream region of the fluid domain where the flow will not be studied. Cell density remains more refined in the regions of interest, such as along the inside of the alumina tube surface and throughout the nozzle. 
Table 4.2: Cold gas simulation performance results for mass flow rate $\dot{m}=3.3 \mathrm{mg} / \mathrm{s}$, ambient pressure $P_{a}=1 \mathrm{~Pa}$, plenum pressure $P_{p l}$, average exit exhaust velocity $v_{e x}$, thrust $F$, and specific impulse $I_{s p}$. Geometries vary with diverging half angle $\alpha$ and area ratio $\epsilon$.

\begin{tabular}{lllllll} 
Configuration & $\alpha$ & $\epsilon$ & $\begin{array}{l}P_{p l} \\
(\mathrm{~Pa})\end{array}$ & $\begin{array}{l}v_{\text {ex }} \\
(\mathrm{m} / \mathrm{s})\end{array}$ & $\begin{array}{l}\mathrm{F} \\
(\mathrm{mN})\end{array}$ & $\begin{array}{l}I_{s p} \\
(\mathrm{~s})\end{array}$ \\
\hline Orifice & 0 & 1 & 202.7 & 260 & 0.86 & 26.5 \\
MN-1 & 15 & 5 & 290 & 310 & 1.03 & 31.6 \\
MN-2 & 15 & 10 & 480 & 315 & 1.04 & 32.11 \\
MN-3 & 15 & 100 & 4345 & 330 & 1.09 & 33.64 \\
MN-4 & 30 & 5 & 283.7 & 317 & 1.05 & 32.31 \\
MN-5 & 30 & 10 & 468 & 325 & 1.08 & 33.13 \\
MN-6 & 30 & 100 & 4184 & 330 & 1.09 & 33.64 \\
MN-7 & 60 & 5 & 304.8 & 250 & 0.83 & 25.48 \\
MN-8 & 60 & 10 & 480 & 250 & 0.83 & 25.48 \\
MN-9 & 60 & 100 & 4194 & 275 & 0.91 & 28.03 \\
\hline
\end{tabular}

All simulations for the 9 nozzle geometries used the same boundary conditions outlined in section 4.1, except for the inlet and outlet. Since each nozzle design can significantly reduce the mass flow rate, and therefore thrust, as compared to the orifice configuration, simulations were ran at a constant $\dot{m}=3.3 \mathrm{mg} / \mathrm{s}$ at the inlet of the plenum chamber. Higher vacuum conditions of $1 \mathrm{~Pa}$ at the domain outlet were also used to simulate the thruster in a more typical flight-like environment, while still retaining the ability to converge under downstream vacuum conditions. Since performance parameters will be calculated from the thruster exit plane, a guarantee of exact plume modeling downstream, based on the continuation of the continuum flow regime, is of little concern. Cold gas simulations of the original orifice configuration and the 9 nozzle geometries were run first, with performance results shown in Table 
4.2. Exit velocity was taken to be the average over the exit plane of the nozzle and discharge tube flow, while thrust was estimated to be momentum thrust in Equation 1.1 .

From Table 4.2, it is clear that every nozzle design increased cold gas performance, except for the MN-7 through MN-9 geometries. These nozzles with half angles of $60^{\circ}$ experienced significant flow detachment with the steepest nozzle diverging contours. This can be seen in Figure 4.15 for the MN- 8 nozzle. For the $\alpha=60^{\circ}$ nozzles, flow detachment occurs just after the nozzle throat, thereby significantly reducing average exhaust velocity across the exit plane.

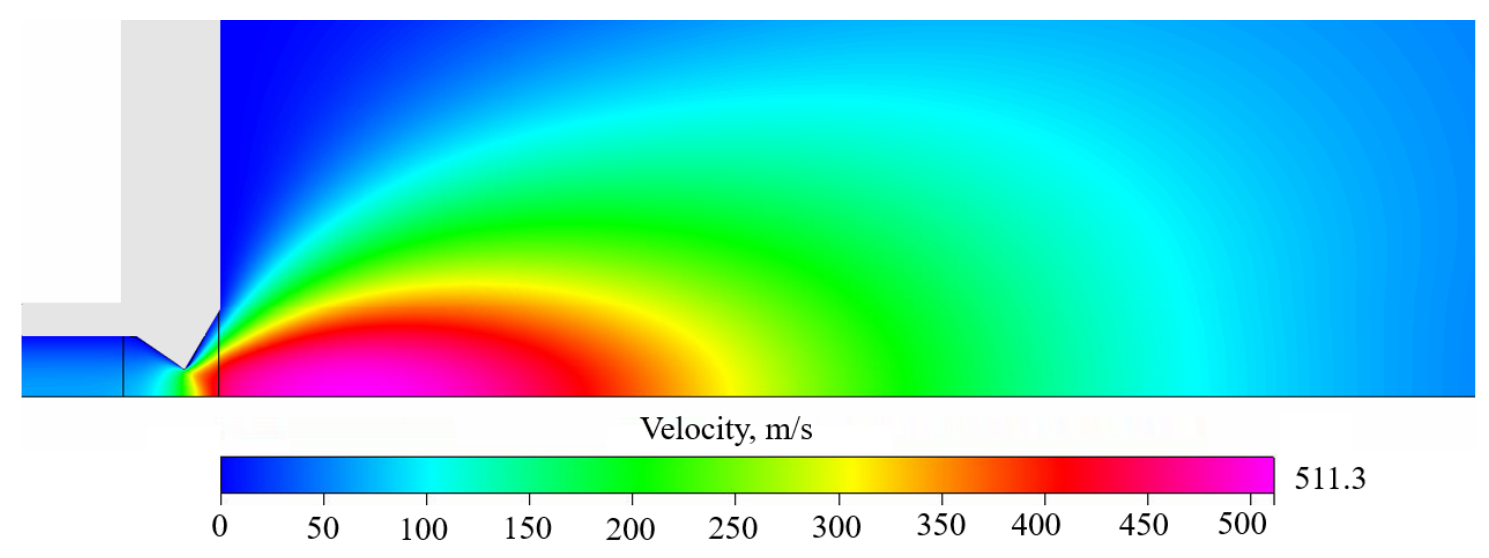

Figure 4.15: Flow detachment for the MN-8 nozzle.

Plasma heating was once again simulated in the steady state simulations by applying a constant volumetric power density of $5.8 \times 10^{6} \mathrm{~W} / \mathrm{m}^{3}$ to the thruster's discharge tube fluid volume. Fixed temperatures are applied to the gas discharge tube electrodes based on previous studies. Previously validated CFD results have indicated wall heating of up to $700 \mathrm{~K}$ on the powered electrode interface, while grounded electrodes reached temperatures of $400 \mathrm{~K}$ [1] during initial operation just have cold start. Expected neutral gas heating in Pocket Rocket has been found to be on the order of approximately $700 \mathrm{~K}$ after 10 seconds of operation. In order to simulate this initial heating effect, the alumina tube solid domain was given constant volumetric condi- 

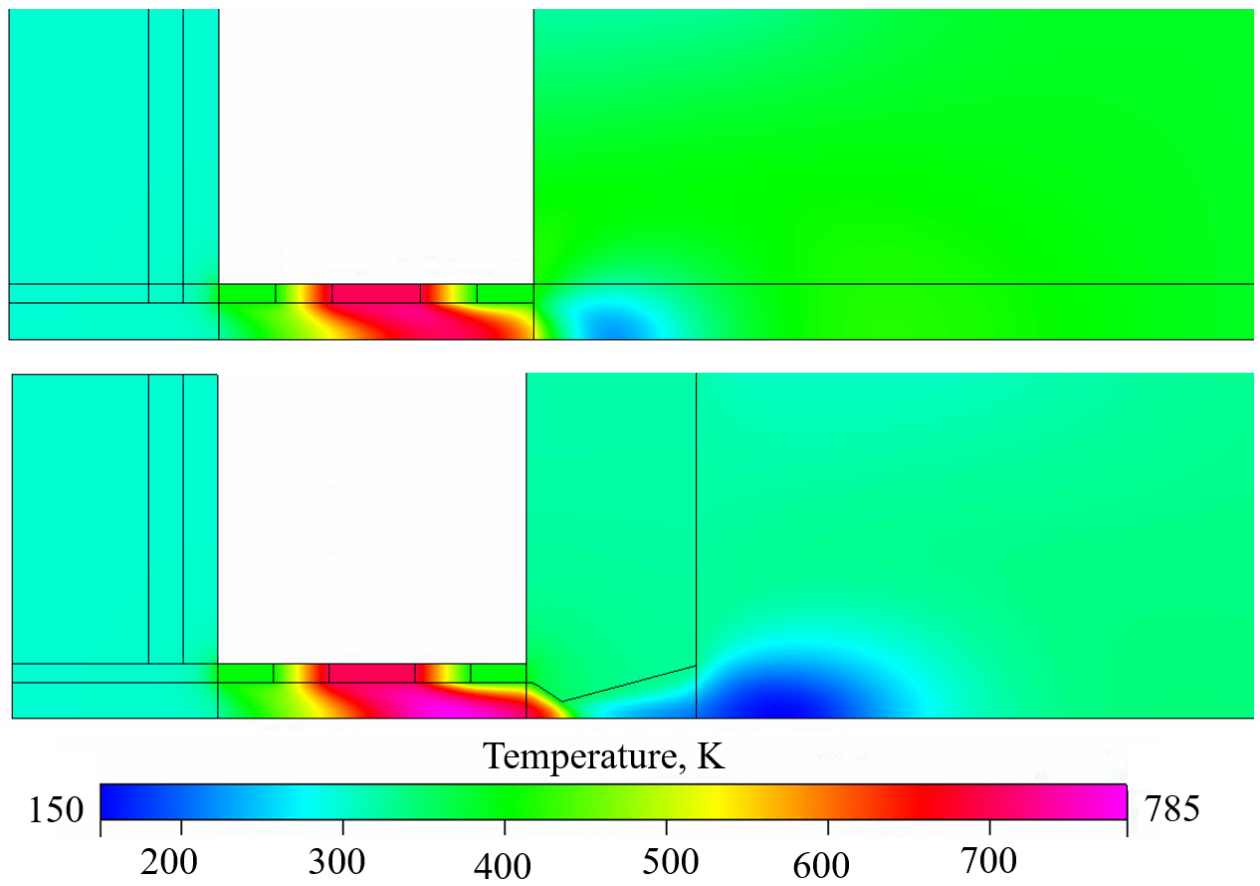

Figure 4.16: Simulated heating profile for (top) orifice thruster configuration and (bottom) MN-2 nozzle geometry.

tions of $700 \mathrm{~K}$ at the copper electrode interface, and $400 \mathrm{~K}$ at the grounded tube ends. An example of the simulated heating profile can be see in Figure 4.16, comparing the orifice configuration to the MN-2 nozzle geometry. Simulated temperatures of just over $700 \mathrm{~K}$ for the orifice configuration indicate good agreement with previous research [1].

The temperature ranges for neutral gas heating in the simulations ranged from 720-800 K. As Figure 4.16 shows, the orifice configuration provides the lowest neutral gas temperatures, with a peak of $722 \mathrm{~K}$ in the gas discharge tube. The MN-2 nozzle configuration once again provides better heat transfer by limiting gas expansion through the discharge tube. The nozzle configuration in Figure 4.16 also predicts a much lower global temperature at $150 \mathrm{~K}$, than that of the discharge tube orifice at 228 K. This is a strong indication that the nozzle has converted more thermal energy into kinetic exhaust energy by further reducing the downstream pressure and temperature $[8]$. 
A full table of CFD performance values from the 9 nozzle geometries, with the addition of the heat model, can be seen in Table 4.3. Once again, the MN-7 through MN-9 nozzles saw significant flow detachment. The top 2 performing nozzles were the MN-4 and MN-5 configurations. Although the difference in performance between these 2 nozzles is negligible, the MN-5 nozzle still demonstrated the highest increase in performance over the orifice configuration, with a thrust of $1.34 \mathrm{mN}$ and average exit velocity of $405 \mathrm{~m} / \mathrm{s}$. In comparison, the orifice configuration produced $1.26 \mathrm{mN}$ of thrust with an average exit velocity of $380 \mathrm{~m} / \mathrm{s}$.

Table 4.3: Simulated plasma heating performance results for mass flow rate $\dot{m}=3.3 \mathrm{mg} / \mathrm{s}$, ambient pressure $P_{a}=1 \mathrm{~Pa}$, plenum pressure $P_{p l}$, neutral gas temperature $T_{g}$, average exit exhaust velocity $v_{e x}$, thrust $F$, and specific impulse $I_{s p}$. Geometries vary with diverging half angle $\alpha$ and area ratio $\epsilon$.

\begin{tabular}{lccccccc} 
Configuration & $\alpha$ & $\epsilon$ & $\begin{array}{c}P_{p l} \\
(\mathrm{~Pa})\end{array}$ & $\begin{array}{c}T_{g} \\
(\mathrm{~K})\end{array}$ & $\begin{array}{c}v_{\text {ex }} \\
(\mathrm{m} / \mathrm{s})\end{array}$ & $\begin{array}{c}\mathrm{F} \\
(\mathrm{mN})\end{array}$ & $\begin{array}{c}I_{s p} \\
(\mathrm{~s})\end{array}$ \\
\hline Orifice & 0 & 1 & 353.3 & 722.6 & 380 & 1.26 & 38.74 \\
MN-1 & 15 & 5 & 434.3 & 763.1 & 375 & 1.24 & 38.23 \\
MN-2 & 15 & 10 & 656.4 & 784.2 & 376 & 1.25 & 38.33 \\
MN-3 & 15 & 100 & 4348 & 794.2 & 329 & 1.09 & 33.54 \\
MN-4 & 30 & 5 & 428.6 & 761.5 & 404 & 1.34 & 41.18 \\
MN-5 & 30 & 10 & 644.4 & 783.7 & 405 & 1.34 & 41.28 \\
MN-6 & 30 & 100 & 5375 & 793 & 405 & 1.34 & 41.29 \\
MN-7 & 60 & 5 & 455.2 & 767.8 & 322 & 1.07 & 32.82 \\
MN-8 & 60 & 10 & 661.5 & 784.3 & 311 & 1.03 & 31.7 \\
MN-9 & 60 & 100 & 4194 & 791.8 & 273 & 0.9 & 27.83 \\
\hline
\end{tabular}

The MN-6 nozzle produced similar increases in performance in compassion to MN-4 and MN-5 nozzles. However, the discharge tube and plenum pressures required upwards of $4000 \mathrm{~Pa}$ to maintain a constant mass flow rate of $3.3 \mathrm{mg} / \mathrm{s}$. This far exceeds 
the thruster's pressure distance limits for plasma breakdown. Given an applied 240 $\mathrm{V} R F$, the pressure-distance requirement for breakdown within the discharge tube is $p d=2.24-28.06$ Torr-mm. None of the nozzle geometries with $\epsilon=100$ meet the pd requirements, and will not allow for thruster operation at the nominal $3.3 \mathrm{mg} / \mathrm{s}$ mass flow rate. This can clearly be seen in the Paschen curve for argon gas in Figure 4.17, in which every nozzle pressure-distance value is plotted along the curve.

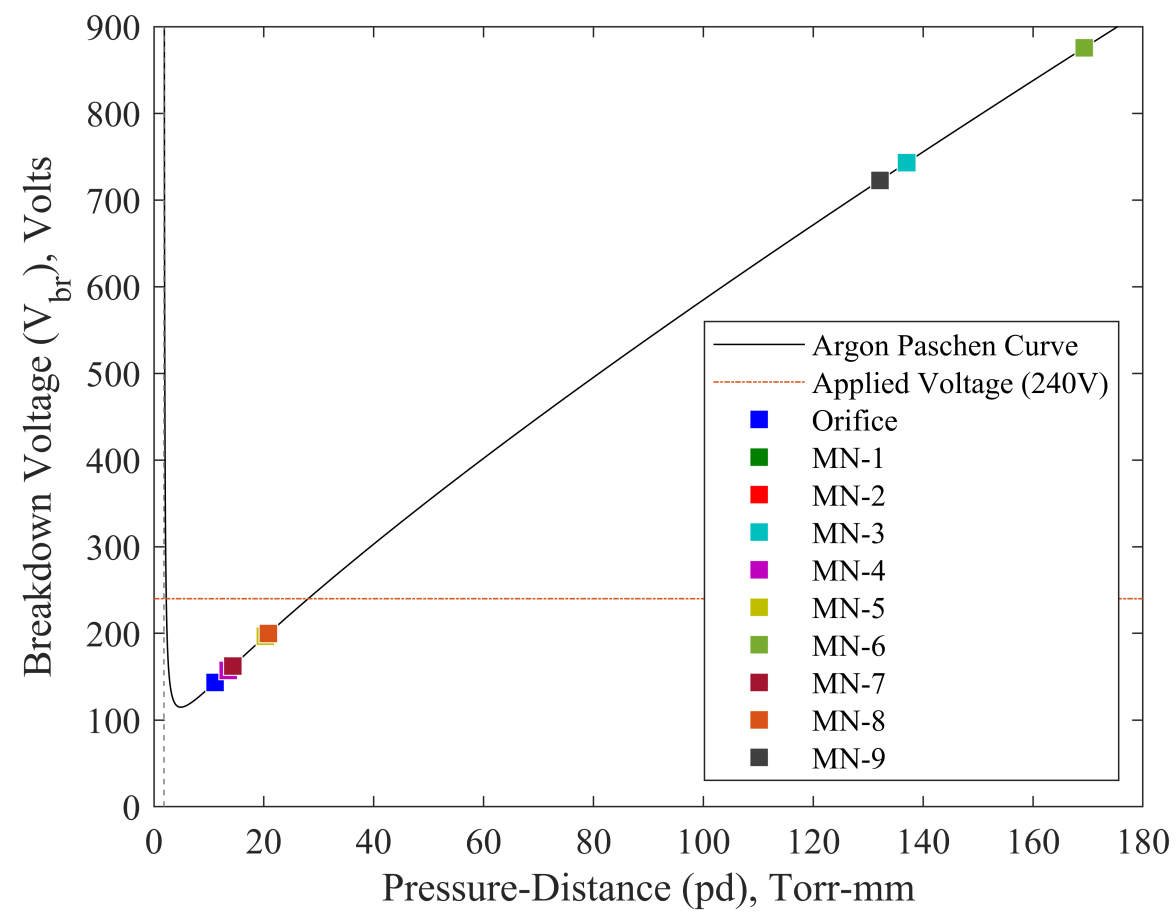

Figure 4.17: Simulation pressure-distance values along an argon Paschen curve.

The results in Table 4.3 suggest that the best preforming conical nozzle tested has a diverging half angle of $\alpha=30^{\circ}$ and a smaller area ratio around $\epsilon=5-10$. Even if the higher area ratio MN-6 nozzle did allow for plasma breakdown in the discharge tube, the performance increase was seen to be $<1 \%$ when compared to the MN-5 geometry.

A velocity profile comparison of the three nozzles with area ratios $\epsilon=10$ and half angles of $15^{\circ}, 30^{\circ}$, and $60^{\circ}$ can be seen in Figure 4.18. The results from Figure 
4.19 show the velocity at the exit plane radius for the same nozzles shown in Figure 4.18. These velocity profiles further suggest that the optimal diverging half-angle

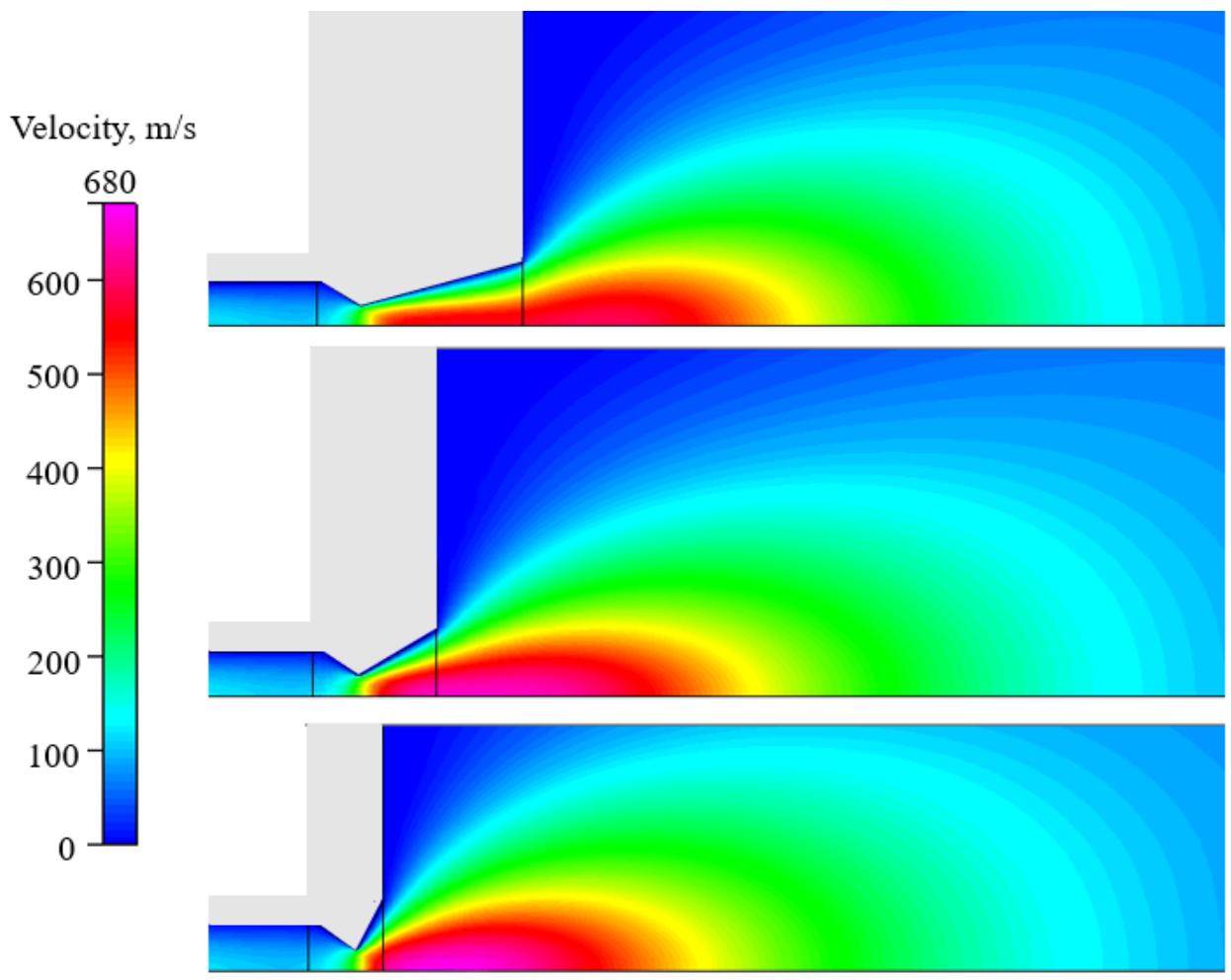

Figure 4.18: Simulated heating nozzle velocity profile comparisons for (top) MN-2 geometry, (middle) MN-5 geometry, and (bottom) MN-8 geometry $\left(\dot{m}=3.3 \mathrm{mg} / \mathrm{s}, P_{a}=1 \mathrm{~Pa}\right)$.

will be around $\alpha=30^{\circ}$. The top model in Figure 4.18 shows the MN-2 nozzle with $\alpha=15^{\circ}$, the middle model shows the MN-5 nozzle with $\alpha=30^{\circ}$, and the bottom model shows the MN-8 nozzle with $\alpha=60^{\circ}$. As can be seen in Figure 4.18 and Figure 4.19, the flow within the MN-2 nozzle shows a significantly larger boundary layer, in comparison, in the diverging section of the nozzle. This is in good agreement with previous research, which has suggested that the optimal micro-nozzle will require larger half angles in order to minimize nozzle cone length [11]. By minimizing the cone length of the nozzle, large viscous boundary layers can be minimized with a decrease in flow surface area. However, the MN-8 nozzle clearly shows that a further increase in $\alpha$ past $30^{\circ}$ can lead to significant flow detachment. 


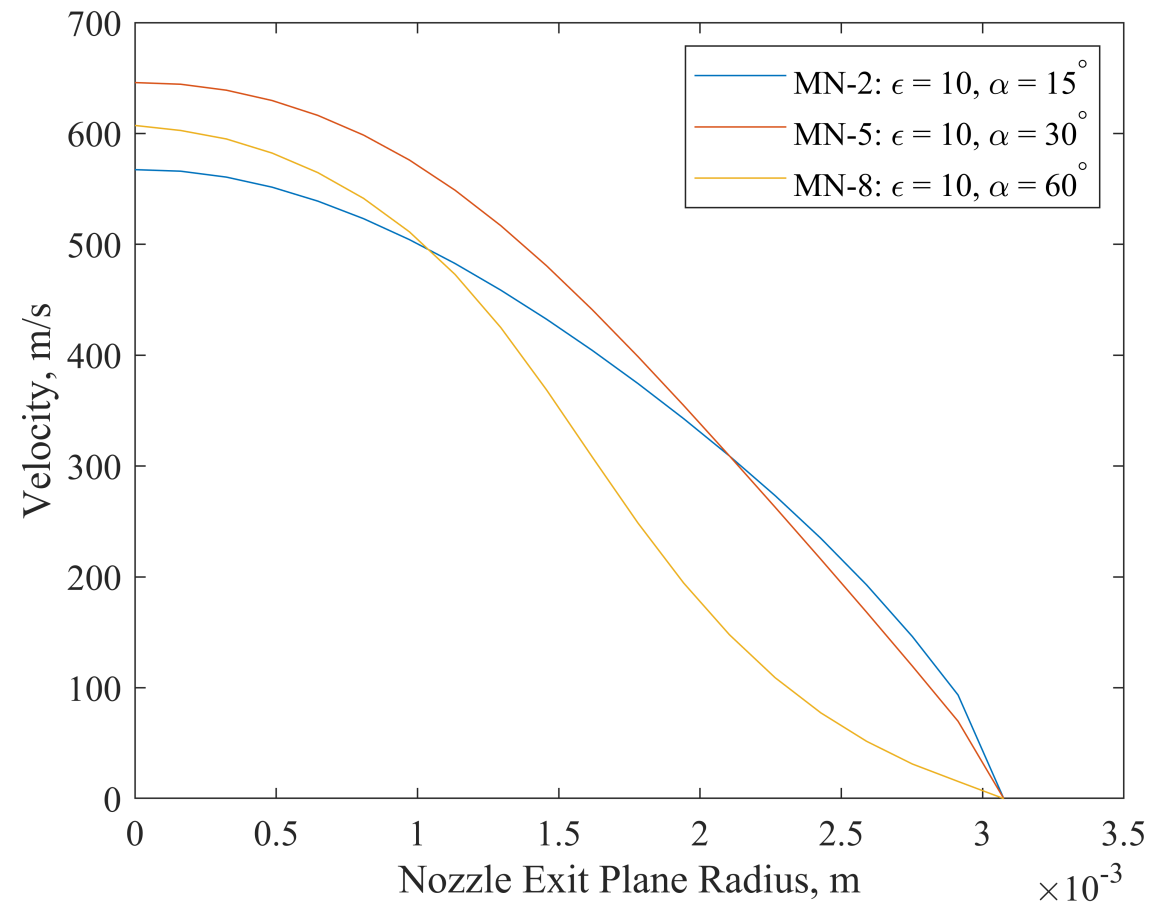

Figure 4.19: Simulated heating nozzle exit velocity plots for the MN-2, $\mathrm{MN}-5$, and MN-8 geometries $\left(\dot{m}=3.3 \mathrm{mg} / \mathrm{s}, P_{a}=1 \mathrm{~Pa}\right)$.

Overall, Table 4.3 shows lower specific impulse and thrust than the previously stated preliminary performance estimates for Pocket Rocket [1]. There are two main reasons for the decrease in stated performance. The CFD simulations used in this work represent the lowest range of neutral gas heating that occurs in Pocket Rocket from a cold start. Although this representation is useful for approximating the smallest control torque a satellite might use upon initial start up, the exit exhaust velocities are lower than what they would be otherwise, given nominal neutral gas temperatures (upwards of $1000 \mathrm{~K}$ ) after $100 \mathrm{~s}$ of operation [1]. In addition, the preliminary performance estimations assume a constant exit velocity across the orifice exit plane. This exit velocity is estimated by calculating the thermal particle velocity, given the measured neutral gas heating value of $1060 \mathrm{~K}$, and does not take into account boundary layer losses in the flow. This is most readily apparent from the global maximum velocity of approximately $700 \mathrm{~m} / \mathrm{s}$ for the simulated orifice configuration, seen in 
Figure 4.20. Although this simulated maximum velocity is in good agreement with the preliminary performance estimate of $v_{e x}=750 \mathrm{~m} / \mathrm{s}$ from Table 1.2 , the average velocity across the thruster exit plane ends up decreasing to $380 \mathrm{~m} / \mathrm{s}$. This causes a further reduction in thrust and specific impulse estimation. Despite these differences, full thermal equilibrium operation for Pocket Rocket will continue to provide an increase in performance past the values given in Table 4.3. This again leaves Pocket Rocket as a viable CubeSat thruster, with similar performance as other METs and RFETs $[15,13,14]$.

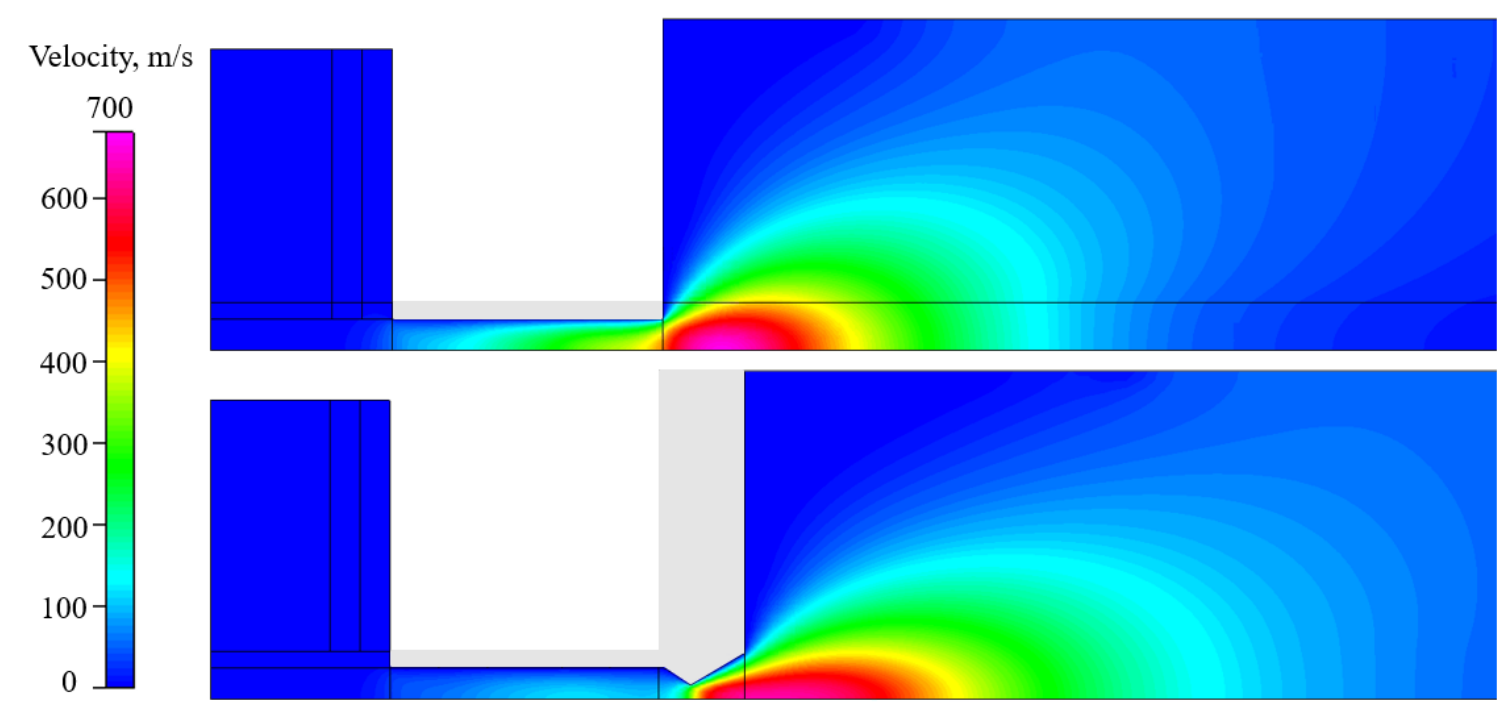

Figure 4.20: Simulated heating velocity profile comparisons for (top) orifice thruster geometry, and (bottom) MN-5 nozzle geometry $(\dot{m}=3.3$ $\left.\mathrm{mg} / \mathrm{s}, P_{a}=1 \mathrm{~Pa}\right)$.

With the MN-5 nozzle characterized as the highest performing nozzle in the range of the 9 conical geometries tested, further comparisons to the original orifice configuration can be made. Although the performance increase over the discharge tube orifice with the addition of the MN-5 nozzle is a modest $6 \%$, the nozzle does show promise as a valuable addition by increasing neutral gas heating up to $60 \mathrm{~K}$. By shifting the pressure gradient that extends through the entire discharge tube downstream to the nozzle's throat section, a higher pressure can be retained in the discharge tube, 
leading to an increase in effective propellant heating and performance.

Figure 4.20 compares the overall exhaust velocity plume of the orifice tube configuration and the MN-5 nozzle configuration. The shift in pressure gradient with the addition of the nozzle is once again clear as the flow in the orifice tube is seen to expand and accelerate in the discharge tube, while the nozzle configuration shifts the pressure gradient to the confines of the nozzle throat. This shift in flow may also help in plasma confinement. Previous research has indicated that a micro-nozzle sculpted in the discharge tube itself, near the RF electrode, may increase plasma confinement in Pocket Rocket due to plasma sheaths that develop in the throat of the nozzle [25]. Future research may include determining where the optimal nozzle location is that will contribute to an increase in plasma confinement due to the development of plasma sheaths, as well as the shift in pressure gradient in the nozzle's throat.

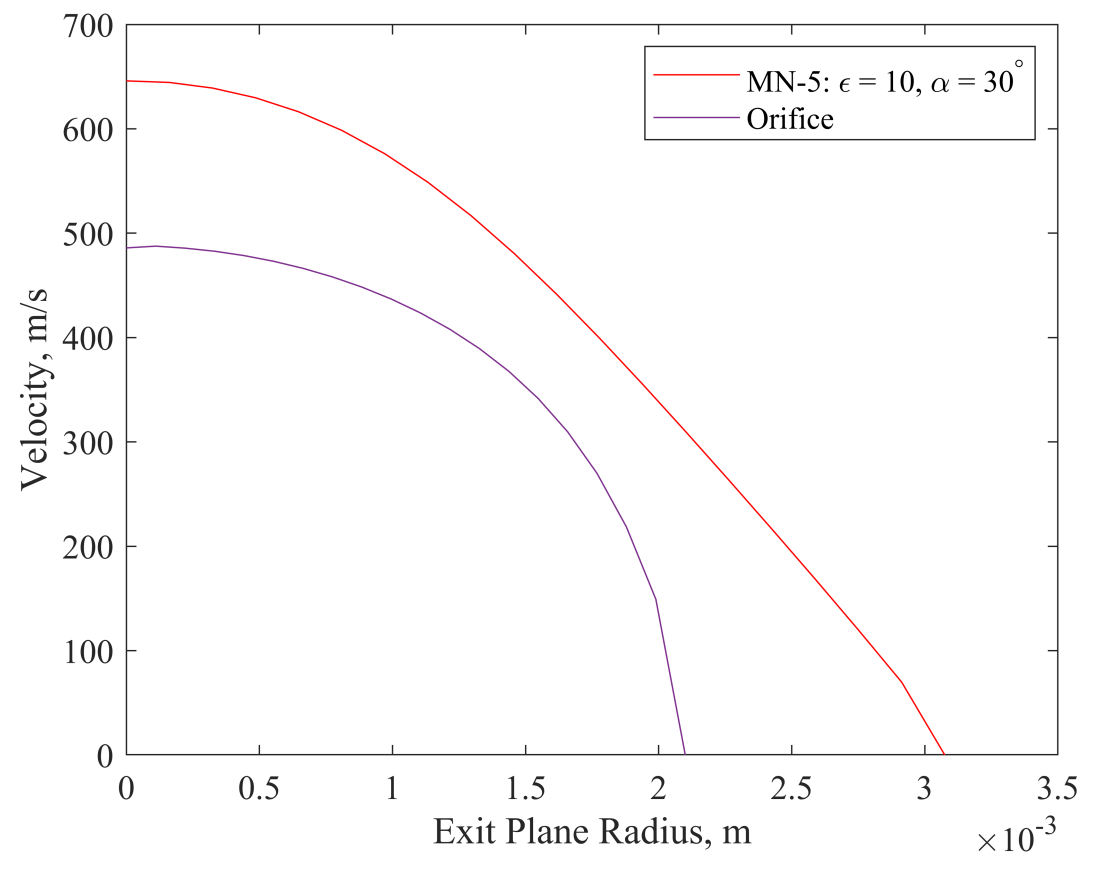

Figure 4.21: Simulated heating exit velocity plot comparison for the orifice and MN-5 nozzle geometries $\left(\dot{m}=3.3 \mathrm{mg} / \mathrm{s}, P_{a}=1 \mathrm{~Pa}\right)$. 
The exhaust plume in Figure 4.20 also clearly shows an increase in axial thrust with the addition of a nozzle, as the flow in the orifice configuration expands into the the y-axis more immediately, thereby losing thrust from potential flow that could otherwise be diverted along the $\mathrm{x}$-axis. The increase in velocity at the exit plane of the nozzle can also be seen in Figure 4.21. From the exit plane radius of the MN-5 nozzle, peak velocity is shown to increase by $159 \mathrm{~m} / \mathrm{s}$ in the center of the flow, over the orifice geometry.

Perhaps the largest value to adding a conical nozzle to the end of the discharge tube for Pocket Rocket is the increase in performance as a cold gas thruster. Although the performance increase from the orifice to MN-5 nozzle configuration during plasma operation is only $6 \%$, the performance increase with the addition of the nozzle as a cold gas thruster is up to $25 \%$. This is a significant increase that would be highly beneficial in a contingency scenario where the thruster lost power and could no longer generate a plasma. The addition of a nozzle would also be beneficial for orbital maneuvers and station keeping in times of low power, such as during orbital eclipse periods. One reason why the same $25 \%$ nozzle performance gain was not seen during plasma operation is that the original discharge tube orifice configuration may act inherently like a plug nozzle itself, during plasma operation. An additional increase in exhaust acceleration may be provided in the orifice discharge tube as the plasma bulk creates a plug in the middle of the flow, thereby accelerating flow around this plug to higher velocities. Future work should investigate this possible phenomena through additional transient plasma CFD studies. It is also very likely that viscous losses simply increase during plasma operation. Although thermal velocity significantly increases when the temperature of a propellant gas increases, viscosity can also increase and inhibit performance through boundary layer development. 
Chapter 5

CONCLUSION

This thesis presented an experimental and computational study aimed at discovering if the addition of a micro-nozzle, placed at the end of the gas discharge tube in an electrothermal plasma thruster, would increase performance. Characterization of micro-nozzle flows within the Pocket Rocket thruster have been performed. The simulations presented in this work have been validated by testing the Pocket Rocket thruster with a conical $\alpha=30^{\circ}, \epsilon=10$ nozzle, referred to as MN-5. This nozzle was selected and manufactured for test as it represents the middle of the range of selected nozzle geometries that were designed to both bound a large range of thrust coefficient values, and minimize viscous boundary layer losses within the flow. After measuring static and total pressures at various locations along the length of the thruster's exhaust plume, the experimental pressure ratio $\frac{P_{s}}{P_{0}}$ was able to match the CFD results within a $3 \sigma$ error. Error in the experimental results could be reduced by using convectron gauges that are specifically calibrated for argon gas use, since a slightly ambiguous convectron gas correction factor was required for testing in this work. During testing of the thruster configuration with the MN-5 nozzle, standing shock diamonds were observed to appear in the flow. The oblique shock structures surrounding these shock diamonds were detected in the flow with a pitot static tube and convectron gauges. It is possible that the experimental results over-predicted the pressure ratio across the tested nozzle's standing oblique shocks due to shock-shock interactions with the pitot tube's own bow shock, created within the supersonic flow.

Overall, this research suggests that a nozzle located at the end of the gas discharge tube may be highly beneficial to the Pocket Rocket thruster during periods of low power, or during contingency scenarios as cold gas thruster. CFD simulations show 
that the addition of the MN-5 nozzle can improve performance over the original orifice configuration by $25 \%$ with cold gas operation. A more modest increase in performance of $6 \%$ was seen with the use of the MN-5 nozzle during plasma operation. One reason for this smaller performance increase is that the plasma may already act as a form of plug-nozzle within the gas discharge tube. This could result in a smaller additional increase in performance with the use of a physical nozzle. Viscous losses can also be attributed to a smaller increase in overall nozzle plasma performance as viscosity increases within a heated gas.

Each nozzle configuration simulated also showed an increase in neutral gas heating within Pocket Rocket's gas discharge tube. When the orifice discharge tube is used with the thruster, the pressure gradient acts along the entire length of the tube, thereby expanding and cooling the propellant gas through the plasma bulk region. However, when a nozzle is placed at the end of the gas discharge tube, the pressure gradient is reduced to the vicinity of the nozzle's throat. This leads to an increase in pressure in the gas discharge tube where the plasma is generated, and leads to an increase in effective propellant heating. Previous CFD studies on the use of a nozzle within the gas discharge tube have also found that plasma confinement may be increased with the use of a nozzle. However, this plasma confinement increase may be attributed to plasma sheaths that form around the throat of the nozzle near the RF electrode [25]. Future work should include optimizing the location of the nozzle, such that the highest effective propellant heating and plasma confinement can be achieved. It is possible that the shift in pressure gradient by placing the nozzle at the end of the discharge tube could also lead to more effective plasma confinement, as the propellant is no longer being accelerated as much through the plasma bulk.

The outcomes of this work include the demonstration of a low cost method of characterizing the exhaust flow of an electrothermal plasma thruster with the use of a pitot static tube and two convectron gauges. While other means to characterize a 
thruster with propulsive force on the order of $1 \mathrm{mN}$ exist, the selected instrumentation provides accurate flow characterization at a significantly lower cost. This work as also demonstrated that the best performance enchaining micro-nozzle simulated had an $\alpha=30^{\circ}$ and $\epsilon=10$ out of the large range of half angles and area ratios (from $\alpha=15^{\circ}-60^{\circ}$ and $\epsilon=5-100$ ). This characterization of a conical micro-nozzle geometry may also be applied to other micro-thrusters under development, and help to provide an increase in propulsive functionality for the SmallSat class of satellites and spacecraft. Recommended future work includes finding the exact nozzle half angle and area ratio that would best suit the thruster on a mission to mission basis, based on the constraints on weight and performance of the propulsion system. Future work may also include modeling other nozzle shapes and contours for comparison to the conical geometry. 


\section{BIBLIOGRAPHY}

[1] A. Greig. Pocket rocket: An electrothermal plasma micro-thruster. A thesis submitted for the degree of Doctor of Philosophy of The Australian National University, pages 1-130, 2015.

[2] MKS Pressure and Vacuum Measurement Solutions. Series 275 instruction manual granville-phillips convectron vacuum gauge bridge amp pc board with analog output signal, 2016.

[3] Inc. InstruTech. Ionization vacuum gauge controller with dual convection b-rax 3000, 2008.

[4] et. al H. Heidt. Cubesat: A new generation of picosatellite for education and industry low-cost space experimentation. AIAA Small Satellite Conference, pages $1-18,2000$.

[5] A. Dutta A. Tummala. An overview of cube-satellite propulsion technologies and trends. Aerospace, 4(4):1-58, 2017.

[6] et. al J. Moerel. Development of micro propulsion system technologies for minisatellite in the netherlands. (November 2015):1-10, 2008.

[7] T. Werne J. Schoolcraft, A. Klesh. Marco: interplanetary mission development on a cubesat scale. In 14 th International Conference on Space Operations, page 2491, 2016.

[8] O. Biblarz G. Sutton. Rocket Propulsion Elements. John Wiely \& Sons, Inc., 8 edition, 2010.

[9] D. Huang D. Huzel. Modern Engineering for Design of Liquid-Propellant Rocket Engines, volume 147. AIAA, 1992. 
[10] M. Osborn L. Williams. Performance impacts of geometry and operating conditions on a low reynolds number micro-nozzle flow. IEPC, 120:1-12, 2017.

[11] F. La Torre. Gas Flow in Miniaturized Nozzles for Micro-Thrusters. 2011.

[12] I. Katz D. Goebel. Fundamentals of Electric Propulsion: Ion and Hall Thrusters. JPL SPACE SCIENCE AND TECHNOLOGY SERIES, 2008.

[13] Takeshi Takahashi, Yoshinori Takao, Koji Eriguchi, and Kouichi Ono. Numerical and experimental study of microwave-excited microplasma and micronozzle flow for a microplasma thruster. Physics of plasmas, 16(8):083505, 2009.

[14] Yoshinori Takao, Koji Eriguchi, and Kouichi Ono. A miniature electrothermal thruster using microwave-excited microplasmas: Thrust measurement and its comparison with numerical analysis. Journal of Applied Physics, 101(12):123307, 2007.

[15] I. Hrbud W. Stein, A. Alexeenko. Performance modeling of a coaxial radio-frequency gas-discharge microthruster. Journal of Propulsion and Power, 24(5):1007-1017, 2008.

[16] M. Patterson G. Ganapathi J. Brophy H. Richard G. Schmidt, D. Jacobson. Electric propulsion research and development at nasa. 2018.

[17] R. Hawkins R. Boswell A. Greig, C. Charles. Direct measurement of neutral gas heating in a radio-frequency electrothermal plasma micro-thruster. Applied Physics Letters, 103(7):10-14, 2013.

[18] R. Boswell C. Charles. Measurement and modelling of a radiofrequency micro-thruster. Plasma Sources Science and Technology, 21(2):17-21, 2012. 
[19] A review of plasma thruster work at the australian national university. In Presented at Joint Conference of 30th International Symposium on Space Technology and Science, pages 3-4, 2015.

[20] M. Gokowski U. Inan. Principles of Plasma Physics for Engineers and Scientists. Cambridge University Press, 2010.

[21] N. Braithwaite P. Chabert. Physics of Radio-Frequency Plasmas. Cambridge University Press, 2011.

[22] A. Lichtenberg M. Lieberman. Principles of plasma discharges and materials processing. John Wiley \& Sons, 2005.

[23] R. Boswell A. Greig, C. Charles. Simulation of main plasma parameters of a cylindrical asymmetric capacitively coupled plasma micro-thruster using computational fluid dynamics. Frontiers in Physics, 2(January), 2015.

[24] H. King. Electrode geometry effects in an electrothermal plasma microthruster. A Thesis presented to the Faculty of California Polytechnic State University, San Luis Obispo In, pages 1-52, 2018.

[25] R. Boswell T. Ho, C. Charles. Performance modelling of plasma microthruster nozzles in vacuum. Journal of Applied Physics, 123(17), 2018.

[26] R. Boswell T. Ho, C. Christine. A comprehensive cold gas performance study of the pocket rocket radiofrequency electrothermal microthruster. Frontiers in Physics, 4:55, 2017.

[27] A. Rivera L. Arrington, B. Reed. A performance comparison of two small rocket nozzles. In 32nd Joint Propulsion Conference and Exhibit, page 2582, 1996.

[28] A.H Silver T.J. Marcisz C.K. Murch, J. Broadwell. Low-thrust nozzle performance. AIAA 6th Aerospace Sciences Meeting, (68), 1968. 
[29] L. Saltz S. Grisnik, T. Smith. Experimental study of low reynolds number nozzles. IEPC, 87-0992:1-1, 1987.

[30] et. al A. Hunt. Guide to the Measurement of Force. Institute of Measurement and Control, 1998.

[31] H. Bengtson. Fluid velocity measurement using a pitot tube (pitot static tube). Hydraulics in Civil Engineering, 2010.

[32] S. Waterbury C. Powers. Outgassing data for selecting spacecraft materials, 2018.

[33] J. Liu K. Kailasanath D. Munday, E. Gutmark. Flow structure and acoustics of supersonic jets from conical convergent-divergent nozzles. Physics of Fluids, 23(11):116102, 2011.

[34] C. Zhao J. Xu. Two-dimensional numerical simulations of shock waves in micro convergent-divergent nozzles. International Journal of Heat and Mass Transfer, 50(11-12):2434-2438, 2007.

[35] S. Collicott D. Valentine E.L. Houghton, P.W. Carpenter. Chapter 6 compressible flow. In E.L. Houghton, P.W. Carpenter, Steven H. Collicott, and Daniel T. Valentine, editors, Aerodynamics for Engineering Students (Sixth Edition), pages 349 - 425. Butterworth-Heinemann, Boston, sixth edition edition, 2013.

[36] TA. Korotayeva IS. Tsyryulnikov SG. Mironov, VM. Aniskin. Effect of pitot-tube diameter on the measurements in an axisymmetric micro-jets. In AIP Conference Proceedings, volume 2027, page 030054. AIP Publishing, 2018.

[37] M. Groenendijk M. Elwenspoek M. C. Louwerse, H. V. Jansen. Nozzle 
fabrication for micropropulsion of a microsatellite. Journal of Micromechanics and Microengineering, 19(4), 2009.

[38] B. Chanetz J. Délery Jean F. Grasso, C. Purpura. Type iii and type iv shock/shock interferences: theoretical and experimental aspects. Aerospace Science and technology, 7(2):93-106, 2003. 


\section{APPENDICES}

Appendix A

\section{CONVECTRON CALIBRATION}

Supplemental convectron gauge calibration tools for argon gas correction.

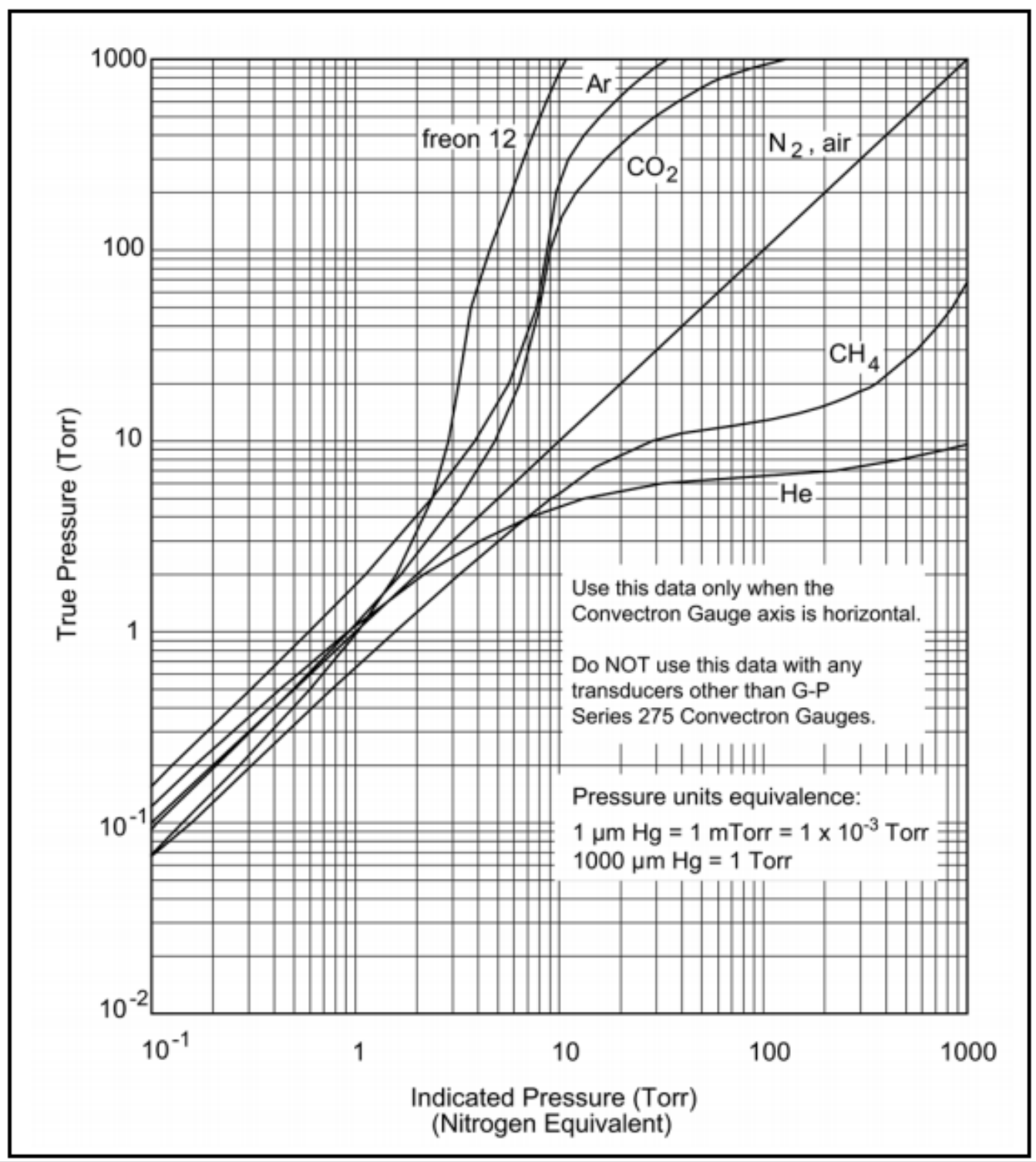

Figure A.1: MKS Gas Calibration Curve for Series 275 Convectron Gauge $[2]$. 
Table 3 - Displayed Pressure Readings vs Pressure, for Selected Gases for Convection Gauges

\begin{tabular}{|c|c|c|c|c|c|c|c|c|c|c|c|}
\hline True Pressure & N2 & $\mathrm{Ar}$ & $\mathrm{He}$ & $\mathrm{O} 2$ & $\mathrm{CO} 2$ & $\mathrm{Kr}$ & Freon12 & Freon 22 & D2 & $\mathrm{Ne}$ & $\mathrm{CH} 4$ \\
\hline $\begin{array}{|ll|}1.00 \mathrm{E}-4 & \text { Torr } \\
\end{array}$ & $1.00 \mathrm{E}-4$ & $1.00 \mathrm{E}-4$ & $1.00 \mathrm{E}-4$ & $1.00 \mathrm{E}-4$ & $1.00 \mathrm{E}-4$ & $1.00 \mathrm{E}-4$ & $1.00 \mathrm{E}-4$ & $1.00 \mathrm{E}-4$ & $1.00 \mathrm{E}-4$ & $1.00 \mathrm{E}-4$ & $1.00 \mathrm{E}-4$ \\
\hline $\begin{array}{|ll|}5.00 \mathrm{E}-4 & \text { Torr } \\
\end{array}$ & $5.00 E-4$ & $5.00 \mathrm{E}-4$ & $5.00 \mathrm{E}-4$ & $5.00 \mathrm{E}-4$ & $5.00 \mathrm{E}-4$ & $3.00 \mathrm{E}-4$ & $5.00 \mathrm{E}-4$ & $5.00 \mathrm{E}-4$ & $5.00 \mathrm{E}-4$ & $5.00 \mathrm{E}-4$ & $5.00 \mathrm{E}-4$ \\
\hline $\begin{array}{|ll|}2.00 E-3 & \text { Torr } \\
\end{array}$ & $2.00 E-3$ & $1.40 \mathrm{E}-3$ & $1.60 \mathrm{E}-3$ & $2.00 \mathrm{E}-3$ & $2.30 \mathrm{E}-3$ & $1.00 \mathrm{E}-3$ & $3.10 \mathrm{E}-3$ & $3.10 \mathrm{E}-3$ & $2.40 \mathrm{E}-3$ & $1.50 \mathrm{E}-3$ & $3.30 \mathrm{E}-3$ \\
\hline $\begin{array}{|ll|}5.00 \mathrm{E}-3 & \text { Torr } \\
\end{array}$ & $5.00 \mathrm{E}-3$ & $3.30 \mathrm{E}-3$ & $4.00 \mathrm{E}-3$ & $5.00 \mathrm{E}-3$ & $4.40 \mathrm{E}-3$ & $2.30 \mathrm{E}-3$ & $7.60 \mathrm{E}-3$ & $7.00 \mathrm{E}-3$ & $6.00 \mathrm{E}-3$ & $3.50 \mathrm{E}-3$ & $7.70 \mathrm{E}-3$ \\
\hline $\begin{array}{|ll|}1.00 \mathrm{E}-2 & \text { Torr } \\
\end{array}$ & $1.00 \mathrm{E}-2$ & $6.60 \mathrm{E}-3$ & $8.10 \mathrm{E}-3$ & $9.70 \mathrm{E}-3$ & 1.10E-2 & $4.80 \mathrm{E}-3$ & $1.47 \mathrm{E}-2$ & $1.35 \mathrm{E}-2$ & $1.21 \mathrm{E}-2$ & $7.10 \mathrm{E}-3$ & $1.53 E-2$ \\
\hline $\begin{array}{|ll|}5.00 \mathrm{E}-2 & \text { Torr } \\
\end{array}$ & $5.00 \mathrm{E}-2$ & $3.24 \mathrm{E}-2$ & $4.05 \mathrm{E}-2$ & $4.92 \mathrm{E}-2$ & $5.49 \mathrm{E}-2$ & $2.35 \mathrm{E}-2$ & $7.25 \mathrm{E}-2$ & $6.90 \mathrm{E}-2$ & $6.00 \mathrm{E}-2$ & $3.48 \mathrm{E}-2$ & $7.72 \mathrm{E}-2$ \\
\hline $\begin{array}{|ll|}1.00 \mathrm{E}-1 & \text { Torr } \\
\end{array}$ & $1.00 \mathrm{E}-1$ & $6.43 \mathrm{E}-2$ & $8.20 \mathrm{E}-2$ & $9.72 \mathrm{E}-2$ & $1.07 \mathrm{E}-1$ & $4.68 \mathrm{E}-2$ & $1.43 E-1$ & $1.36 \mathrm{E}-1$ & $1.21 \mathrm{E}-1$ & $7.00 \mathrm{E}-2$ & $1.59 \mathrm{E}-1$ \\
\hline $\begin{array}{|ll|}2.00 \mathrm{E}-1 & \text { Torr } \\
\end{array}$ & $2.00 \mathrm{E}-1$ & $1.26 \mathrm{E}-1$ & $1.65 \mathrm{E}-1$ & $1.94 \mathrm{E}-1$ & $2.10 \mathrm{E}-1$ & $9.11 \mathrm{E}-2$ & $2.75 \mathrm{E}-1$ & $2.62 \mathrm{E}-1$ & $2.50 \mathrm{E}-1$ & $1.41 \mathrm{E}-1$ & $3.15 \mathrm{E}-1$ \\
\hline 5.00E-1 Torr & $5.00 E-1$ & $3.12 \mathrm{E}-1$ & 4.35E-1 & $4.86 \mathrm{E}-1$ & $4.89 \mathrm{E}-1$ & $2.17 \mathrm{E}-1$ & $6.11 \mathrm{E}-1$ & $5.94 \mathrm{E}-1$ & $6.87 \mathrm{E}-1$ & $3.59 \mathrm{E}-1$ & $7.81 \mathrm{E}-1$ \\
\hline $1.00 \mathrm{E}+0$ Torr & $1.00 \mathrm{E}+0$ & $6.00 \mathrm{E}-1$ & $9.40 E-1$ & $9.70 \mathrm{E}-1$ & $9.50 \mathrm{E}-1$ & $4.00 \mathrm{E}-1$ & $1.05 E+0$ & $1.04 E+0$ & $1.55 E+0$ & $7.45 \mathrm{E}-1$ & $1.60 \mathrm{E}+0$ \\
\hline $5.00 \mathrm{E}+1$ Torr & $5.00 \mathrm{E}+1$ & $7.85 \mathrm{E}+0$ & OP & $7.76 \mathrm{E}+1$ & $8.22 \mathrm{E}+0$ & $2.57 E+0$ & $3.79 E+0$ & $4.14 E+0$ & OP & OP & $8.42 \mathrm{E}+2$ \\
\hline $1.00 \mathrm{E}+2$ Torr & $1.00 \mathrm{E}+2$ & $8.83 \mathrm{E}+0$ & OP & $2.09 E+2$ & $9.25 \mathrm{E}+0$ & $2.74 E+0$ & $4.68 \mathrm{E}+0$ & $4.91 E+0$ & OP & OP & OP \\
\hline $2.00 \mathrm{E}+2$ Torr & $2.00 \mathrm{E}+2$ & $9.79 \mathrm{E}+0$ & OP & $2.95 E+2$ & $1.23 E+1$ & $3.32 E+0$ & $5.99 E+0$ & $6.42 E+0$ & OP & OP & OP \\
\hline $3.00 \mathrm{E}+2$ Torr & $3.00 \mathrm{E}+2$ & $1.13 \mathrm{E}+1$ & OP & $3.80 E+2$ & $1.69 \mathrm{E}+1$ & $3.59 E+0$ & $6.89 E+0$ & $7.52 \mathrm{E}+0$ & OP & OP & OP \\
\hline $4.00 \mathrm{E}+2$ Torr & $4.00 \mathrm{E}+2$ & $1.35 \mathrm{E}+1$ & OP & $4.85 E+2$ & $2.24 \mathrm{E}+1$ & $3.94 E+0$ & $7.63 E+0$ & $8.42 E+0$ & OP & OP & OP \\
\hline \begin{tabular}{|l|l|}
$5.00 \mathrm{E}+2$ Torr \\
\end{tabular} & $5.00 \mathrm{E}+2$ & $1.61 \mathrm{E}+1$ & $\mathrm{OP}$ & $6.04 \mathrm{E}+2$ & $2.87 \mathrm{E}+1$ & $4.21 \mathrm{E}+0$ & $8.28 \mathrm{E}+0$ & $9.21 \mathrm{E}+0$ & OP & OP & OP \\
\hline $6.00 \mathrm{E}+2$ Torr & $6.00 \mathrm{E}+2$ & $1.88 \mathrm{E}+1$ & OP & $7.30 \mathrm{E}+2$ & $3.64 \mathrm{E}+1$ & $4.44 E+0$ & $8.86 \mathrm{E}+0$ & $9.95 E+0$ & OP & OP & OP \\
\hline $7.00 \mathrm{E}+2$ Torr & $7.00 \mathrm{E}+2$ & $2.18 \mathrm{E}+1$ & $\mathrm{OP}$ & $8.59 E+2$ & $4.61 \mathrm{E}+1$ & $4.65 E+0$ & $9.42 E+0$ & $1.07 \mathrm{E}+1$ & OP & OP & OP \\
\hline $7.60 \mathrm{E}+2$ Torr & $7.60 \mathrm{E}+2$ & $2.37 \mathrm{E}+1$ & OP & $9.41 \mathrm{E}+2$ & $5.39 \mathrm{E}+1$ & $4.75 E+0$ & $9.76 E+0$ & $1.11 \mathrm{E}+1$ & OP & OP & OP \\
\hline $8.00 \mathrm{E}+2$ Torr & $8.00 \mathrm{E}+2$ & $2.51 \mathrm{E}+1$ & OP & $9.97 E+2$ & $5.94 E+1$ & $4.84 E+0$ & $9.95 E+0$ & $1.14 E+1$ & OP & OP & OP \\
\hline $9.00 \mathrm{E}+2$ Torr & $9.00 \mathrm{E}+2$ & $2.8 \mathrm{SE}+1$ & $O P$ & OP & $7.95 \mathrm{E}+1$ & $4.99 \mathrm{E}+0$ & 1.05E +1 & $1.20 \mathrm{E}+1$ & OP & OP & OP \\
\hline
\end{tabular}

Figure A.2: InstruTech, Inc. CVG-101 Convection Gauge Calibration Table [3]. 